$$
\begin{aligned}
& \text { Reigived by OSTI } \\
& \text { OCT } 091990
\end{aligned}
$$

A Reciprocal space Approach for Locating symmetry Elements in Patterson Superposition Maps

by

Hendrixson, Tom

PHD Thesis submitted to Iowa state University

Ames Laboratory, U.S. DOE

Iowa State University

Ames, Iowa 50011

Date Transmitted: September 21, 1990

PREPARED FOR THE U.S. DEPAFTMENT OF ENERGY

UNDER CONTRACT NO. W-7405-Eng-82. 


\title{
A reciprocal space approach for locating symmetry elements in Patterson superposition maps
}

\author{
by
}

Thomas L. Hendrixson

\author{
An Abstract of \\ A Dissertation submitted to the \\ Graduate Faculty in partial fulfillment of the \\ Requirements for the Degree of \\ DOCTOR OF PHILOSOPHY
}

Approved:

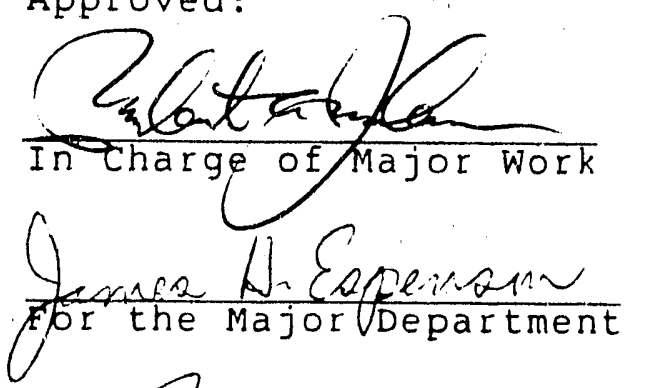

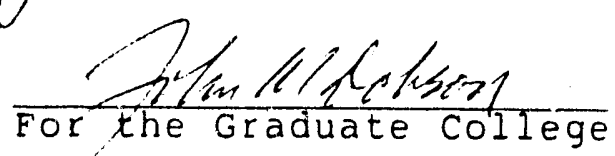

Iowa state University

Ames, Iowa

1989 


\begin{abstract}
A reciprocal space approach for locating symmetry elements in patterson superposition mape
\end{abstract}

Thomas L. Hendrixson

\author{
Under the supervision of R. A. Jacobson \\ From the Department of Chamistry \\ Iowa state University
}

A method for determining the location and possible existence of symmetry elements in patterson superposition maps has been developed. A comparison of the original superposition map and a superposition map operated on by the symmetry element gives possible translations to the location of the symmetry element. A reciprocal space approach using structure factor-like quantities obtained from the Fourier transform of the superposition function is then used to determine the "best" location of the symmetry element. Constraints based upon the space group requirements are also used as a check on the locations. The locations of the symmetry elements are used to modify the Fourier transform coefficients of the superposition function to give an approximation of the structure factors, which are then refined using the EG relation. The analysis of several compounds using this method is presented. Reciprocal space techniques for locating multiple images in the superposition function are also presented, along with methods to remove 
the effect of multiple images in the Fourier transform coefficients of the superposition map.

In addition, crystallographic studies of the extended chain structure of $\left(\mathrm{NHC}_{5} \mathrm{H}_{5}\right) \mathrm{SbI}_{4}$ and of the twinning method of the orthorhombic form of the high-T $T_{C}$ superconductor $\mathrm{YBa}_{2} \mathrm{Cu}_{3} \mathrm{O}_{7-\mathrm{x}}$ are presented. 


\title{
A reciprocal space approach for locating symmetry elements in Patterson superposition maps
}

\author{
by \\ Thomas L. Hendrixson \\ A Dissertation submitted to the \\ Graduate Faculty in Partial Fulfillment of the \\ Requirements for the Degree of \\ DOCTOR OF PHILOSOPHY \\ Department: Chemistry \\ Major: Physical Chemistry
}

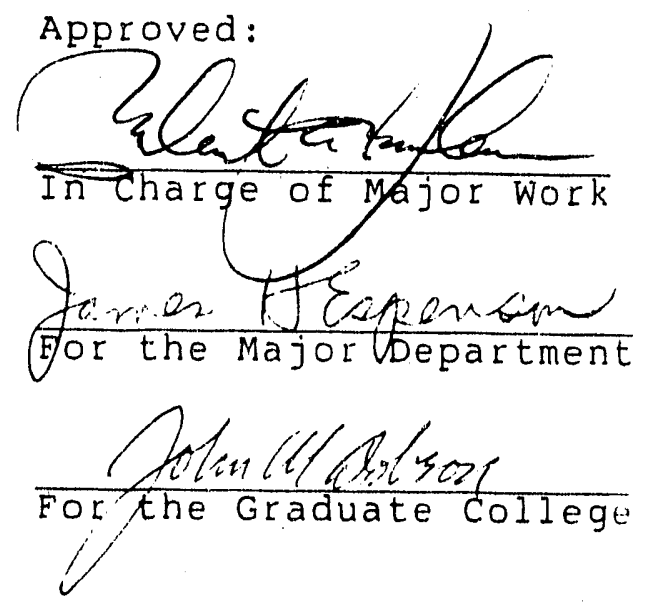

Iowa State University
Ames, Iowa 
TABLE OF CONTENTS

\section{PAGE}

CHAPTER 1. INTRODUCTION

CHAPTER 2. THE PHASE PROBLEÝ AND ITS SOLUTION 4

A Physical Description of the Problem 4

Direct Methods

Patterson Methods

The EG Relationship

CHAPTER 3. SYMMETRY ELEMENT LOCATION

The Superposition Map as a Pseudo-electron

Density Map

The Location of symmetry Elements in

Superposition Maps

Location of Multiple Images

CHAPTER 4. DETAILS OF THE HYPAD PROCEDURE

HYPAD

Graphics Programs

CHAPTER 5. APPLICATION OF HYPAD TO THE SOLUTION

OF KNOWN AND UNKNOWN STRUCTURES

HYPAD solution of $\mathrm{Cr}(\mathrm{CO})_{3} \cdot \mathrm{C}_{6} \mathrm{H}_{6}$

HYPAD solution of $\mathrm{FeP}_{2} \mathrm{OC}_{32} \mathrm{H}_{31} \mathrm{I}$

HYPAD Solution of $\mathrm{Ni}\left(\mathrm{C}_{6} \mathrm{O}_{3} \mathrm{H}_{9}\right)_{2} \mathrm{Cl}_{2}$

HYPAD solution of $\left(\mathrm{NHC}_{5} \mathrm{H}_{5}\right)_{2} \mathrm{I}_{10}$

HYPAD Solution of $\mathrm{Cu}\left(\mathrm{C}_{6} \mathrm{O}_{3} \mathrm{H}_{9}\right) \mathrm{Cl}_{2}$

CHAPTER 6. CONCLUSION 


\begin{tabular}{|c|c|c|}
\hline APPENDIX A. KEYWORD OPTIONS IN & N HYPAD & 110 \\
\hline GCALC.INP & & 111 \\
\hline LOCSYM. INP & & 112 \\
\hline MULTIM. INP & & 115 \\
\hline FOURIER. INP & & 115 \\
\hline PRESEARCH. INP & & 119 \\
\hline SEARCH. INP & & 120 \\
\hline QPLANES.INP & & 124 \\
\hline QROTLINE. INP & & 126 \\
\hline QSPLOT.INP & & 127 \\
\hline QDATA.INP & & 128 \\
\hline Sample Input Files & & 129 \\
\hline LOCSYM. INP & & 129 \\
\hline FOURIER. INP & & 130 \\
\hline ArPENDIX B. CRYSTAL STRUCTURE & DETERMINATIONS & 131 \\
\hline Structure Determination of ( & $\left(\mathrm{NHC}_{5} \mathrm{H}_{5}\right) \mathrm{SbI}_{4}$ & 131 \\
\hline Introduction & & 131 \\
\hline Experimental & & 132 \\
\hline Solution and Refinement & & 134 \\
\hline Discussion & & 135 \\
\hline Structure Determination of $P$ & $\mathrm{PO}_{4} \mathrm{C}_{2}{ }_{4} \mathrm{H}_{25}$ & 144 \\
\hline Experimental & & 144 \\
\hline Solution and Refinement & & 146 \\
\hline Structure Determination of $P$ & $\mathrm{PO}_{2} \mathrm{C}_{6} \mathrm{H}_{11}$ & 154 \\
\hline Experimental & & 154 \\
\hline Solution and Refinement & & 160 \\
\hline
\end{tabular}


Structure Determination of $\left(\mathrm{NHC}_{5} \mathrm{H}_{5}\right)_{2} \mathrm{I}_{10}$

$\begin{array}{ll}\text { Experimental } & 161\end{array}$

Solution and Refinement 162

Twinning Effects in $\mathrm{YBa}_{2} \mathrm{Cu}_{3} \mathrm{O}_{7-\mathrm{x}} \quad 166$

$\begin{array}{ll}\text { Crystallographic studies } & 166\end{array}$

Twin Modeling $\quad 167$

Discussion ? 172

$\begin{array}{ll}\text { LITERATURE CITED } & 183\end{array}$

$\begin{array}{ll}\text { ACKNOWLEDGEMENTS } & 186\end{array}$ 
CHAPTER 1. INTRODUCTION

In 1934, A. L. Patterson devised a function, now known as the Patterson function, which consists of all of tine interatomic vectors in the unit cell and can be obtained by Fourier transforming the observed intensities of the Bragg refluctions. Until the early 1950s, almost all crystal structures were solved via analysis of this function. The positions of atoms in a structure were usually obtained by examination of two-dimensional projections of the patterson function for characteristic interatomic vectors.

A new era dawned in 1952 with the development of a series of equations which showed that, theoretically, complete crystal structures could be obtained from the direct manipulation of the Bragg reflections. Such procedures became known as "direct methods". Much of the Initial popularity of direct methods procedures was due to their ability to solve the nearly-equal atom structures, which are difficult to solve using patterson methods. Today, the direct methods approach is quite often used in the first attempt at solving a crystal structure. While for many structures, direct methods will furnish at least a partial solution, there are still many structures that resist solution by the direct methods approach. It is these structures that have encouriged a renaissance in the development of Patterson method 
techniques by several research groups, notably including this one. Many of the groups have focused their efforts on the development of procedures that make use of known molecular fragments. However, this group has focused its efforts primarily on the development of ab initio methods, where no prior knowledge of the structure is required. Previously, indirect use of the space group symmetry was made by using Harker vectors and planes in these methods. A new procedure winch directly makes use of the space group symmetry has been developed and automated in a series of computer programs called HYPAD.

In any structure determination, the primary goal is the determination of the positional and thermal parameters of the atoms present in the structure. These parameters affect the amplitude and phase of the scattered wave. The amplitude of the scattered wave is proportional to the observed intensity of the scattered wave, but the phase is not measurable experimentally. The need to determine the phases of the scattered waves is often referred to as the "phase problem" in X-ray crystallography. A brief description of the phase problem is given in Chapter 2 , along with discussions of the approaches used in solving the phase problem; direct methods, Patterson, and Patterson superposition techniques.

Chapter 3 is devoted to the theory of the use of spare group symmetry in patterson superposition maps as utilized 
In HYPAD. Reciprocal space approaches for the location of symmetry lements and the location of, and correction for, multiple images in superposition maps are developed. A brief description of the programs avallable in HYPAD is given in chapter 4. The programs in HYPAD have been tested using several real sicuctures, some of which were known previously and some which were unknown at the time. These structures are discussed in Chapter 5. Chapter 6 contains a summary of the viability of the HYPAD approach, and a discussion of possible modifications to HYPAD is given in Chapter 7 .

Appendix A contains a listing of the keywords used in the various input files for HYPAD. A discussion of crystal structures completed using methods other than HYPAD are also listed in Appendix B. 
In every $x$-ray diffraction structure determination, the objective is the determination of the absolute phase shifts undergone by $x$-rays when they are scattered by a crystalline solid. This is what is known in crystallography as "the phase problem".

\section{A Physical Description of the Problem}

In a single crystal $x-r a y$ experiment, the incident $x-r a y$ beam interacts with the crystal resulting in a diffracted (or reflected) beam. Being an electromagnetic plane wave, the incident beam can be represented mathematically by $\psi(r)=\psi_{0} \exp \left(2 \pi i s_{0} \cdot r / \lambda\right)$,

where $\psi_{0}$ is the amplitude of the wave and $s_{0}$ is a unit vector in the direction of the propagation of the incident beam. For $x$-radiation, the wavelength, $\lambda$, is on the order of $1 \AA$, which is comparable to the spacing between atoms in a crystalline material. Therefore, a crystal will act as a diffraction grating when it is placed in a beam of $x$-rays. Because $\mathrm{x}$-rays are scattered most effectively by the electrons in the crystil, those crystals having larger concentrations of electrons will scatter the best. Each electron will scatter X-rays in every dizcotion, and thus, 
the $j^{\text {th }}$ electron will produce a distribution of scattered waves.

$$
\psi_{j}(r)=f_{j} \exp (2 \pi i s \cdot r / \lambda) .
$$

where $s$ is a unit vector in the direction of the scattered wave. In gerezal, the direction of s is different from that off $s_{0}$. The scattering amplitude for a single electron, $f_{j}$, falls of as the scattering angle increases.

In a diffraction experiment, the intensities of the scattered waves are measured. Each scattered ivave is the composite of the scattered waves from all of the individual ellectrons in the crystal, and is formed by the superposition of these waves. The individual waves can either reinforce each other ot interfere with each other. If two parallel in-phase incident waves, having a wavelength of $\lambda$, are scattered by parallel pianes in the crystal, separated by an interp: zne distance d, then they will scatter in-phase only for specific scattering angles $\theta$. Mathematically, this is giver by Bragg"s Law.

$$
\lambda=2 d \sin \theta \text {. }
$$

Similar phase relationships can be obtained when scattering from a distribution of electrons is considered. suppose there a-e two scattering centers, $c_{1}$ and $c_{2}$, as shown in Figure 2.1. These scattering centers can be either individual electrons or small lacalized distributions of electrons (e.g., atoms). For a center $C_{j}$, the path 


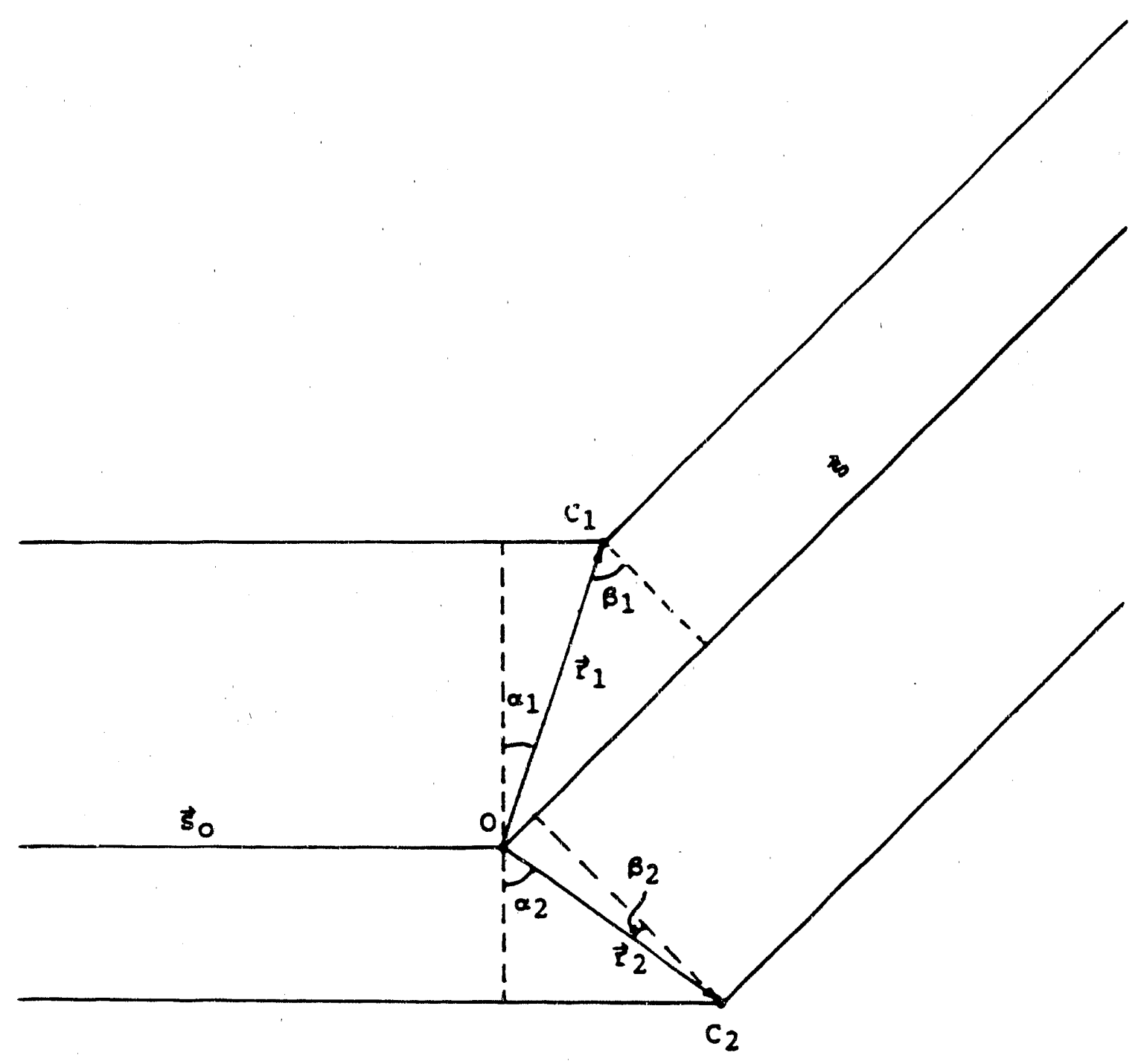

Figure 2.1. Schematic diagram for scattering from two poines $C_{1}$ and $C_{2}$ 
difference between a wave scattered by the center anc a wave scattered by an arbitrary origin 0 is given by

$$
\Delta_{j}=a_{j}-a_{j o}=\left|r_{j}\right|\left(\sin \beta_{j}-\sin \alpha_{j}\right) \text {. }
$$

This gives a phase difference of

$$
2 \pi \Delta_{j}=2 \pi\left(s-s_{o}\right) \cdot r_{j} / \lambda=2 \pi h_{1} \cdot r_{j}
$$

where $h$ is usually referred to as the diffraction vector.

The composite of the scattered waves is given by the structure factor, $F_{h}$, and is the sum of the individual scattering distributions

$$
\begin{aligned}
F_{h} & =\sum_{k}^{N_{e}} \psi_{k}(h)=\sum_{k}^{N_{e}} f_{j} \exp \left(2 \pi i h \cdot r_{k}\right) \\
& =\sum_{j}^{N_{a}} f_{j} \exp \left(2 \pi i h \cdot r_{j}\right),
\end{aligned}
$$

where $\mathrm{Ne}_{e}$ is the number of electrons in the unit cell, $\mathrm{Na}$ is the number of atoms in the unit cell and $f_{j}$ is the atomic scattering factor. The magnitude of $F_{h},\left|F_{h}\right|$, is related to the extent to which the atoms in the crystal scatter inphase for a particular reflection (i.e., the relative phase difierences of the diffracted waves), and is independent of the absolute phase of the reflection.

The electron density of a structure, $p(r)$, can be written as the Fourier transform of the structure factors

$$
\rho(\boldsymbol{r})=\int F_{h} \exp (-2 \pi i h \cdot r) d h,
$$

where the integral is over the entire space of wave vectors $h$, and $\left|F_{h}\right|^{2}$ is proportional to the observed intensity, $I_{h}$. since, in general, the structure factors are complex 
numbers, they can be expressed in terms of a phase and a magnitude

$$
\begin{aligned}
F_{\mathbf{h}} & =\left|F_{h}\right| \exp \left(2 \pi i \phi_{h}\right) \\
& =A_{h}+i B_{h} .
\end{aligned}
$$

Since, in practice, the integral in equation (2.7) is evaluated as a summation over a finite number of discrete points having integer values of $h=(h, k, l)$, the electron density can be rewritten as

$$
\rho(r)=\frac{1}{V} \sum_{h}\left|F_{h}\right| \exp \left(i \phi_{h}-2 \pi i h \cdot r\right) .
$$

Thus, if both the phase and the magnitude of all of the structure ractors are known, ihen complete information about the structure can be obtained. However, the phases of the structure factors, unlike the magnitudes, can not be obtained directly from a diffraction experiment. This loss of phase information is referred to as the "phase problem" in crystallography.

For a structure having a reasonable amount of complexity, there are essentially two basic methods to use to solve the phase problem; a real space method and a reciprocal space method. The remainder of this chapter is devoted to a brief discussion of the background and application of these methods. 
Direct Methods

The term "direct methods" is usually taken to mean those methods which attempt to derive the phases of the structure factors using only the intensity information. The birth of direct methods can be traced back to the iate 1940 s with the development of the Harker-kasper inequalities ${ }^{2}$ and the Karle-Hauptman determinantal inequalities ${ }^{3}$, which are based on the fact that the electron density is positive everywhere. While soma very simple centrosymmetric structures could be solved using these inequalities, they made very little impact in the practical solution of crystal structures.

In addition to the condition of non-negativity of the electron density, the concept of atomicity (i.e., the electron density can be resolved into separate regions representing atoms) can be imposed. This led to the derivation in 1952 of what is now known as the $\Sigma_{2}$ relation by sayre ${ }^{4}$, and independently by $\operatorname{Cochran}^{5}$ and zachariasen 6 . If the electron density function of a structure which consists of equal resolved atoms is squared, then the result looks similar to the original electron density function. The peaks in the squared function will be sharper and higher, but they will be in the same positions as in the original function. In terms of reciprocal space quantities, the $s_{2}$ relation is given by, 


$$
E_{h}=k\left\langle E_{h-k} E_{k}>_{k},\right.
$$

where \langle\rangle$_{k}$ represents the average over a complete set of vectors $k$, and the $E_{h}$ are the normalized structure factors. The vectors $h, k$, and $h-k$ form a closed triangle, or vector triplet, in reciprocal space.

Much of the early application of direct methods centered around the solution of centrosymmetric crystals. For centrosynmetric crystals, equation (2.10) becomes

$$
s(h) \simeq s\left[\sum_{k} s(k) s(h-k)\right],
$$

where $\approx$ means "probably equals", $\mathbf{s}(\mathbf{h})$ is the sign of $F_{h}$, and the terms on the right-hand side of equation (2.11) are pairs of known signs which give an indication for $s(h)$. The probability that $s(h)$ is positive, given many contributor pairs was first derived by Cochran and woolfson 7 and is given by

$$
P_{+}(h)=\frac{1}{2}+\frac{1}{2} \tanh \left[\frac{\sigma_{3}}{\frac{3}{2}}\left|E_{h}\right| \sum_{k} E_{k} E_{h-k}\right] ，
$$

where on is

$$
\sigma_{n}=\sum_{j}\left(z_{j}\right)^{n}
$$

and $z_{j}$ is the atomic number of the $j^{\text {th }}$ atom.

It was not until a few years later that a probability distribution for the general phase relationship

$$
\phi_{\mathbf{h}}=\phi_{\mathbf{k}}+\phi_{\mathbf{h}-\mathbf{k}}
$$

was developed by Cochran 8 


$$
P\left(\phi_{h}\right)=\frac{1}{2 \pi I_{0}\left(k_{h, k}\right)} \exp \left(k_{h, k} \cos \left(\phi_{h}-\phi_{k}-\phi_{h-k}\right)\right),
$$

where

$$
K_{h, k}=\frac{\sigma_{3}}{\sigma_{2}^{3 / 2}}\left|E_{h} E_{k} E_{h-k}\right|
$$

and $I_{0}\left(K_{h}, k\right)$ is a modifled Bessel function,

$$
I_{0}(x)=\sum_{n=1}^{\infty} \frac{x^{2 n}}{2^{2 n}((2 n) !)^{2}}
$$

The probability expressions quantify what would otherwise be a qualitative manipulation of the phases.

In 1956, Karle and Hauptman ${ }^{9}$ introduced an equation which provides an estimate for $\phi(h)$ when several pairs of known phases are available. This was given in the form of the tangent formula which is

$$
\tan \phi_{h} \simeq \frac{\sum_{k} k_{h, k} \sin \left(\phi_{k}+\phi_{h-k}\right)}{\sum_{k} k_{h, k} \cos \left(\phi_{k}+\phi_{h-k}\right)} .
$$

These developments gave crystallographers the tools to solve some of the structures that had previously been difficult to solve, such as structures containing atoms of equal or nearly equal atomic number, and led to the development of several structural solution strategies. One of the earliest such strategies was symbolic Addition, developed by Karle and Karle10. In symbolic Addition, algebraic symbols are assigned to the phases of a group of reflections, known as the starting set. The starting set is 
chosen by taking those structure factors that have large $\left|E_{h}\right|$ and that can be combined with other members of the starting set to give phase estimates of many other reflections. Once a consistent set of symbolic phases has been obtained, the values of the phases can be calculated. Probably the most widely used direct methods approach is MULTAN, developed by Gernain, Main and Woolfsont1. This approach is similar to that of symbolic Addition, except that. numerical values for the phases are substituted for symbols at the outset. These numeric phases are then refined using equation (2.10) and new estimates of the phases are calculated using equation (2.18).

since the introduction of MULTAN, there have been many advances in the direct methods strategy. With the addition of various concepts, such as magic integers 12 , the neighborhood principle13, quartets $13-17$, random starting phases 18 , and the maximum-entropy method19-21 to name a few, many fairly complex compounds can be solved using direct methods. However, there still remain numerous compounds that resist solution via direct methods. This has prompted a renewal of interest in real space, or patterson methods.

Patterson Methods

Although the connection between Fourier theory and $\mathrm{X}-\mathrm{ray}$ diffraction had been well known since 1913, when it was 
first noted at a solvay conference, it was was not widely used until 1934, when A. L. Patterson introduced a Fourier series explession, now known as the patterson function 22 , which could be calculated directly from the observed intensities

$$
\begin{aligned}
P(u) & =\int \rho(r) \rho(r+u) d r \\
& =\sum_{h}\left|F_{h}\right|^{2} \exp (2 \pi i h \cdot u) .
\end{aligned}
$$

The sqllare of the magnitude of the structure factors can be written as

$$
\begin{aligned}
\left|F_{h}\right|^{2} & =F_{h} F_{-h} \\
& =\sum_{j}^{\|} \sum_{k}^{N} f_{j} f_{k} \exp \left(2 \pi i h \cdot\left(r_{j}-r_{k}\right)\right) .
\end{aligned}
$$

Thus, peaks in the Patterson function occur when two atoms are separated by the vector $u$. Furthermore, the magnitude of a peak is proportional to a contribution of $z_{j} z_{k}$ for atoms $f$ and $k$ such that $r_{j}-r_{k}=u$. The concept that the patterson function repr sents all of the interatomic vectors in the unit cell is importart to the understanding and interpretation of the function.

In a vector set notation where $\left\{a_{i}-a_{1}\right\}, i=1, N$, represents the set of interatomic vectors between atom 1 and all the atoms in the unit cell (i.e., one image of the structure), the patterson function is given as

$$
\begin{aligned}
{[P(u)] } & \equiv\left\{a_{i}-a_{1}\right\} \cup\left\{a_{i}-a_{2}\right\} \cup \ldots \cup\left\{a_{1}-a_{N}\right\} \\
& \equiv\left\{a_{i}-a_{j}\right\}, \quad 1, j=1, N .
\end{aligned}
$$


Thus, for a structure containing $N$ atoms, the patterson function contains $\mathrm{N}$ images of the structure, superimposed upon one another. This can make interpretation of the Patterson function difficult, even for simple structures.

To 1llustrate, consider the hypothetical structure shown in Figure 2.2, ("Elephantine"23). This structure contains 4 heavier atoms, such as iron, per molecule and a long chain of carbons. In the patterson map generated by this molecule, the dominant images are the iron images (i.e., those images formed by vectors having iron atoms at the tail of the vector). The patterson map shown in Figure 2.3 is composed of only the iron lmages and thus contains 16 images of the structure. One can see that even for this relatively simple structure, the patterson map is very complex.

In 1936, the concept of symmetry was introduced into Patterson analysis by $D$. Harker by the use of Harker vectors 24 . Harker vectors are interatomic vectors between symmetry-related atoms. The Harker vectors for a given space group have the same general form, regardless of the actual position of the atoms in question. Table 2.1 lists the Harker vectors for the space group $\mathrm{P} 21 / \mathrm{C}$. For structures containing only a few heavier atoms, which produce the more prominent peaks in the Patterson map, the Harker vectors and Harker planes can be used to infer positional information of some of the atoms. 


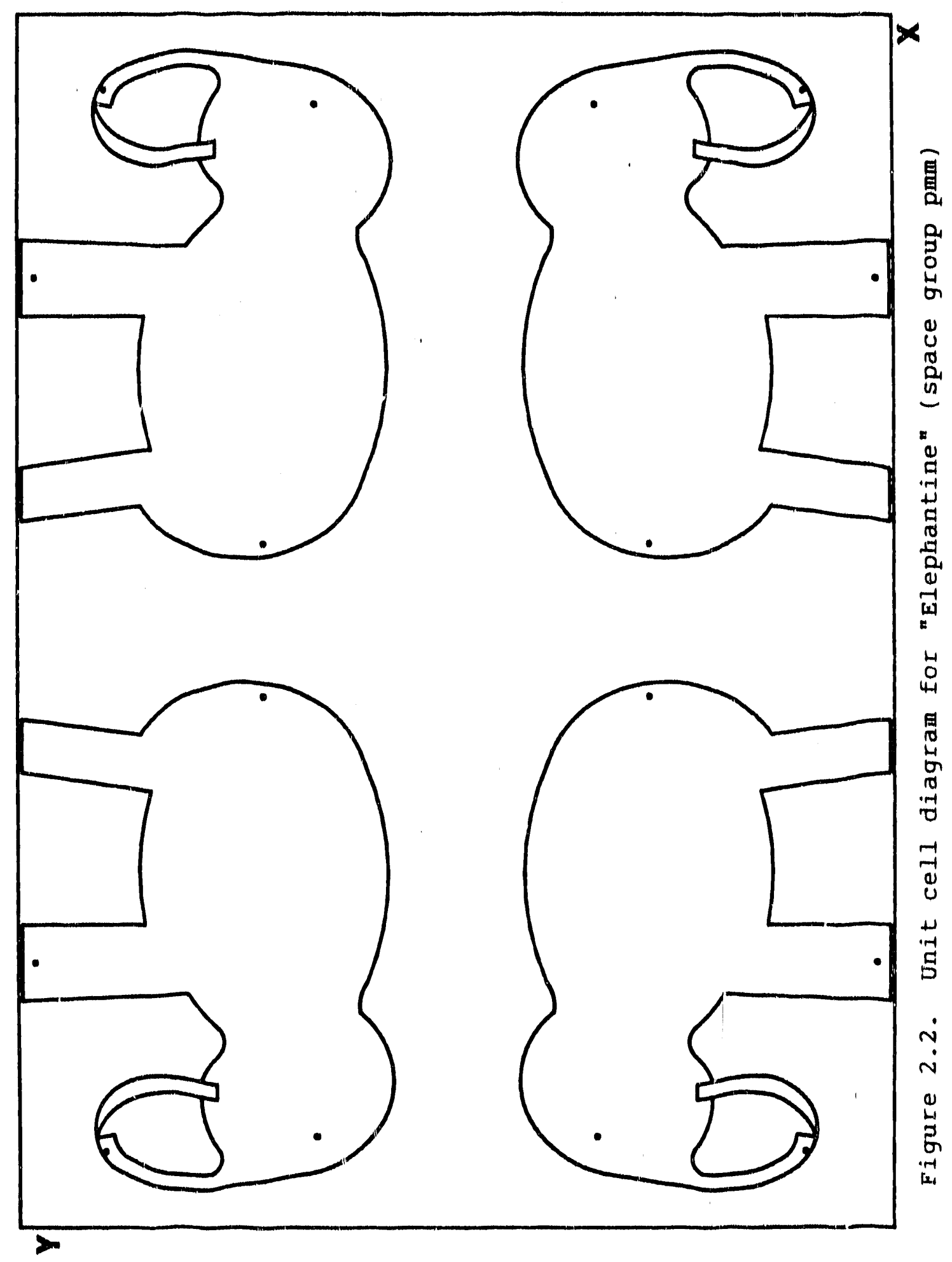



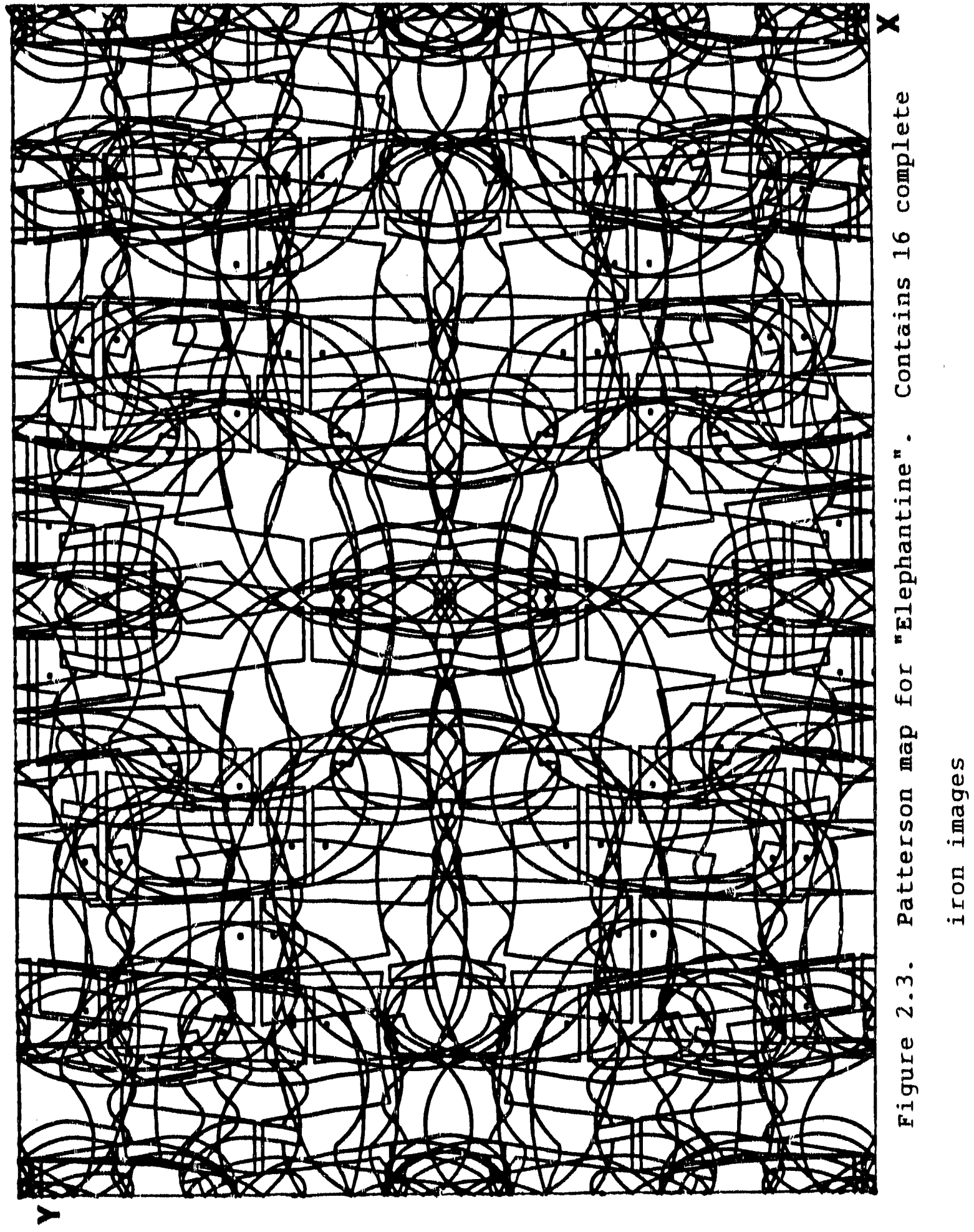


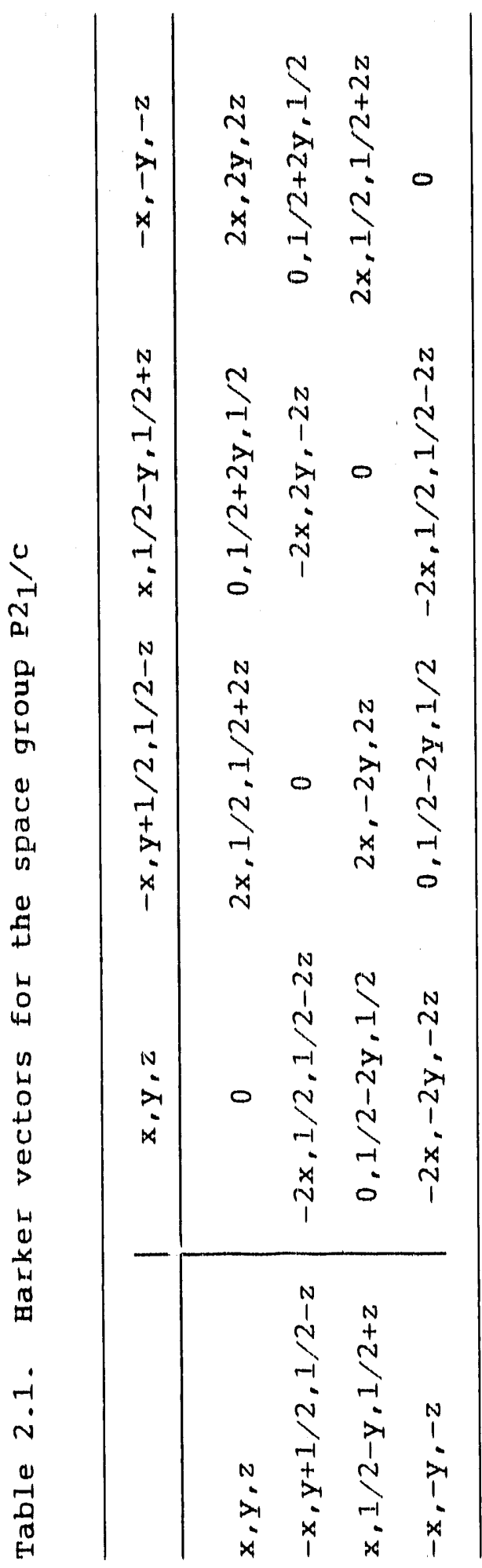


However, an ever-present problem in Harker analysis is that the 21 -screw vector, for example, of one atom may accidentally combine with the glide vector of an unrelated atom to give the position of a peak which is actually present in the Patterson map but is not the inversion vector for elther atom. A method, referred to as vector Verification 25 , has been developed to help combat this problem. In vector verification, the interatomic vectors based on the atomic positions obtained from the Harker analysis are calculated and then verified by checking to see if they are in the patterson map.

Quite often, if the structure contains a small number of heavy atoms, the positions of these atoms can be used to estimate the phases of the $F_{h}$. Calculation of an electron density map using these phases will then provide possible positions for the rest of the structure. Of course, the assumption inherent in this approach is that the phases from the partial structure are close to the phases obtained from the entire structure. Obviously, this is not always the case.

In 1950, it was discovered by Clastre and Gay 26 and Garrido27 that the superposition of a shifted Patterson map onto an unshifted map resulted in the superposition of a relatively small number of vectors. This set of vectors is referred to as the "reduced vector set". This superposition of Patterson maps results in the partial deconvolution of 
the Pattersom function. Much of the early systematic arallysils off the superposition was done by Buerger 28 " who showed that applitication of this procedure could lead to the decomallution of the Pattersom map into one image.

This cam be best illustrated in terms of the vector set motation introduced earlier. The superposition can be thougint of as the intersection off the set of unshifted vectors and the set of shifted vectors. If the shift vector s is a simglle "i.e.e unique》 interatomic vector, such that $=\left(\mathbf{a}_{2}-\mathbf{a}_{1} \|\right)$. them the superposition can be witen as

$$
\begin{aligned}
& \|\mathbb{U}(\| \mathbf{r})\| \equiv \|\left\{a_{i}-a_{j}\|+\| a_{2}-a_{1}\|\| m\|\| a_{i}-a_{j}\|\|\right. \\
& =\|\| a_{i}-a_{1}\left\|+\left\{a_{2}-a_{j}\right\}\right\| m \|\left\{a_{i}-a_{j} \|\right\} \\
& \equiv\left\{a_{i}-a_{1}\|U\| a_{2}-a_{j}\|,\| i, j=1, \mathbb{N}\right. \text {. }
\end{aligned}
$$

Thus, tho images remain after the superposition. One is an image: as "viewedin from the attom at $\mathbf{a}_{1}$ and the other is the inverse off the image as "vilewed" from the atom at a . These 任 wo inages; are rellated by an inversion center at $\| a_{2}$ ayll/2. Figure 2.4 shows the result of a superposition using a single vector for "Ellepharitime". If a second vector, $\mathbf{s}_{1}=$ $a_{3}$ - all is chosem to use in am additional superposition, then the result is

$$
\begin{aligned}
& \| M\left((\mathbf{c}) \rrbracket \equiv\left\|\forall \mathbf{a}_{i}-\mathbf{a}_{j}\right\|+\left(\mathbf{a}_{2}-\mathbf{a}_{1}\right)\|m\|\left\{\mathbf{a}_{i}-\mathbf{a}_{j} \|+\left(\mathbf{a}_{3}\right.\right.\right. \\
& -\mathbf{a}_{1}\left\|\rrbracket m_{i}\right\|\left\{\mathbf{a}_{i}-\mathbf{a}_{j} \Downarrow \|\right. \\
& \equiv \|\left\{a_{i}-a_{\mathbb{1}}\|\|, \quad i, j=\mathbb{1}, \mathbb{N}\right. \text {. }
\end{aligned}
$$

Thus, theoretically, one image of the structure can be obtained after two superpositions using single vectors. 


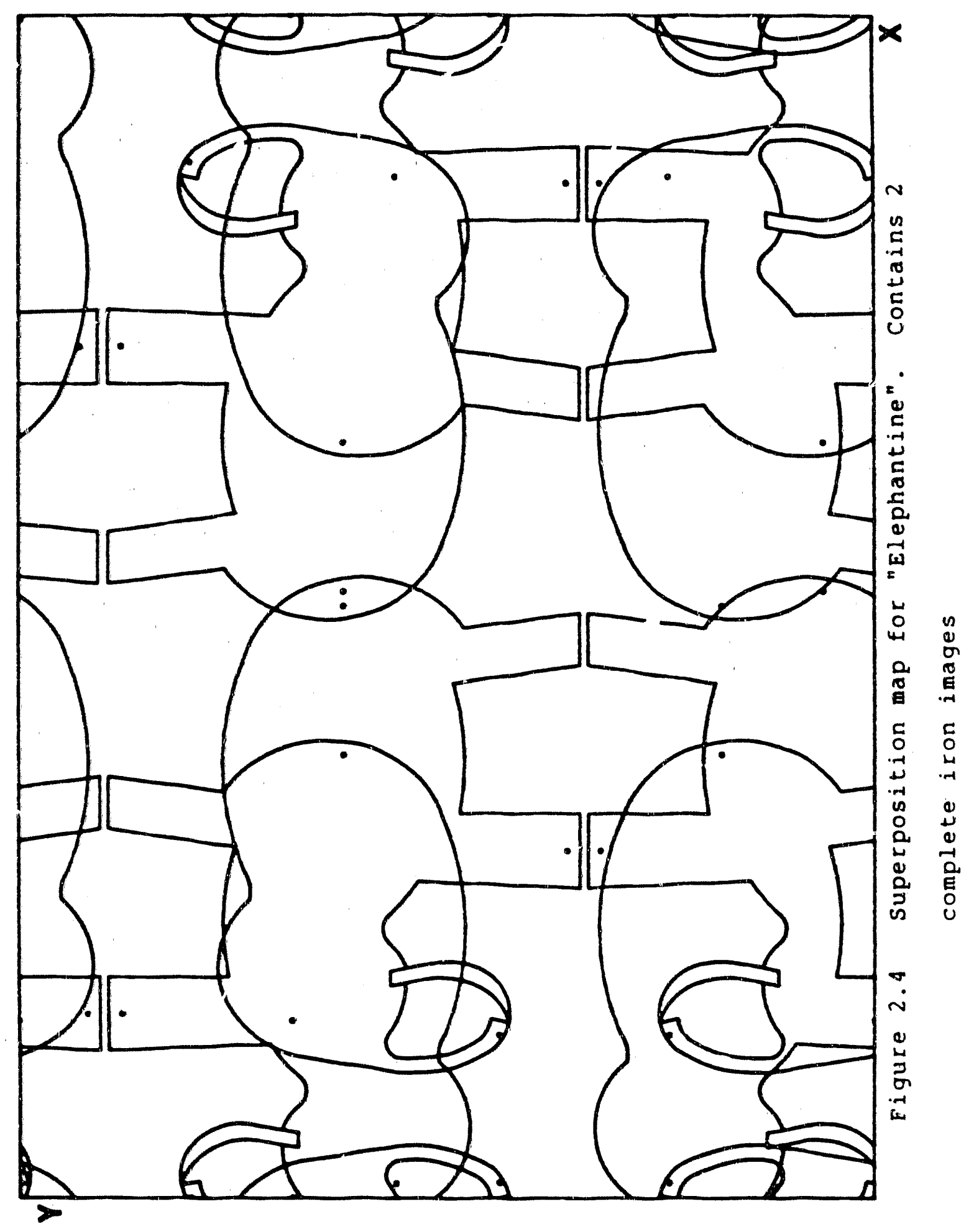


Unfortunately, since multiple vectors are easier to find in a Patterson map, they are more likely to be used in the superposition. This car cause difficulties because the superposition map will then contain multiple images. If the shift vector is multiple vector, $\mathbf{s}=\left(\mathbf{a}_{2}-\mathbf{a}_{1}\right)=\left(\mathbf{a}_{4}-\right.$ $\left.a_{3}\right)$, then the superposition is

$$
\begin{aligned}
{[M(r)]=\left\{a_{i}-a_{1}\right\} \cup\left\{a_{2}-a_{j}\right\} \cup\left\{a_{i}-a_{3}\right\} \cup\left\{a_{4}\right.} \\
\left.-a_{j}\right\}, i, j=1, N
\end{aligned}
$$

Two of the images are correctly oriented (from $a_{1}$ and $a_{3}$ ) and two of the images are inverse images (from $a_{2}$ and $a_{4}$ ).

There are a number of ways to do a Patterson superposition. The preferred way is do a "minimum" convolution of the patterson function and the shifted Patterson function,

$$
M(r)=\min (P(r), P(r+E)) \text {. }
$$

In practice, this is calculated by taking the point-wise minimum of $P(\mathbf{r})$ and $P(\mathbf{r}+\mathbf{s})$ at all points $r$ in the unit cell.

If the shift vector chosen for the superposition is an interatomic vector connecting atoms of different atomic number, then the vectors in the reduced vector set will have incorrect peak heights. If the shifted patterson is weighted by the ratio of the atomic numbers of the atoms at the head and tail of the shift vector, then the reduced vector set will have correct weights 29 . For example, if the 
shift vector is given by $s-\left(a_{2}-a_{1}\right)$ and $\tau-z_{1} / z_{2}$, then the weighted superposition function is given by

$M(r)=\min (P(r), \tau P(r+s))$.

If we refer to equation (2.22), a weighted superposition will produce heights for the vectors in the reduced vector set corresponding to the smaller of $z_{1} z_{1}$ and $\tau z_{1} z_{2}$, and $z_{2} z_{j}$ and $\tau z_{1} z_{j}$, respectively. As an example, in order for the vector ( $\left.a_{4}-a_{1}\right)$ to be appear in the superposition map, the vector $\left(a_{4}-a_{2}\right)$ must be shifted by $\left(a_{2}-a_{1}\right)$ in order to superimpose upon the vector $\left(a_{4}-a_{1}\right)$ in the original map. In an unweighted superposition, the height of this vector would be the minimum of $z_{4} z_{2}$ and $z_{4} z_{1}$, or $z_{4} z_{2}$, which is the incorrect height for this vector. In a weighted superposition, the height would be the minimum of $\left(z_{1} / z_{2}\right) \star\left(z_{4} z_{2}\right)-z_{1} z_{4}$ and $z_{1} z_{4}$, or $z_{1} z_{4}$. Welghting the superposition has the effect of suppressing one image relative to the other image. Thus, weighted superpositions are done whenever the atomic numbers of the atoms of the shift vector can be estimated and the weighting factor would be different from unity.

The study of macromolecules such as proteins and viruses in the $1950 \mathrm{~s}$ led to the discovery that identical, but not crystallographically symmetry-related, units can often be found throughout the structure. This discovery prompted the development of a technique now known as Molecular Replacement 30 , where the locations and orientations of these 
units in electron density space could be calculated by locating the positions and orientations of the characteristic patterson space patterns. This method gave rise to the development of translational and rotational functions 31 which measure the overlap of the Patterson function and a copy of the Patterson which has been transformed about a non-crystallograplitic symmetry element. A method which is applicable when partial molecular geometry information is available was developed by Nordman ${ }^{32}$. In this approach, all of the interatomic vectors in the molecular fragment are calculated. This pattern of vectors is then rotated and translated in order to provide the best match with a portion of the patterson map. In the past decade, there has been a resurgence of interest in patterson and Patterson superposition methods. Many computer programs, such as ALCAMPS $33, \operatorname{SHELXS}^{33}, \operatorname{XFPS}^{34}$, IMPAS 35 , and HASSP 36 have appeared. However, for most crystallographers, Patterson techniques still remain the last alternative, something to be tried only when direct methods techniques have falled completely, because the manipulations tend to be somewhat cumbersome.

The EG Relationship

One of the advantages of Patterson techniques is that they are easy to start. For a superposition, one simply 
needs to choose a vector in the map. However successive application of superpositions entails a large amount of risk since all vectors used in the superposition must come from the same image. On the other hand, with a good starting set of phases, direct methods techniques will often result in a solution. However, with a bad set of startirg phases, direct methods fail to provide a solution. Quite often, it can be difficult to find a good starting set of phases. It would be advantageous if a method could be found whereby the easy start of the Patterson methods and the stability of the direct methods - given a good start - are combined. Such an approach has been examined by this group previously37. Using the assumption that the product of the Patterson superposition function and the electron density function looks like the square of the electron density function, then the following relation, called the EG relationship, is obtained

$$
E_{h}=k<E_{k} G_{h-k}>_{k},
$$

where the $G_{h-k}$ aie the Fourier transform coefficients of the Patterson superposition map. Unlike the $E_{h}$, both the phase and the magnitude of the $G_{h}$ are known, thus enablirig a large number of terms to be included in equation (2.27) at the outset of a phase refinement. 
In the past, procedures that have attempted to find symmetry elements in superposition maps have been real space methods. Typically, the major focus of attention has been an examination of the characteristic Harker vectors in an attempt to locate the symmetry elements. What will be presented tiere is a reciprocal space approach.

The Superposition Map as a Pseudo-electron Density Map

There exist relationships between the normalized structure factors, $E_{h}$, which arise from the symmetry elements present in the unit cell. However, the Fourier transform coefficients of the superposition map, $G_{h}$, only have P1 symmetry, in general. Since the structure under study usually has higher symmetry than P1, it is desirable to transform the $G_{h}$ into some $G_{h}^{\prime}$, where the $G_{h}^{\prime}$ have symmetry relationships that arise from the symmetry elements present.

If we examine the ideal case where the structure consists of point atoms and a unique (i.e., single) vector is chosen as the superposition vector, the resulting superposition map would contain two images of the structure and no extraneous peaks. If we further add the condition that the superposition be weighted, then the superposition 
map will contain predominantiy one lmage of the structure, with the inverse image substantially reduced by comparison. since the origin of the superposition map lies on an atom, and, in general, the origin of the electron density map is defined relative to the symmetry elements, the superposition map can be considered not only to be a first approximation to an electron density map, but one with the origin in the wrong position. The coefficients $G_{h}$ would then be related to the $F_{h}$ by

$$
G_{h}=k F_{h} \exp \left(-2 \pi i h \cdot t_{o r g}\right),
$$

where torg is the vector from the location (in the superposition map) of the electron density map origin to the origin of the superposition map, and $k$ is a scale factor. Equation (3.1) can be rewritten as

$$
\begin{aligned}
F_{h} & =k G_{h} \exp \left(2 \pi i h \cdot t_{o r g}\right) \\
& =k G_{h}^{t_{\text {org }}},
\end{aligned}
$$

which produces the structure factor-like quantities, Ghorg. These new coefficients should have the desired symmetry relationships.

Therefore, once the location of the electron density map origin in the superposition map has been determined, the $G_{h}$ can be transformed using equation (3.2). The phases of the transformed coefficients can be used as a first approximation to the phases of the $F_{h}$ and these phases can 
In turn be further refined by use of the $\Sigma_{2}$ relation or the EG relation.

The Location of symmetry Elements in superposition Maps

Previous attempts at locating the position of symmetry elements have focused their efforts on examining the characteristic Harker vectors for solutions which occur frequently. One of the difficulties with this type of analysis is that the peak positions from the superposition map are approximate. This means that only rarely wlll exact matches be found; the user must declde on a tolerance within which matches will be considered "exact". However, if one could use the Fourler transform coefficients of the superposition map to determine the location of the symmetry element, then the problem of "how close is close" can be eliminated.

The unit cell of an alternate form of the elephant compound is represented in Figure 3.1. This form of the compound contains a glide line perpendicular to the $y$ axis at $y=1 / 2$. (The glide line reflects the image in the $y$ direction and translates $1 / 2$ of the unit cell in the $x$ direction.) Placement of the glide line at some arbitrary position $t$, as shown in Figure 3.2 , will generate the clasned image. If the glide line is incorrectly placed, then the dashed image will only partially overlap the the original, 


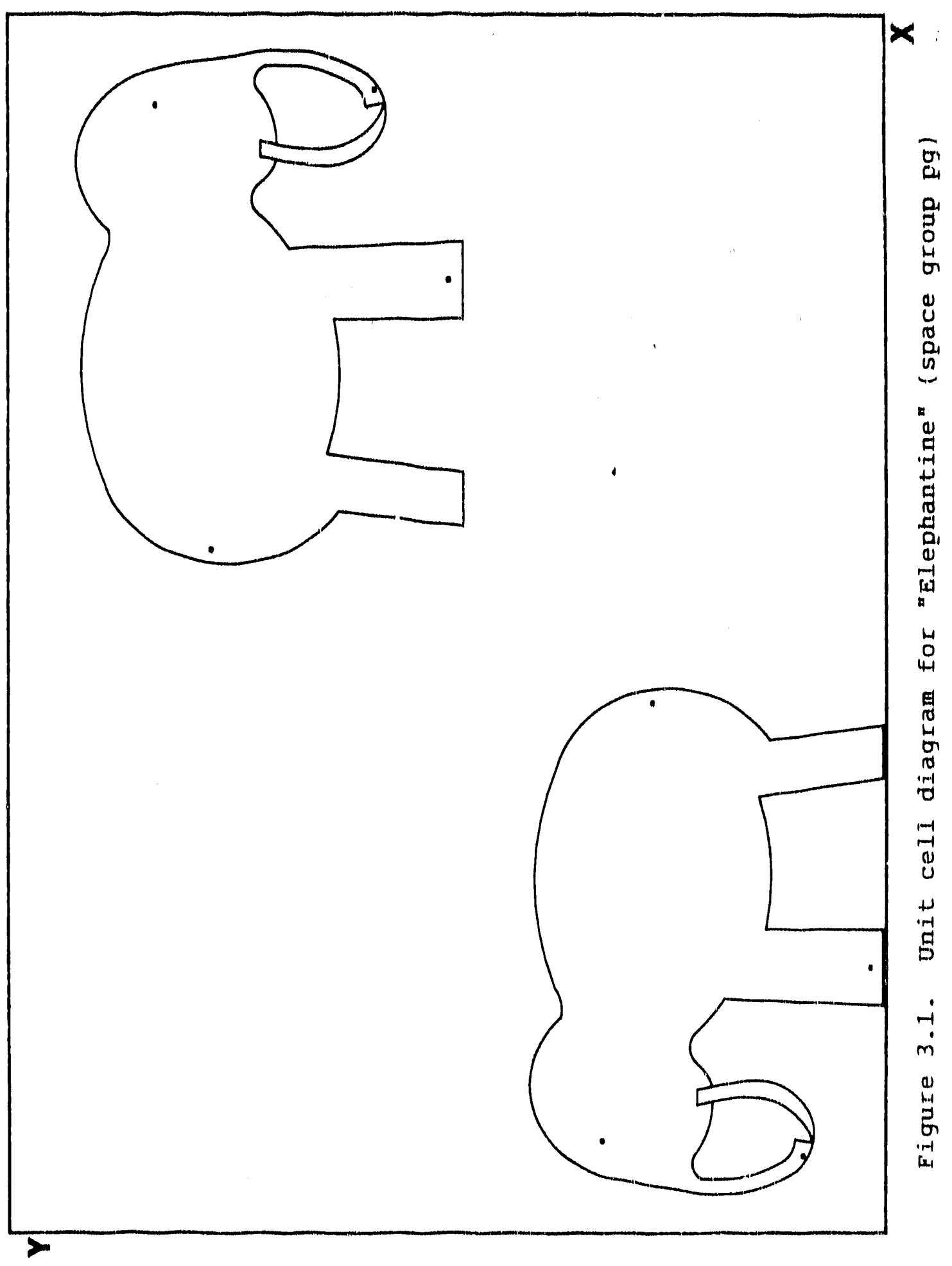




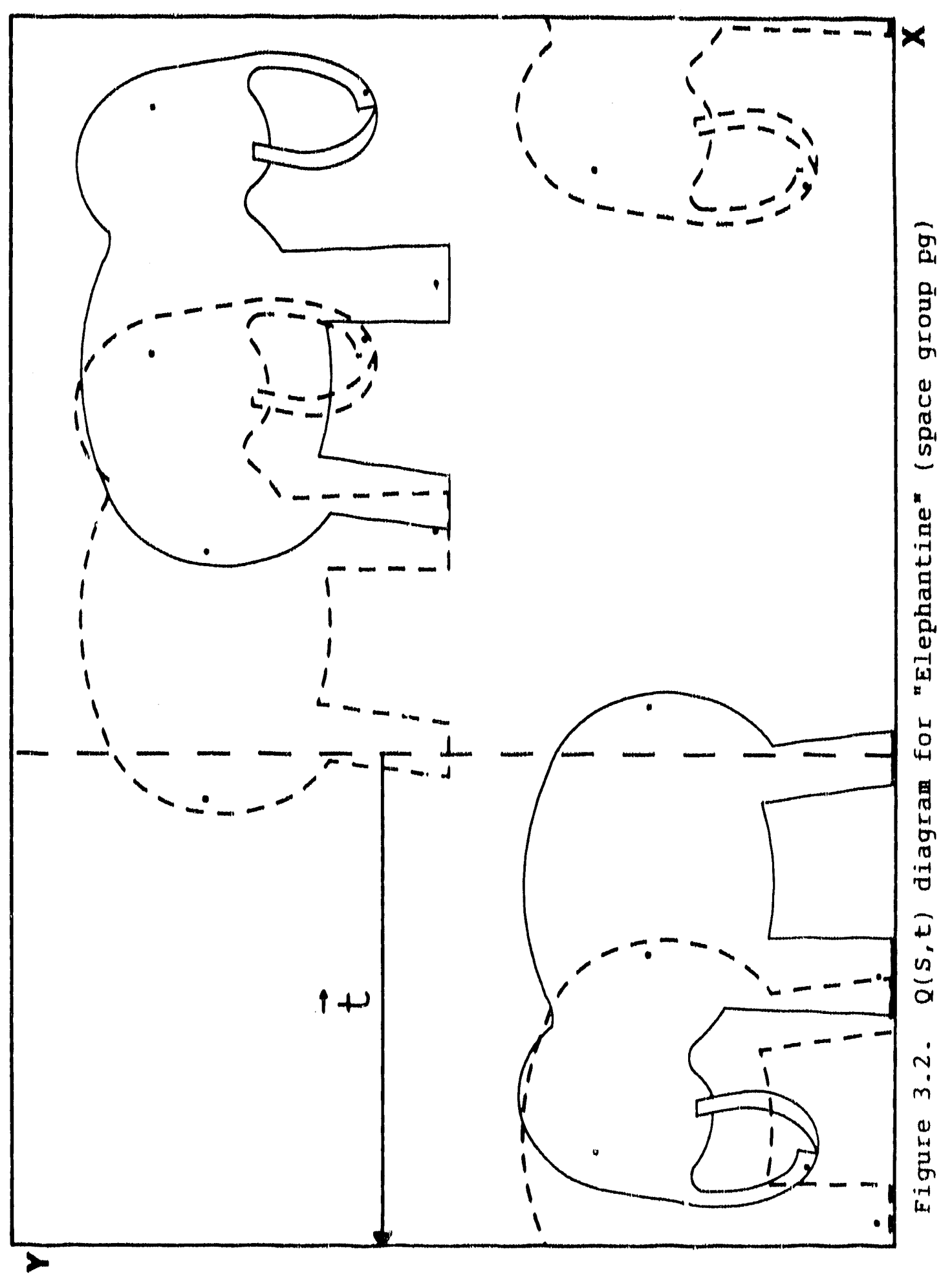


soldd lmage, le at all. However, when the glide line is placed in the correct location, the two lmages wlll overlap completely, maximizing the amount of overlap.

This overlap can bo expressed in terms of the product of the orlginal map and the map produced by the symmetry operator, and is given mathematicalily by

$$
Q(\hat{S}, t)=\int M(r \mid \hat{E}, 0) M(r \mid \hat{S}, t) d r \text {, }
$$

where $M(r \mid \hat{s}, t)$ is the map produced by placing the symmetry operator $\hat{S}$ at the position $t, \hat{E}$ is the ldentity operator, and the integral is over all space.

In general, a symmetry operator $\hat{s}$, located at a postion $t$ (with $t$ defined as shown in figure 3.2), can be written in terms of its rotational component, $R$, and its translational component, T. The result of a symmetry operator $\hat{s}$ operating on a point $r$ is given by

$$
\hat{S} r=R \cdot r+(R-I) \cdot t+T,
$$

where I is the ldentity matix. Using this definition, the rourler transform coefficients of $M(r \mid \hat{s}, t), H_{h}$, are given by

$$
\begin{aligned}
H_{h} & =\sum E_{j} \exp \left(2 \pi i h \cdot \hat{S} r_{j}\right) \\
& =\sum_{j} E_{j} \exp \left(2 \pi i h \cdot\left(R \cdot r_{j}+(R-I) \cdot t+I J\right)\right. \\
& =G_{h R} \exp (2 \pi i h \cdot((R-I) \cdot t+T)),
\end{aligned}
$$

where GhR is a Fourler transform coefficient of the original superposition map. Thus, the map produced by the symmetry operator can be expressed in terms of the ordginal map. 
Using the pourler transform of the $\operatorname{maps} M(\mathbf{r} \mid \hat{\mathrm{E}}, 0)$ and $M(r \mid \hat{s}, t)$, the expression for the overlap, $Q(\hat{s}, t)$ is

$$
\begin{aligned}
Q(\hat{S}, t) & =\sum_{h k} G_{h} H_{k} \int \exp (-2 \pi i(h+k) \cdot r) d r \\
& =\sum_{h} G_{h} H_{-h} .
\end{aligned}
$$

substituting the definition of the coefficients $\mathrm{H}_{\mathrm{h}}$ into equation $(3.6)$, the liesult

$$
\begin{aligned}
Q(\hat{S}, t)= & \sum_{h}\left|G_{h}\right|\left|G_{h}\right| \exp \left(1 \left(\alpha_{h}-\alpha_{h}+2 \pi h \cdot(1-R) \cdot t\right.\right. \\
& -(T)))
\end{aligned}
$$

is obtalned, where $h^{\prime}=h^{\prime} \cdot \mathbf{R}$.

Ideally, if the $G_{h}$ s had exact symmetry, then the term in the exponential would be zero for the correct translation and $Q(\hat{S}, t)$ would be a maximum,

$$
Q^{\max }(\hat{s}, t)=\sum_{h}\left|G_{h}\right|\left|G_{h}\right| \text {. }
$$

However, since the $G_{h}{ }^{\prime} s$ only have approximate symmetry, $Q(\hat{S}, t)$ will be less than $\left.\operatorname{Qmax}^{\mathrm{max}}, t\right)$, but wil1 sti11 11kely be a maximum for the correct translation. For the purposes of comparison, all $Q(\hat{S}, t)$ values will be referenced to $Q^{\max }(\hat{S}, t)$

Since $Q(\hat{s}, t)$ is a maximum when the term in the exponeutial is small, one can altornatively try to minimize the phase difference between $G_{h}$ and $G_{h}$. If we define a function 


$$
\begin{aligned}
S(\hat{S}, t)= & \frac{1}{N} \Sigma\left|G_{h}\right|\left|G_{h} \cdot\right|\left(\left(\alpha_{h}-\alpha_{h} \cdot\right) / 2 \pi+h \cdot(I-R) \cdot t\right. \\
& -h \cdot T)^{2},
\end{aligned}
$$

where $\left|G_{h}\right|\left|G_{h}\right|$ is included as a weighting function and $N$ is the number of terms in the summation, it should be a minimum for the correct translation.

In practice, both the $Q$ and the $s$ functions are evaluated. $Q$ is computationally faster to evaluate, but the $S$ function is more sensitive to the presence or absence of a symmetry operation. To determine the "best" location from a group of cholces, a figure-of-merit (FOM) is used. The figure-of-merit is given by

$$
\operatorname{FOM}(\hat{s}, t)=\frac{1}{\varepsilon}\left[\frac{Q(\hat{s}, t)}{Q_{\text {group }}^{\max }}+\frac{S_{g \text { roup }}^{\min }}{S(\hat{s}, t)}\right]
$$

where Qmax smaxidp is the minimum $s(\hat{s}, t)$ value in the group, and $\varepsilon$ is a parameter which takes into account the inability of the superposition to completely remove the Patterson symmetry. The form of $\varepsilon$ wis established by trial-and-error: for $t=$ $\left((0,0,0),\left(0,0, \frac{1}{2}\right),\left(0, \frac{1}{2}, 0\right),\left(0, \frac{1}{2}, \frac{1}{2}\right),\left(\frac{1}{2}, 0,0\right),\left(\frac{1}{2}, 0, \frac{1}{2}\right)\right.$, $\left.\left(\frac{1}{2}, \frac{1}{2}, 0\right),\left(\frac{1}{2}, \frac{1}{2}, \frac{1}{2}\right)\right)$,

$$
\varepsilon=1+\frac{\min (5-1,4)}{4},
$$

where $f$ is the number of superposition vectors used to create the map, otherwise $\varepsilon=1$. 
In the preceding derivations, it has been assumed that the symmetry elements of a space exist independently of one amother. However, for a given space group, the presence of a symmetry ellement $\hat{s}_{1}$. located at a position $t_{1}$. may restuict the positions ( $t_{j}$ ), of other symmetry ellements, $\hat{s}_{j}$.

One of the pirperties of a giroup is that the product of two ellements of the giroup must be itself an ellement of the group: which means:

$$
\hat{s}_{1} s_{2}=\tilde{s}_{2} \mathbf{r} \text {. }
$$

Using the definition of a symmety operator given in equation ( 3.4$)$. the foll 10 ming tellion between the locations of the symmetry operators and the transilational components off the symmetry operators is abtained:

$$
\begin{gathered}
\mathbf{R}_{2} \cdot\left(\mathbf{R}_{1}-\mathbf{I}\right) \cdot \mathbf{t}_{1}+\left(\mathbf{R}_{2}-\mathbf{I}\right) \cdot \mathbf{t}_{2}-\left(\mathbf{R}_{3}-\mathbf{I}\right) \cdot \mathbf{t}_{3}=\mathbf{T}_{3} \\
-\mathbf{R}_{2} \mathbf{T}_{1}-\mathbf{T}_{2} .
\end{gathered}
$$

For the product of 3 symmetry operators, the amalogous expiresion

$$
\begin{array}{r}
\mathbf{R}_{3} \cdot \mathbf{R}_{2} \cdot\left(\mathbf{R}_{1}-\mathbf{I}\right) \cdot t_{1}+\mathbf{R}_{3} \cdot\left(\mathbf{R}_{2}-\mathbf{I}\right) \cdot t_{2}+\left(\mathbf{R}_{3}-\mathbf{I} \cdot \mathbf{t}_{3}-\left(\mathbf{R}_{4}\right.\right. \\
-\mathbf{I}) \cdot t_{4}=\mathbf{T}_{4}-\mathbf{R}_{3} \cdot \mathbf{R}_{2} \cdot \mathbf{T}_{1}-\mathbf{R}_{3} \cdot \mathbf{T}_{2}-\mathbf{T}_{3} .(3.14)
\end{array}
$$

is olbained.

Thus, evaluating the functions $Q(\hat{S}, t)$ and $s(\hat{S}, t)$ while constraining the locations of different symmetry operators to satisfy equation (3. I3) will enable the locations of the symmetry operators to be correctly determined. 
Location of Multiple Images

The Patterson superposition map is a partial deconvolution of the Patterson map. Only rarely does it approach a full deconvolution, which is an electron density map, or a single image of the structure. As such, it would be expected that the superposition map would contain more than one image. As shown in chapter 2 , an unweighted superposition or a superposition using a multiple vector will contain more than one image. These extra images, which could be entire images or fragments of images, will have the largest effect on the phases of the $G_{h}$. The phases as obtained from the Fourier transform of this multiple image map would give erroneous values for the phases of the $F_{h}$ if a transformation such as equation (3.2) were used. These errors, when introduced into the EG relation, or a $\Sigma_{2}$ relation, would likely cause the refinement to diverge. Thus it would be advantageous to be able to locate the additional image(s) in the superposition map and correct the $G_{h}$ for this(these) images.

Let us again consider the glide form of the compound "Elephantine". If we have a superposition map containing two images of the structure, as shown in Figure 3.3, then shifting a copy of the map by the appropriate translation $t$ should place the dashed image on the solid image. Thus the overlap of the two maps would be a maximum. similar to the 


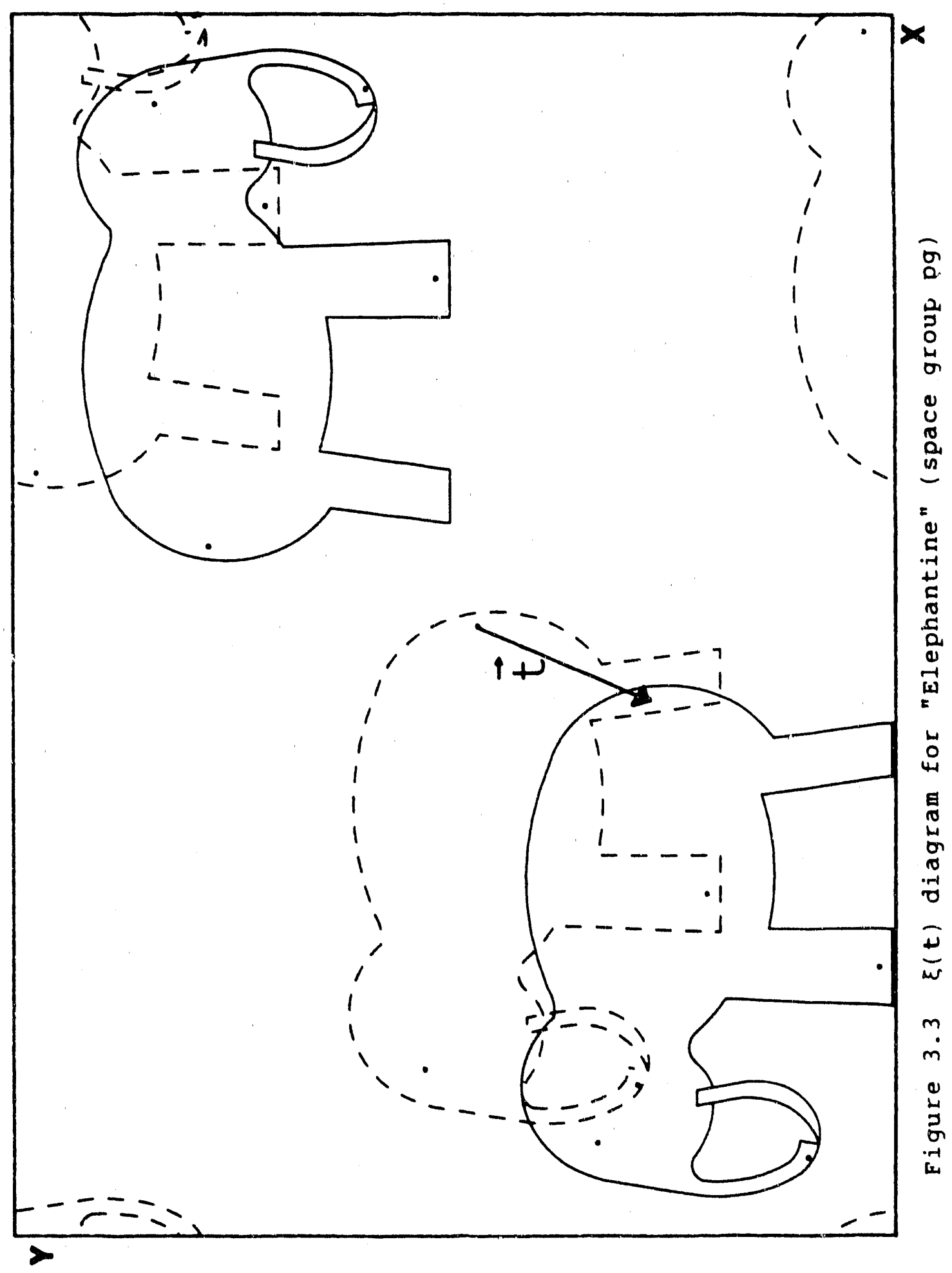


case of the $Q(\hat{s}, t)$ function, this overlap function, $\varepsilon(t)$, is given by

$$
\begin{aligned}
E(t) & =\int M(r \mid \hat{E}, 0) M(r+t \mid \hat{E}, 0) d r \\
& =\sum_{h} \sum_{k} G_{h} G_{k} \exp (-2 \pi i k \cdot t) \int \exp (-2 \pi i(h+k) \cdot r) d r \\
& =\sum_{h} G_{h} G_{-h} \exp (2 \pi i h \cdot t) \\
& =\sum_{h}\left|G_{h}\right|^{2} \exp (2 \pi i h \cdot t)
\end{aligned}
$$

One also has to be concerned that there may be an inverse image in the map. This situation is analogous to the presence of a non-inverted image and thus, the overlap is given by

$$
\xi^{\prime}(t)=\sum_{h} G_{h} G_{h} \exp (-2 \pi h \cdot t) .
$$

Once the translation for the location of an additional image has been located, the $G_{h}$ of the original map should be modified so as to remove the effect of the extra image. There are two reasonable methods of doing this. The first method would be to say that the transformed, or new, map is the sum of the original map and the map backshifted by the translation $t$. This is given by

$$
M^{\text {new }}(r)=M(r)+M(r-t) .
$$

The Fourier transform coefficient of the new map, in terms of the fourler transform coefficients of the original map, are

$$
G_{h}=G_{h}+G_{h} \exp (2 \pi i h \cdot t) .
$$


This definition has the disadvantage that if there is a peak at $r$ but not at $r$ - $t$ in the original map, there will still be a peak in the new map at $r$.

The second method would be to say that the new map is the product of the original map and the backshifted map,

$$
M^{\text {new }}(r)=M(r) M(r-t) .
$$

Using this definition, the Fourier transform coefficients of the new map are

$$
G_{h}^{e w}=\sum_{k} G_{h-k} G_{k} \exp (2 \pi i k \cdot t) .
$$

This definition removes the disadvantage found in the additive map, but it has the disadvantage of distorting the original map if the siructure is not an equal-atom case.

in the case of an unveighted superposition, the presence of the inverse image causes a small, local maximum where the global minimum should be (i.e., the location of the symmetry element) in the $S(\hat{S}, t)$ function. A modified version of $S(\hat{S}, t)$ has been developed which "mirrors" those phase differences, $\Delta(-\pi / 2 \leq \Delta \leq \pi / 2)$, that are greater than $\pi / 4$ across $\pi / 4$,

$$
S(S, t)=\frac{1}{4 \pi^{2}} \sum_{h}\left|G_{h}\right|\left|G_{h} \cdot\right| \Phi^{2}
$$

where 


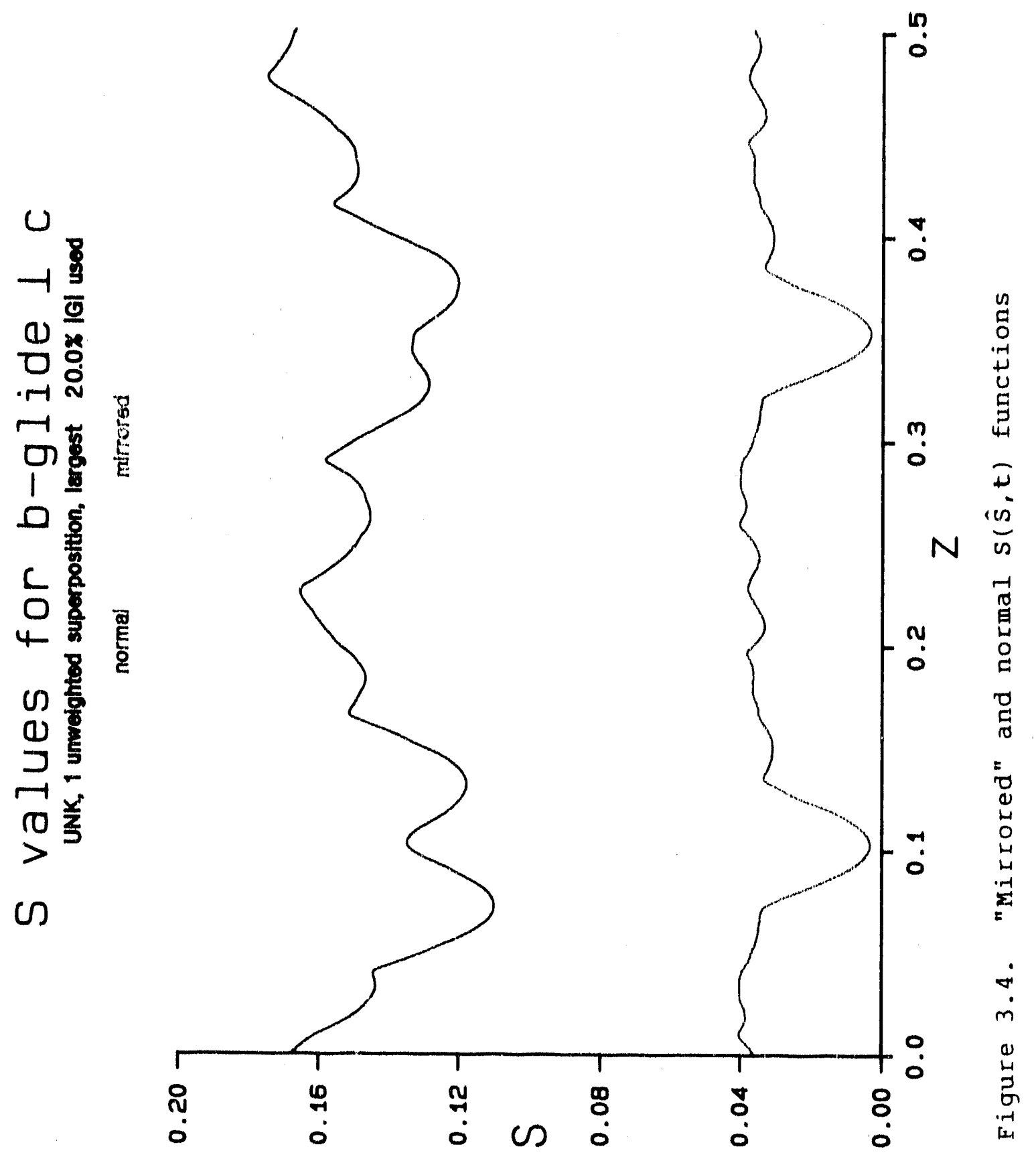




$$
\Phi=\left\{\begin{array}{l}
\frac{\pi}{2}-\Delta, \Delta>\frac{\pi}{4} \\
\Delta,-\frac{\pi}{4} \leq \Delta \leq \frac{\pi}{4}, \\
-\frac{\pi}{2}-\Delta, \Delta<\frac{\pi}{4}
\end{array},\right.
$$

and

$$
\Delta=\alpha_{h}-\alpha_{h^{\prime}}+2 \pi h \cdot(I-R) \cdot t-h \cdot T .
$$

A plot of the "mirrored" $s(\hat{S}, t)$ function and the normal $S(\hat{S}, t)$ function is shown in Figure 3.4. The "mirrored" $S(\hat{S}, t)$ function removes the local minimum at the symmetry element location, but it also produces a solution $1 / 4$ of the unit cell away from the actual solution. This is simply a result of the "mirroring" and such pseudo-solutions can be eliminated after examination of the $Q(\hat{S}, t)$ function. 
CHAPTER 4. DETAILS OF THE HYPAD PROCEDURE

Briefly, the HYPAD (HYbrid FAtterson superposition Direct methods) procedure can be broken down into the following steps:

(1) calculation of a superposition map, using the program SUPR in the CHES.CAT routine or other suitable program.

(2) GCALC - calculation of the rourier coefficients of the superposition map.

(3) LOCSYM - location of the symmetry elements in the superposition map using a reciprocal space approach

(3a) MULTIM - location of multiple images, both forward and inverse.

(3b) TRIM - removal of peaks in the superposition map using a symmetry operator and its location in the superposition map.

(4) ORSHIFT - translation of the electron density map origin to the origin of the superposition map.

(5) PHASS - direct transfer of the phases of some of the $G_{h}$ to the $E_{h}$.

(6) EGOR - refinement of the some of the other phases, via the EG relationship.

(7) FOUR - calculation of an electron density map and peak interpretation. 
The procedure, except step (1), wlll be discussed in more detail. In addition, there are several graphics programs which are available and will also be discussed. The input files that must be created by the user and that are not created by the CHES, CAT procedure use keyword-style input. These flles and their keyword options are ilsted in Appendix A. All of the plots in Chapter 5 were generated using these programs.

There are several companion programs which set up the input files for the individual steps. These programs, as well as ORSHIFT and PHASS, make extenslve use of the screen managment facility of VAX/VMS. This allows parameters to be entered using a menu-format.

HYPAD

GCALC calculates the Fourier transform coefficients of the superposition map. The program calculates the coefficients by reading in the positions and the heights of the peaks in the superposition map. The "atomic" number of the peaks, or "atom-peaks" is estimated from the height of the origin peak, and the $G_{h}$ are calculated using an expression similar to equation (2.6). The Fh are transformed into $E_{h}$ and a wilson plot is calculated, providing both an approximate least-squares scale factor and an average isotroplc thermal parameter value. A listing of 
the number and percentage of the total of both $G_{h}$ and $E_{h}$ as a function of magnitude are output. This can provido early information as to how "clean" the superposition was. The closer the $\sigma_{h}$ and the $E_{h}$ distributions agree, the likelier it is that the superposition map closely resembles the electron density map.

LOCSYM uses the theory presented in Chapter 3 to determine the location and/or existence of symmetry elements in Patterson superposition maps. For symmetry planes, a preliminary test can be made to determine the plausibility of the existence of a symmetry plane. This is most useful when the extinction conditions do not clearly indicate the presence of a symmetry plane.

The translation cholces are determined by first. calculating $Q(\hat{s}, t)$. Then for those translations that appear most promising, $s(\hat{S}, t)$ is calculated and the figure-of-merit is used to determine the "best translation cholce. The translations for the symmetry elements are then compared for consistency based on the requirements for forming a group 1.e., in class mmm, a 2-fold in $x$ operating on a 2-fold in $y$ must give a 2 -fold in $z$ ), and those symmetry eloments that show consistency are indicated.

For one- and two-dimensional translations, it takes a small amount of computer time to calculate the entire spectrum of translations (within 0.0001 for one dimension, 0.01 for two dimensions). However, it requires a larger 
amount of computer timo to calculate the threo dimenalonal translation spectrum. One-dimenstonal operators take about a minute of CPU time, two-dimenisonal operators take 1-4 minutes, and three-dimensional operators take 5 mio minutos. lof course, these times depend upon how many datia are being using to do the calculations, larger data sets require longer times than smaller unes.) Experience has shown that inclusion of $G_{h}$ having magnitudes less than undty do not make a qualitative difference in the appearance of the $Q(\hat{S}, t)$ and $s(\hat{S}, t)$ functiong.

In practice, the $S(\hat{S}, t)$ function is usualiy calculated according to equation (3.9). However, the uner is given the option of using an alternate weighting function, $\left|E_{h}\right|\left|G_{h}\right|\left|G_{h} \cdot\right|$

MULTIM uses the theory presented fin Chapter 3 to locate translations between multiple images. For contered colls, one of the solutions will be the centering operation. For example, a C-centered cell wlll produce values of $E(t)$ that are approximately unity for the translations $(0,0,0)$ and $\left(b_{2}, b_{2}, 0\right)$.

TRIM reduces the list of peaks in the superposition map based on the presence of symmetry related peaks. This is an optional procedure, which I have found is very useful for eliminating extraneous peaks in the map. The symmetry operator to be used and its translation (as given by LOCSYM) are input and the program then searches the poaks 11 st and 
oliminates all peaks for which a match cannot be found. The range of peaks to be considered and the tolerance, in $A$, for finding a match are input by the user.

After execution of TRIM, GCALC and LOCSYM should be rerun, llaing the now poaks list. Thla will provide now phases, which should be better, for use in the rest of the package.

ORSHIFT modifies the phases of the $G_{h}$ so that they are referenced to the electron density map ordgin, according to equation (3.2). Once the phases have been modifled, the symmetry-related $G_{h}$ are averaged. For centrosymmetric structures, the phases of the $G_{h}$ are constralned to be 0 or $\pi$.

PHASS sets the phases of some of the larger $E_{h}$ by difectly transferring the phase of the corresponding $G_{h}$. The criterion for phase transferability is glven by wang 37 . At this step, the user is given the option of creating a set of Fourier coefficients suitable for use with Four (step 7). The coefficients are constructed by combining the magnitude of the $E_{h}$ with the phase of the $G_{h}$. Elther the complete set of reflections or the set containing nnly those reflections for which phases can be transferred can be created. For simple centrosymmetric structures, direct transfer of all of the phases and then calculation of an electron density map can often result in a solution. 
EGOR tefines the phasels of the Eh using the EG relationship. The welghted tangent equation, as given by wang 37 , is used for the refinement. In centrosymmetric space groups, the phases of the $\mathbb{E}_{\mathrm{h}}$ are constralned to be 0 or $\pi$. Only those $\mathbb{E}_{h}$ with a magnitude greater than or equal to a minimum magnitude, aupplited by the user, are refined. The user can set the maximum number of cycles of refinement, but the refinement will automatically terminate whon no new phases are added or modified. A phase is added (or modified) when the weight for the new phase is greater than 1. - $n / 100$ (or the current welght), where $n$ is the cycle of iteration.

TOUR is actually a series of programs that calculate and interpret an electron density map. These routines were taken from the CHES.CAT procedure and modified to accept keyword input.

\section{Graphics Programs}

There are six graphics programs avallable in HYPAD. These are called QPLANES, QSPLOT, QROTLINE, QDATA, 2DDRAW, and 3DDRAW. Two of the programs, 2DDRAW and 3DDRAW, are interactive plotting routines that make use of the DI 3000 graphics routines.

2DDRAW is a two-dimensional contouring program. The user is given control over both the region to be plotted and 
the range of contours. Individual contour lines are drawn every ten percent of the contouring range. Depending upon the device used, the contours are drawn elther in oolor or with different linestyles.

3DDRAW is a contouring program that draws threedimensional "fishnet" profections. The user has more limited control over the appearance of the plot, and with because of the addition of a third dimension, all contour lines are drawn with the same color and linestyle.

QPLANES generates a "plot" containing the $Q(\hat{s}, t)$ values for all space group symmetry planes perpendicular to the axis indicated. All five symmetry plane types (mirror, glides in the other two directions, n-glide, and d-glide) are plotted on the same graph. Plots for more than one axls can be generated in the same run. The "plot" is written out to a flle as a sertes of HP graphics language commands and can be plotted on an HP plotter.

QROTLINE generates a plot of the $Q(\hat{s}, t)$ values for a rotation symmetry operator, where one of the translation directions is fixed at some value. As in QPLANES, the plot is written out to a flle as a series of HP graphics language commands.

QSPLOT generates a plot containing the $Q(\hat{s}, t)$ and $S(\hat{S}, t)$ values for a symetry plane. The $Q(\hat{s}, t)$ values are plotted in red and the $S(\hat{S}, t)$ values are plotted in green. The calculation of the $s(\hat{s}, t)$ values is the most time consuming 
partt off the pirogram"s exerution. In order to use enough datta sio that am accuratte Inemesemitation of the $s,(\vec{S}, t)$ ffumction is abttaimed amd sitill use a rellatively small amount

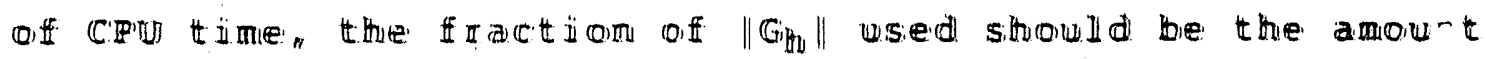

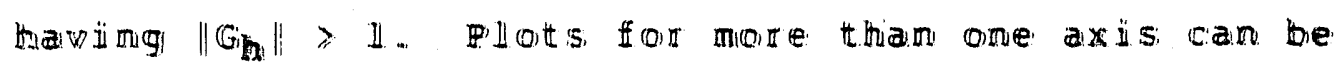
glemeratted in the same rim.

QDATA gemerates $Q\left(\hat{S}_{n} t\right)$ or $S\left(\hat{S}_{n} t\right)$ vallues for a symmetry operater amid withes the data to a fille. This is the program thiat ome would wse in comjunction with the imteractive

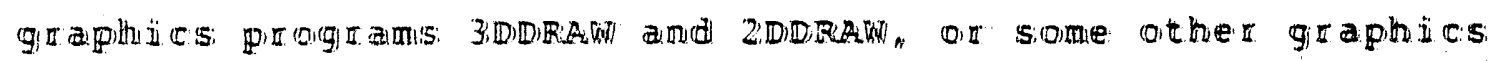

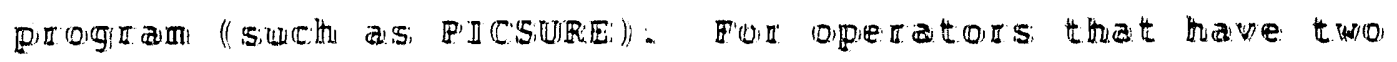

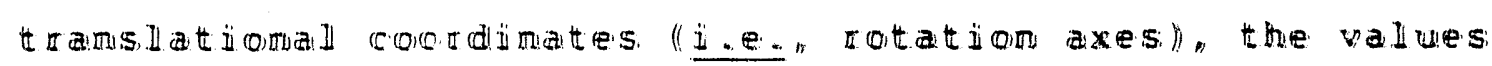
are callewated for a square grid thaving proints every . ol in

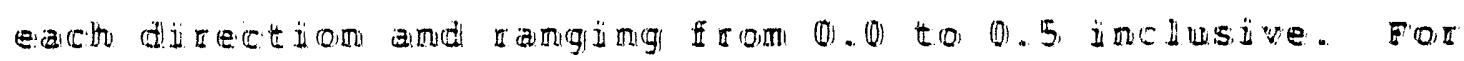

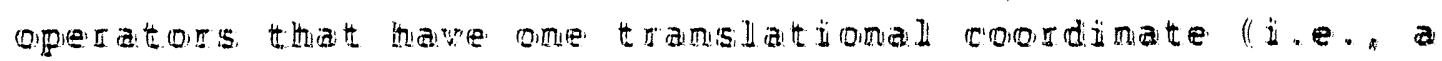

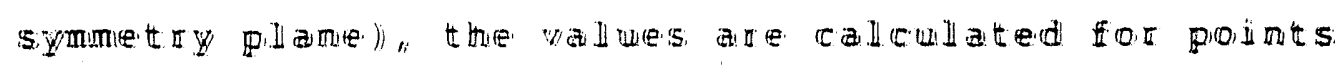

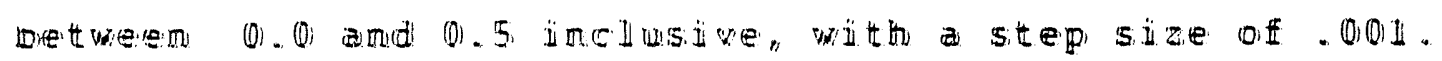


CHAPTER 5. APPLICATION OF HYPAD TO THE SOLUTION OF KNOWN AND UNKNOWN STRUCTURES

Any automated Patterson-based technique should be able to. satisfy certain minimum requirements. These requirements showld include the ability to solve structures, and applicability to structures of any symmetry type, both centrosymetric and non-centrosymmetric.

The heavy atom method for the determination of phases is based upon the assumption that the heavy atom(s) present in the structure make up a significant portion of the overall electron density. A general rule of thumb is that if $z_{H}{ }^{2}$ > $\mu \Sigma z_{\mathbb{L}}{ }^{2}$ holds, where $z_{H}$ is the atomic number of the heavy atom. $Z_{\mathbb{L}}$ are the atomic numbers of the light atoms, and $\mu$ is in the range of to 1 ; then the heavy atom method is likely to succeed. This condition is typically met by organometallic compounds since they usually contain a central metal atom surrounded by an organic ligand. However, there are many organometallics that fail to fall into this category. In these cases, phase predictions based solely upon the metal atom are usually erroneous and subsequent electron density maps are of little use. Clearly, these cases require the positions of several atoms be determined and not just the position of the metal atom. 
HYPAD Solution of $\mathrm{Cr}(\mathrm{CO})_{3} \cdot \mathrm{C}_{6} \mathrm{H}_{6}$

This compound represents a very simple organo-metallic, containing one metal atom per molecule. It also meets the criterion for the heavy-atom method easily $(\mu=1.1)$. This makes it a very attractive first test for the reciprocal space methods. The HYPAD solution of this molecule turned out to be extremely straightforward.

An ORTEP drawing of $\mathrm{Cr}(\mathrm{CO})_{3} \cdot \mathrm{C}_{6} \mathrm{H}_{6}$ (BCT) is given in Figure 5.1. Extinction conditions on the observed intensities failed to uniquely determine the space group. but did indicate the presence of a $22_{1}$-screw axis parallel to b. Since the compound crystallizes in the monoclinic system, there are two possible space group choices, P21 (non-centrosymmetric) and $\mathrm{P}_{1} / \mathrm{m}$ (centrosymmetric).

A vector corresponding to a $C r-C$ vector was used as the shift vector for a weighted superposition. The rourier transform coefficients of the superposition map were determined and the $Q(\hat{S}, t)$ and $S(\hat{S}, t)$ functions were calculated for the mirror plane, the $2_{1}$-screw axis and the inversion operator. Figures 5.2 and 5.3 show plots of the $Q(j, t)$ function for symmetry planes perpendicular to $b$ and the $2{ }_{1}$-screw axis parallel to $b$, respectively. The contour layers in figure 5.3 are color-coded, with the contours every 10 percent of the range from 0 to the maximum $Q(\hat{S}, t)$ value on the map. The colors and their percentage levels 
0

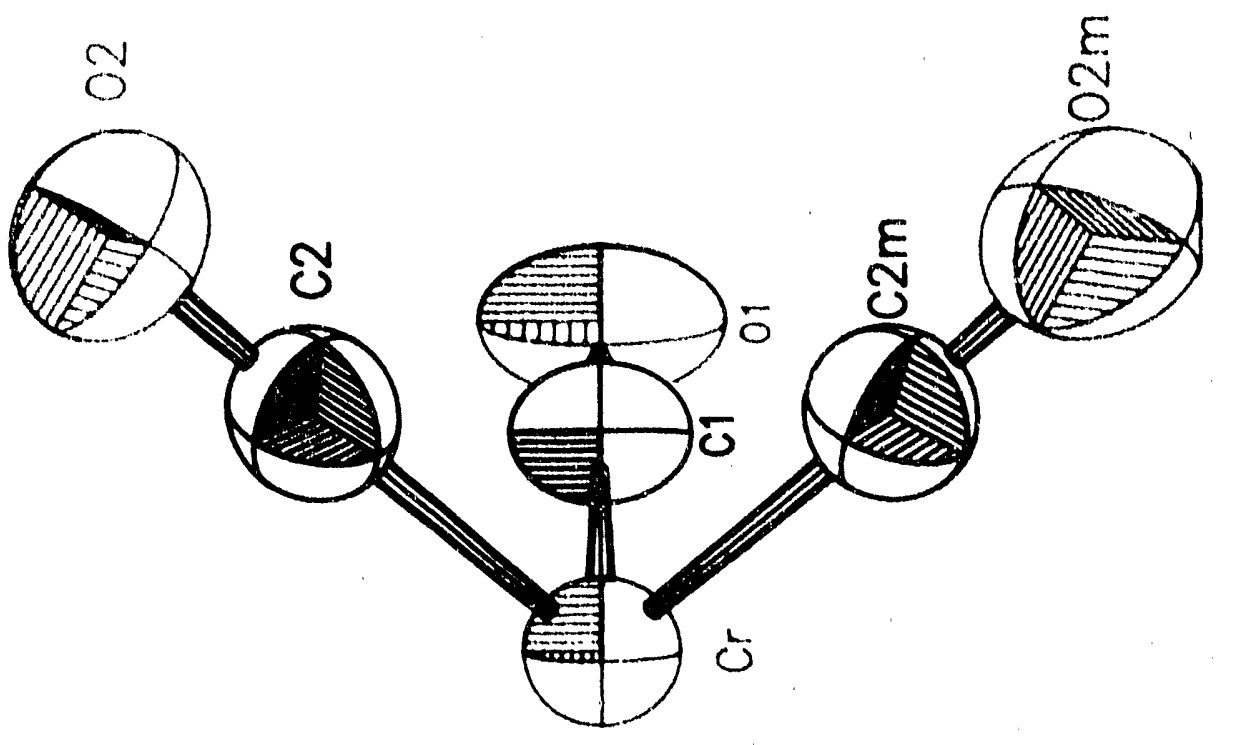

U

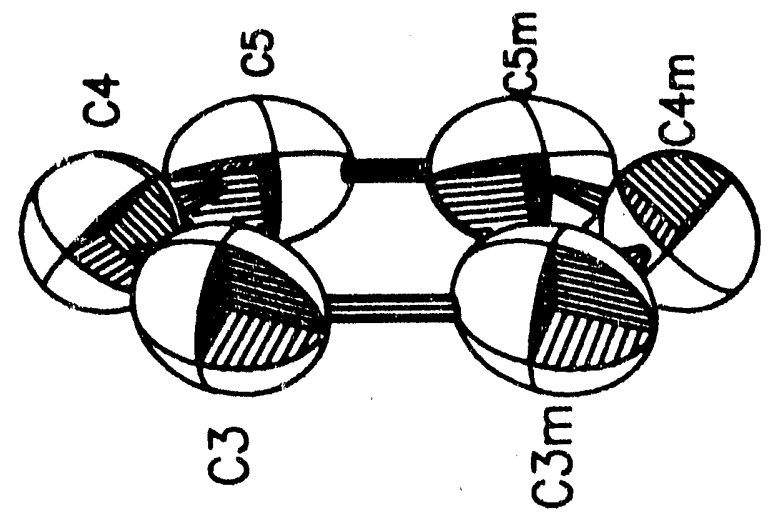




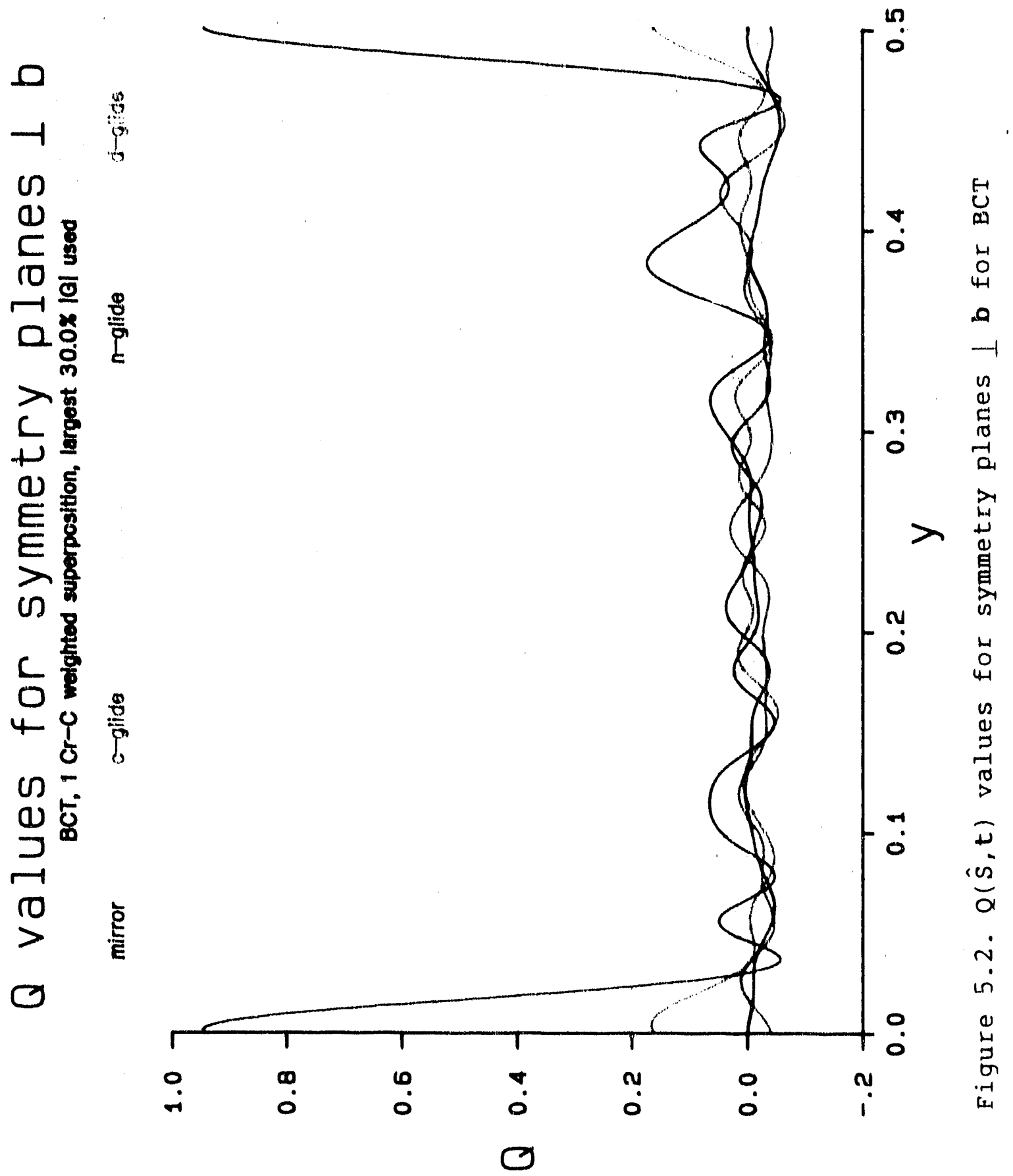




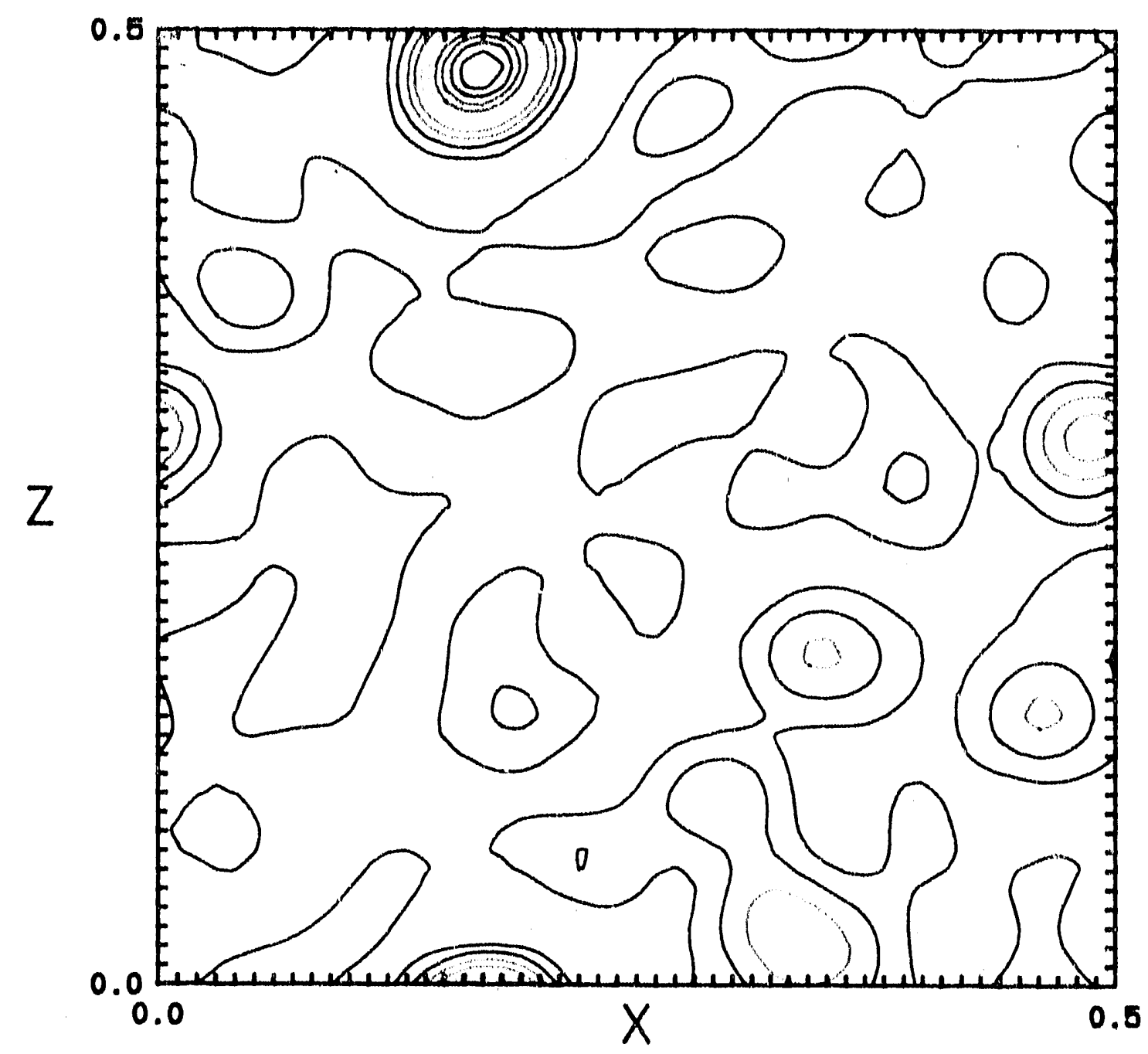

Figure 5.3. $Q(\hat{S}, t)$ values for $21^{-s c r e w}$ axis || b for $\mathrm{BCT}$ 
Table 5.1. Symmetry Operator Locations for $\mathrm{Cr}(\mathrm{CO}) 3^{\circ} \mathrm{C}_{6} \mathrm{H}_{6}$

\begin{tabular}{ccccc}
\hline symmetry operator & $t^{a}$ & $Q(\hat{s}, t)$ & $s(\hat{s}, t)$ \\
\hline$-x, 1 / 2+y,-z$ & $(0.1681,0.0,0.4784)$ & 0.8407 & 0.0168 \\
$x,-y, z$ & $(0.0,0.0009,0.0)$ & 0.9544 & 0.0044 \\
$-x,-y,-z$ & $(0.1681,0.2515,0.4782)$ & 0.7902 & 0.0234 \\
\hline
\end{tabular}

aranslations are given in fractions of a unit cell.

are: red $(0 \%, 10 \%)$, orange $(20 \%, 30 \%)$, yellow $(40 \%, 50 \%)$, green $(60 \%, 70 \%)$, and blue $(80 \%, 90 \%)$. Consistent translations, listed in Table 5.1, were found for all three operators, indicating that the space group was $\mathrm{P} 21 / \mathrm{m}$. It turns out that the Cr atom lies on the mirror plane (as does one of the carbonyls).

Therefore, the large peak at the origin in the $Q(\hat{S}, t)$ function of the mirror in figure 5.2 is from the $\mathrm{Cr}$ image. There is a smaller peak at 0.38 which is the location of the mirror plane in the $C$ image. It is interesting to note that the ratio of the values of the $Q(\hat{s}, t)$ function at these two points is approximately the value of the weight applied to the superposition. There is only one large peak in the plot for the $21^{-s c r e w}$ axis, and it is the location of the $2_{1}$ screw axis in the Cr image.

Since the origin in the space group lies on the inversion center, the $G_{h}$ were transformed using the inversion center location, according to equation (3.2). The 


\section{3}

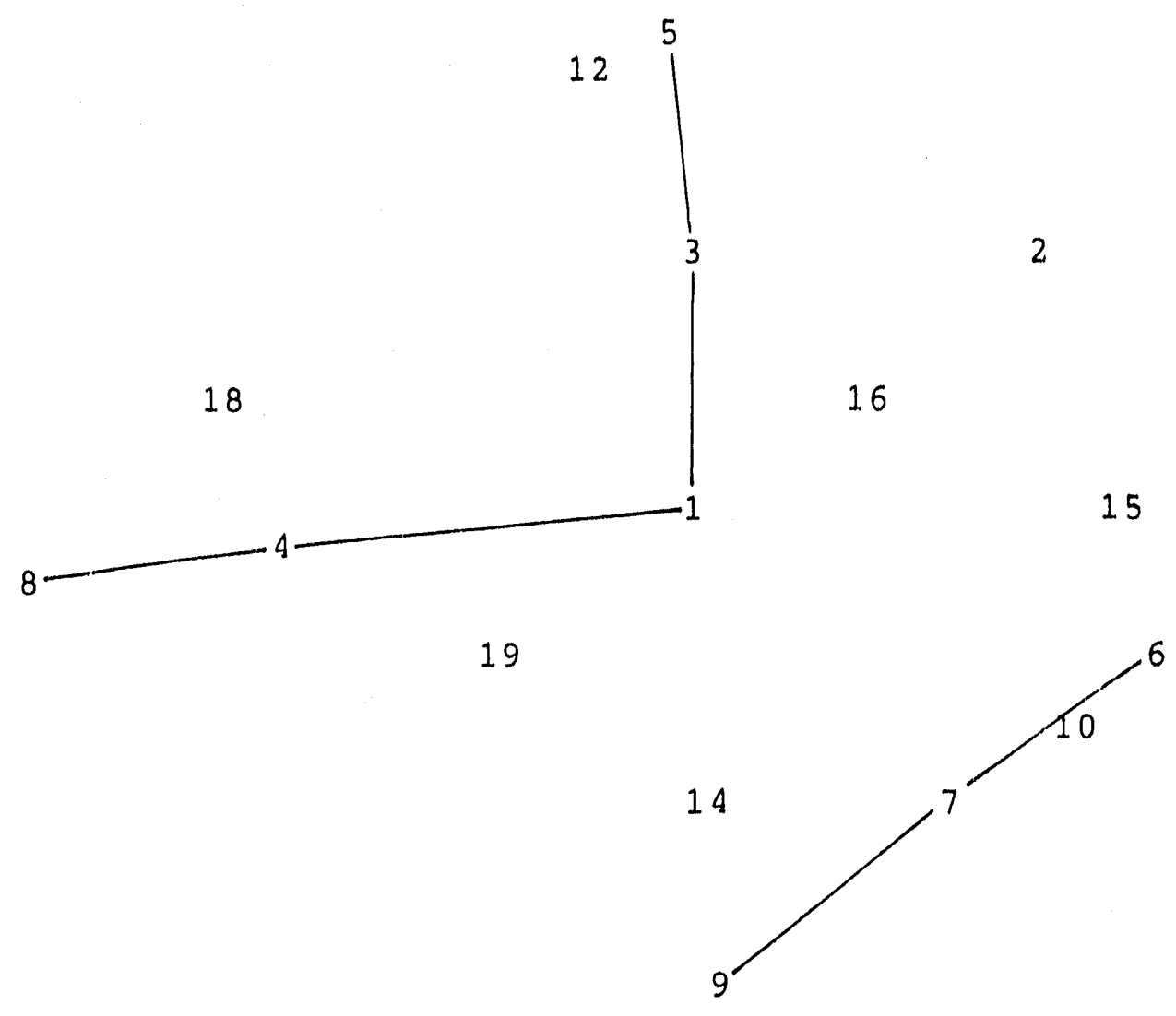

Figure 5.4. HYPAD-generated projection of $\mathrm{Cr}(\mathrm{CO}) 3^{\circ} \mathrm{C}_{6} \mathrm{H}_{6}$ 
Table 5.2. HYPAD Atomic Coordinatesa $\left(\times 10^{4}\right)$ for $\mathrm{Cr}(\mathrm{CO})_{3} \cdot \mathrm{C}_{6} \mathrm{H}_{6}$

\begin{tabular}{crrrrr}
\hline atom & $\#$ & pk.ht. & $x$ & $y$ & $z$ \\
\hline Cr & 1 & 505 & 1692 & 2500 & 4755 \\
01 & 8 & 90 & -1971 & 2500 & 1050 \\
02 & 5 & 96 & 4140 & 642 & 2700 \\
C1 & 4 & 108 & -520 & 2500 & 2398 \\
C2 & 3 & 11 & 3131 & 1396 & 3572 \\
C3 & 6 & 96 & 3234 & 1854 & 7962 \\
C4 & 7 & 95 & 1281 & 1260 & 7270 \\
C5 & 9 & 81 & -739 & 1852 & 6534 \\
\hline
\end{tabular}
cell.

atomic coordinates are given as fractions of the unit

Table 5.3. Refined Atomic Coordinatesa $\left(\times 10^{4}\right)$ for $\mathrm{Cr}(\mathrm{CO})_{3} \cdot \mathrm{C}_{6} \mathrm{H}_{6}$

\begin{tabular}{cccc}
\hline atom & $x$ & $y$ & $z$ \\
\hline $\mathrm{Cr}$ & $1682(1)^{b}$ & 2500 & $4755(1)$ \\
01 & $-1917(7)$ & 2500 & $1005(6)$ \\
02 & $4094(6)$ & $668(3)$ & $2744(4)$ \\
$\mathrm{C} 1$ & $-523(9)$ & 2500 & $2446(7)$ \\
$\mathrm{C} 2$ & $3167(6)$ & $1359(3)$ & $3548(5)$ \\
$\mathrm{C} 3$ & $3202(6)$ & $1868(3)$ & $7967(5)$ \\
$\mathrm{C} 4$ & $1215(7)$ & $1231(3)$ & $7271(5)$ \\
$\mathrm{C} 5$ & $-753(6)$ & $1858(4)$ & $6585(6)$ \\
\hline
\end{tabular}
cell.

atomic coordinates are given as fractions of the unit

bestimated standard deviations for the refined coordinates are given in parentheses for the least significant digit. 
Table 5.4. Comparative bond distances for $\mathrm{Cr}(\mathrm{CO}){ }_{3} \cdot \mathrm{C}_{6} \mathrm{H}_{6}$

\begin{tabular}{cccc}
\hline atoms & $\operatorname{refined}(A)$ & HYPAD $(A)$ & $\Delta(A)$ \\
\hline$C r-C 1$ & $1.831(5)$ & 1.85 & 0.02 \\
$C r-C 2$ & $1.833(5)$ & 1.78 & $\cdots .05$ \\
$C r-C 3$ & $2.231(3)$ & 2.25 & 0.02 \\
$C r-C 4$ & $2.218(4)$ & 2.20 & -.02 \\
$C r-C 5$ & $2.207(4)$ & 2.20 & -.01 \\
$C 1-01$ & $1.144(6)$ & 1.13 & -.01 \\
$C 2-02$ & $1.143(4)$ & 1.25 & 0.09 \\
$C 3-C 4$ & $1.405(5)$ & 1.37 & -.03 \\
$C 4-C 5$ & $1.389(5)$ & 1.40 & 0.01 \\
\hline
\end{tabular}

aEstimated standard deviations for the refined distances are given in parentheses for the least significant digit.

Table 5.5. Comparative bond angles for $\mathrm{Cr}(\mathrm{CO}){ }^{\cdot} \mathrm{C}_{6} \mathrm{H}_{6}$

\begin{tabular}{cccr}
\hline atoms & refined $\left({ }^{\circ}\right)$ & HYPAD $\left(0^{\circ}\right)$ & \multicolumn{1}{c}{$\Delta\left(0^{\circ}\right)$} \\
\hline$C 2-C r-C 1$ & $89.07(16) a$ & 89.0 & -.1 \\
$C 2-C r-C 2 m$ & $86.75(21)$ & 86.7 & 0.0 \\
$C r-C 1-01$ & $179.26(46)$ & 175.2 & -3.9 \\
$C r-C 2-02$ & $179.30(30)$ & 178.4 & -.9 \\
$C 3-C 4-C 5$ & $120.09(34)$ & 123.5 & 3.4 \\
$C 4-C 3-C 3 m$ & $120.03(21)$ & 118.7 & -1.3 \\
$C 4-C 5-C 5 m$ & $119.87(23)$ & 117.8 & -2.1 \\
\hline
\end{tabular}

aEstimated standard deviations for the refined angles are given in parentheses for the least significant digits. 
phases of 75 of the largest Eh, representing 11.48 of the

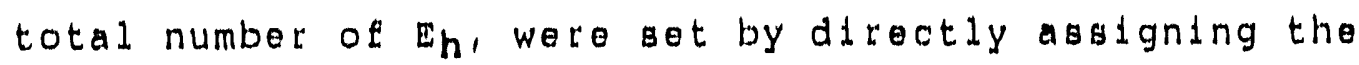
phases of the corresponding $G_{h}$. The $\mathbb{E}_{h}$ were then refined using the EG relationship and an electron density map was calculated. Figure 5.4 showa a profection of the olectron density map onto the least-squares plane as generated by HYPAD, (The bonds in this projection were drawn in by hand.) The complete molecule is clearly identifiable from this picture. The HYPAD atomic positions, along with the peak lumber and peak helghts are glven in Table 5.2 and the refined atomic positions are given in Table 5.3. All of the unique non-hydrogen atoms are found in the top 9 peaks on the map. Tables 5.4 and 5.5 compare the reflined and HYPADgenerated bond distances and bond angles for this compound. All of the bond distances in the HYPAD molecule are within $0.1 \AA$ of the refined distances and the HYPAD bond angles are within $4^{\circ}$ of the refined angles.

It is encouraging to note that even with incomplete initial knowledge of the space group, this structure can be solved with very little user input, and that the correct space group is obtained duling the procedure.

HYPAD Solution of FeP $\mathrm{OC}_{32} \mathrm{H}_{31} \mathrm{I}$

This is a slgnificantly more complicated compound, and was the first acentric structure studied using HYPAD. 
Crystals of the compound wero obtained from John Nelion's group (Department of Chemdatry, Undveradty of Nevada-Reno). It was orlgimaliy solved by another member of our group, Cathy Day, only aftor a very todious patterson analysis. As ahown in $\mathrm{rigure} 5.5, \mathrm{FeP}_{2} \mathrm{OC}_{32} \mathrm{H}_{31} \mathrm{I}$ (VII) is a very complex molecule, the type that it would be hoped that HYPAD would bo useful in solving.

This compound crystallizes in the orthorhombla system. The extinction conditions falled to uniquely determine the space group, but did indicate the presence of a c-gldde plane perpendicular to and a n-gldde plane perpendicular to c. This leaves two possible space group cholcess pod n (non-centrosymmetric) and Pemn (centrosymmetric). Direct methods falled to provide a solution in elther of the posatble space groups.

A vector corresponding to a pe-p bond distance was chosen as the shift vector for a weighted superpogition. The Fourler transform coefficients were determined and tho $Q(\hat{S}, t)$ and $S(\hat{S}, t)$ functions for all of the possible symmetry Elements in the two space group cholces were calculated. The best translations, and their corresponding $Q(\hat{s}, t)$ and $S(\hat{s}, t)$ values are listed in Table 5.6. Consistent translations were found for the c-glide plane perpendicular to a, the 21-screw axis parallel to $b$ and the n-glide plane perpendicular to $c$, indicating that the space group was Pc2 1 . Plots of the $Q(\hat{s}, t)$ function for symmetry planes 
(D)

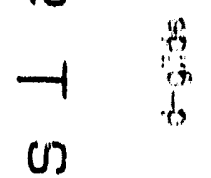

도욜

C 8

로용

C

4

$\omega-$

(1)

כ

$>\quad$ 点

(3)

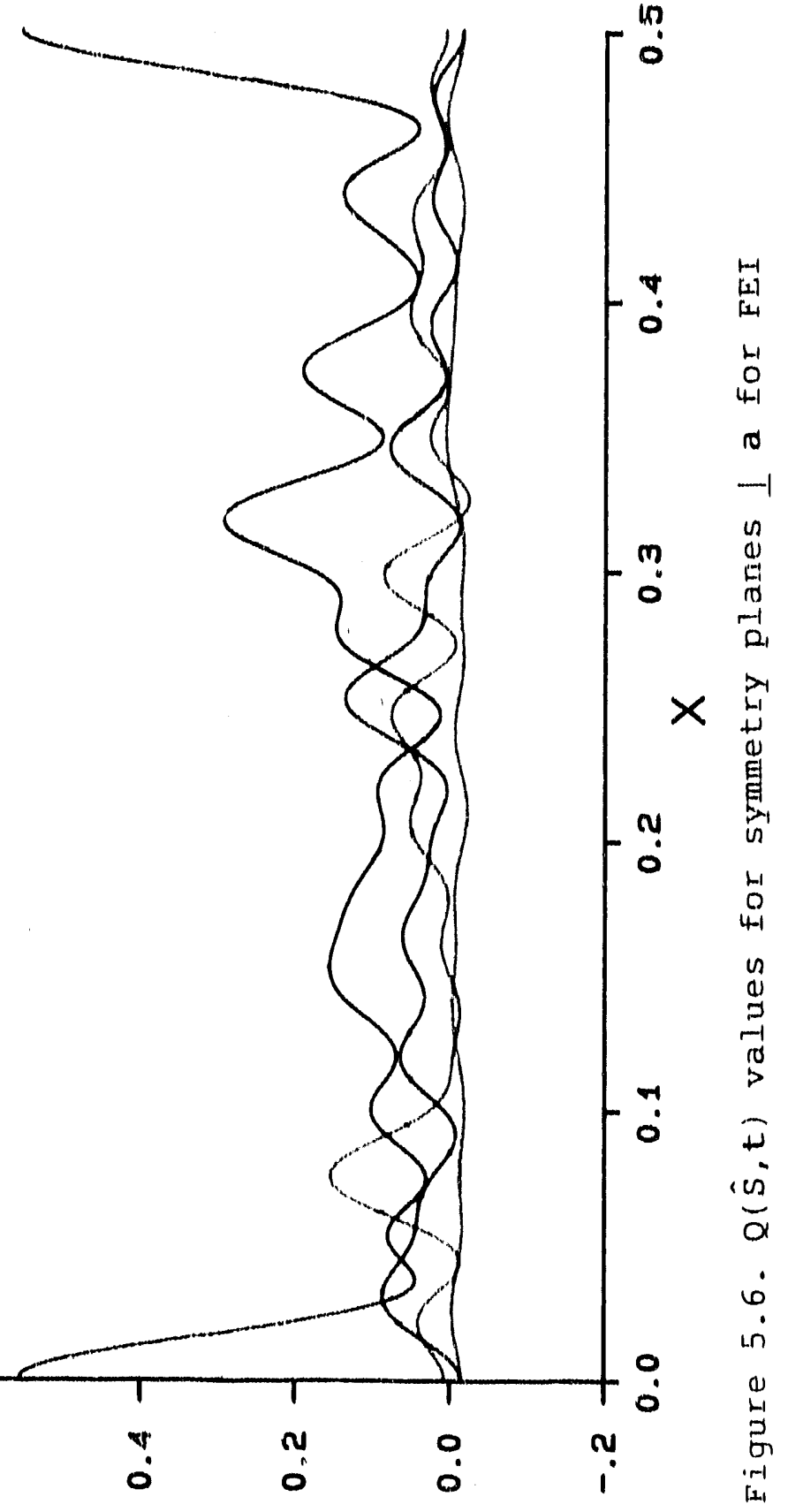

(3) 


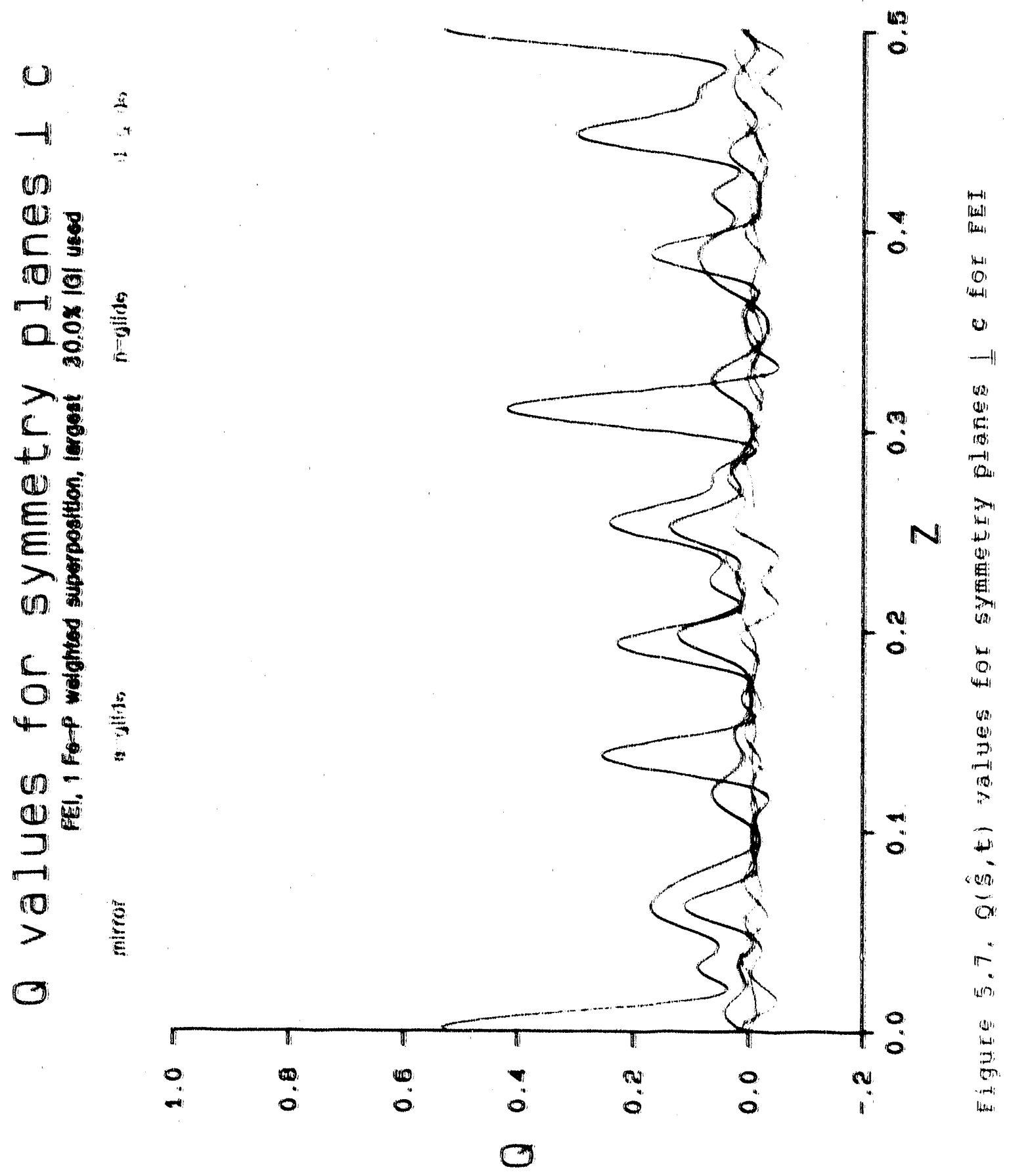




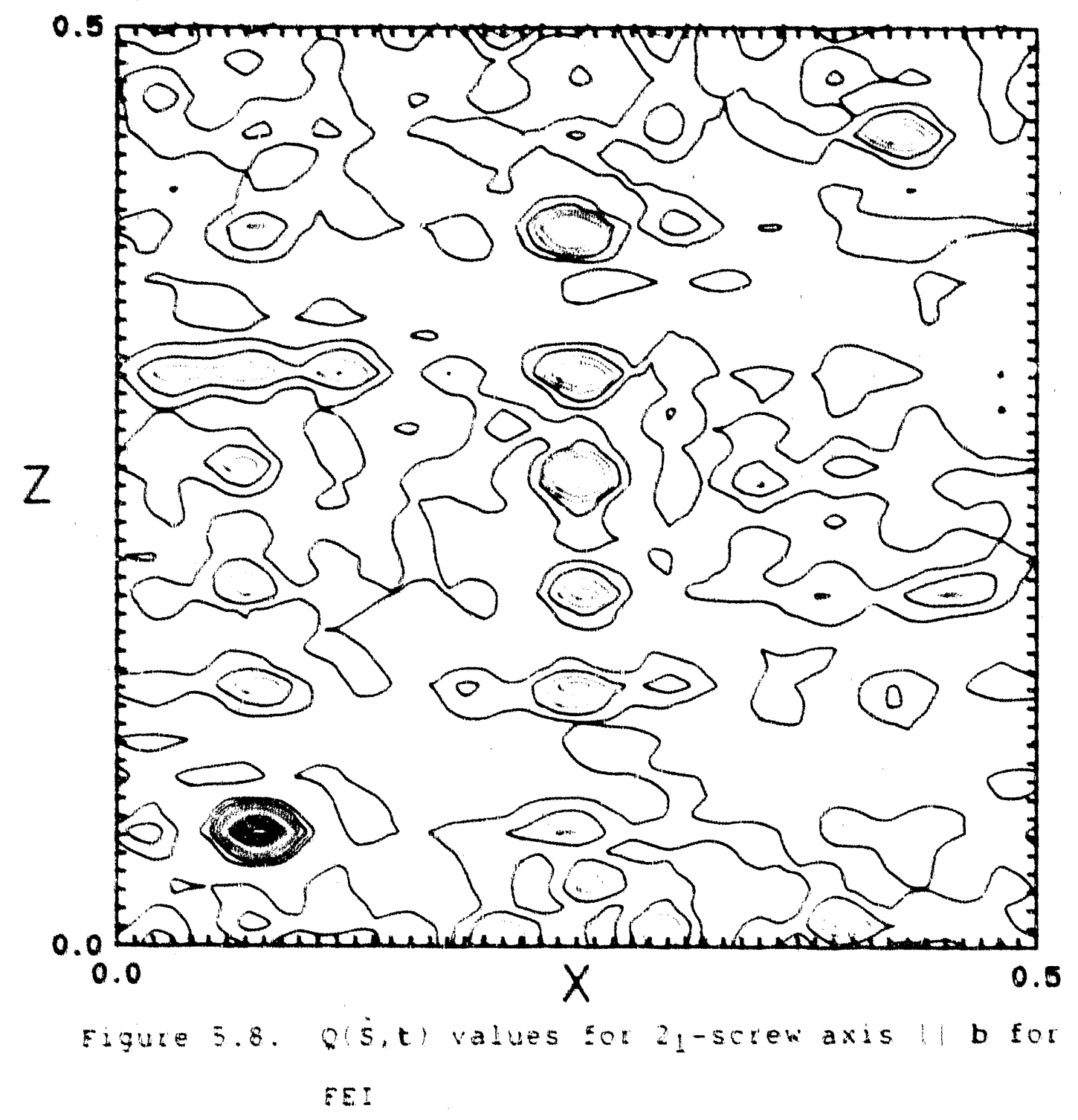


Table 5.6. Symmetry operator Locations for FeP $\mathrm{OC}_{32} \mathrm{H}_{31} \mathrm{I}$

\begin{tabular}{ccccc}
\hline symmetry operator & $t^{a}$ & $Q(\hat{s}, t)$ & $s(\hat{s}, t)$ \\
$x+12,-y,-z$ & $(0.0,0.2548,0.3106)$ & 0.3273 & 0.0888 \\
$-x, y+1,2,-z$ & $(0.0762,0.0,0.0604)$ & 0.4191 & 0.0761 \\
$-x,-y, z+1,2$ & $(0.3287,0,0046,0.0)$ & 0.3467 & 0.0874 \\
$-x, y, z+1 / 2$ & $(0.3260,0.0,0.0)$ & 0.4967 & 0.0703 \\
$x,-y, z$ & $(0.0,0.1885,0.0)$ & 0.3130 & 0.0919 \\
$x+1 / 2, y+1 / 2,-z$ & $(0.0,0.0,0.3104)$ & 0.4274 & 0.0749 \\
$-x, y,-z$ & $(0.4100,0.4845,0.4745)$ & 0.8301 & 0.0203 \\
\hline
\end{tabular}

arranslations are given in fractions of a unit cell.

perpendicular to a and $c$ are shown in Figures 5.6 and 5.7 , respectively, and for the $2_{1}$-screw axis parallel to $b$ in Figure 5.8 .

There is a sizable peak in the $Q(\hat{S}, t)$ function for the c-glide plane at approximately 0.32, which corresponds to the location of the glide plane in the Fe image. It turns out that the $p$ atom used in the superposition vector lies almost on the glide plane. Thus, the peak at the origin of the $Q(\hat{s}, t)$ function indicates the location of the glide plane in the $P$ image. As in the case of $\mathrm{Cr}(\mathrm{CO})_{3} \cdot \mathrm{C}_{6} \mathrm{H}_{6}$, the ratio of the $Q(\dot{s}, t)$ values for these peaks is close to the weight used in the superposition.

The $Q(\hat{S}, t)$ function for the $n-g l i d e$ plane perpendicular to c has sevelal peaks, the largest of which, at approximately 0.31 , is the location of the glide plane in the fe image. The next highest peak is the location of the 
glide plane in the $P$ image. Again, the ratio of the $Q(\hat{S}, t)$ values for these two peaks is approximately the weight used for the superposition.

There is one peak in the $Q(\hat{S}, t)$ function for the $2_{1}$ screw axis parallel to $b$, at approximately $x=0.07, z=$ 0.06 , which is obviously much higher than the other peaks. This is the location of the screw axis in the Fe image. The next highest peak, at approximately $x=0.25, z=0.39$, is location of the $2_{1}$-screw axis in the $P$ image.

Another interesting feature in this map is the line of peaks having $x=0.25$. If this line of the $21^{-s c r e w}$ axis map is plotted on the same diagram as the $n$-glide plane perpendicular to $c$, as in Figure 5.9, an interesting feature is observed. There is a very strong correlation between the peaks in the two curves. Essentially every place that there is a peak in one curve, there is a peak in the other curve.

As can be seen from the $Q(\hat{S}, t)$ functions for the symmetry planes, there is still quite a bit of pseudosymmetry remaining from the patterson map in the superposition map. This pseudo-symmetry can interact with the actual symmetry of the unit cell to produce still more pseudo-symmetry. However, the general location of this "interaction" pseudo-symmetry can be predicted.

Let us assume that there is still pseudo-symmetry remaining from the patterson map in the form of a mirror plane perpendicular to a. In the patterson map, this mirror 

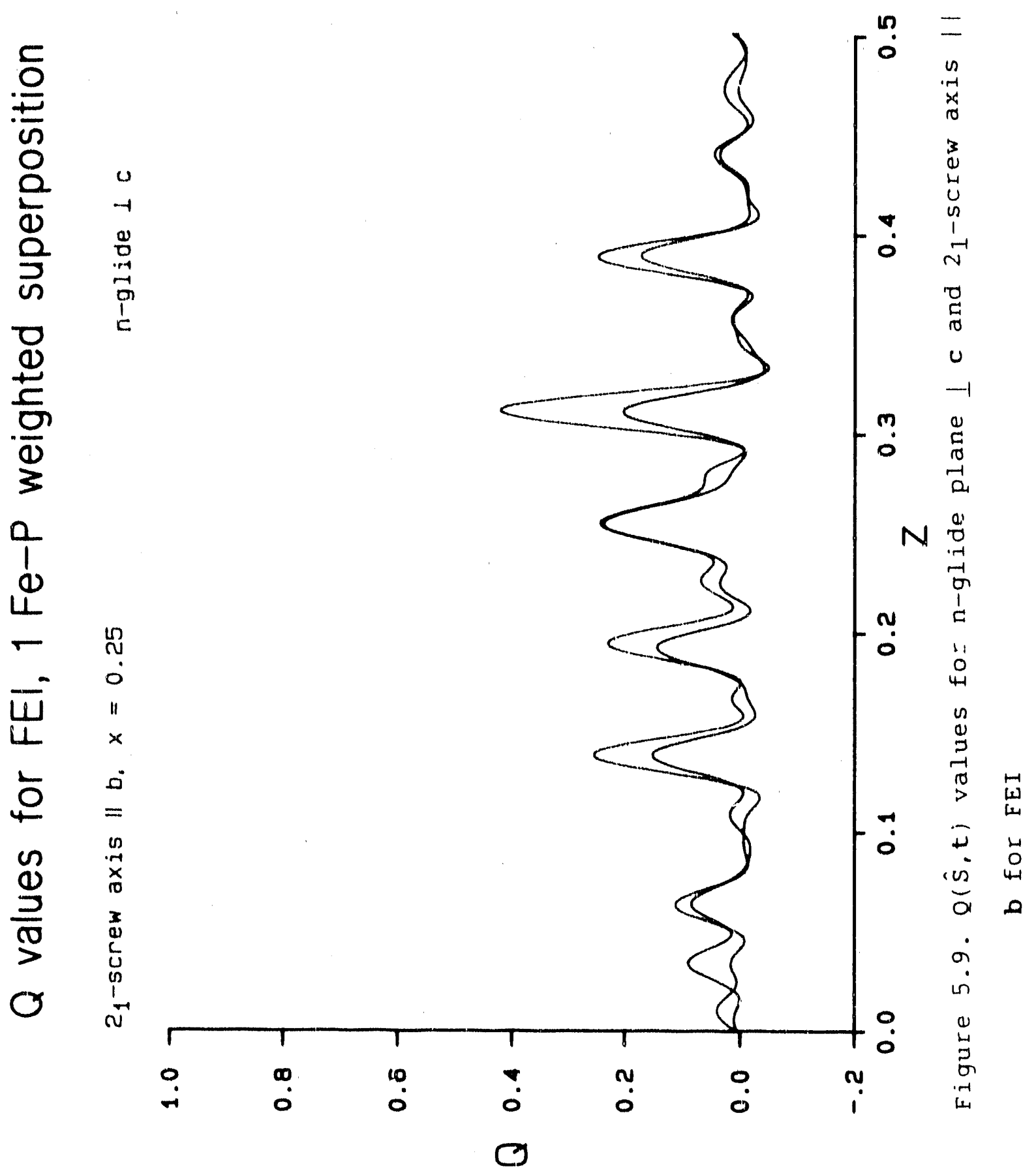
plane lies at $x=0$, and so a point at $x, y, z$ in the superposition map generates at pseudo-point at $-x, y, z$. Because of the $n-g l i d e$ plane perpendicular to c, located at $t_{z}$, a peak at $x, y, z$ will have a symmetry-related peak at $1 / 2+x, 1 / 2+y, 2 t_{z}-z$. This symmetry-related peak will generate, by the pseudo-mirror, a pseudo-peak at $1 / 2-$ $x, 1 / 2+y, 2 t z^{-z}$. The peak at $x, y, z$ is "related" to the pseudo-peak at $1 / 2-x, 1 / 2+y, 2 t_{z}-z$ by a pseudo-2 $1-s c r e w$ axis parallel to b. This pseudo-2 1 -screw axis is located at $\left(0.25,0.0, t_{z}\right)$. Thus, the two peaks in the $Q(\hat{s}, \hat{E})$ function at $(0.25,0.0,0.14)$ and $(0.25,0.0,0.31)$ axe the locations of pseudo-symmetry elements caused by the n-glide plane in the $P$ image and the $F e$ image, respectively. The $n-g l i d e s$ should also produce pseudo-2 1 -screw axes parallel to a at $(0.0,0.25,0.31)$ and $(0.0,0.25,0.14)$. Examination of Table 5.6 shows that the "best" translation for the $2_{1}$-screw axis is at $(0.0,0.255,0.311)$. There is also a peak at $(0.0,0.253,0.138)$.

Using similar reasoning, the c-glide plane perpendicular to a will produce pseudo-2 1 -screw axes parallel to $c$ at $\left(t_{x}\right.$, $0.0,0.0)$. According to Table 5.6, the c-glide plane is located at $(0.326,0.0,0.0)$. This should produce a pseudo$21^{-s c r e w}$ axis at approximately $(0.326,0.0,0.0)$ and examination of Table 5.6 shows that the "best" translation for the $21^{\text {-screw }}$ axis is at $(0.329,0.005,0.0)$. The cglide plane in the $P$ image is located at $(0.0,0.0,0.0)$ 
which should produce a pseudo-21-9crew axis at $(0.0,0.0$, $0.0)$. There is a peak in the $Q(\hat{S}, t)$ function for the $21^{-}$ screw axis at $(-.003,0.003,0.0)$.

In certain cases, rotation (and screw) axes can produce pseudo-glide planes. A point at $x, y, z$, operated on by a $2_{1}-$ screw axis parallel to b located at $\left(0.25,0.0, t_{z}\right)$, will produce a point at $1 / 2-x, 1 / 2+y, 2 t_{z}-z$. Assuming a mirror perpendicular to a remains from the Patterson, a pseudo-peak at $1 / 2+x, 1 / 2+y, 2 t_{z}-z$ is generated which is "related" to the original peak at $x, y, z$ by a pseudo-n-glide perpendicular to c at $\left(0.0,0.0, t_{z}\right)$. Since the location of the $2_{1}$-screw axis parallel to $b$ in the $P$ image is located at $(0.25,0.0$, $0.39)$, the peak at $(0.0,0.0,0.388)$ in the $Q(\hat{s}, t)$ function for the $n-g l i d e$ is the result of the interaction of pseudosymmetry with the $2_{1}$-sorew axis in the $P$ image.

since there is apparently a large amount of pseudosymmetry remaining in the superposition map, it is reasonable to expert that the $G_{h}$ would not be a good approximation to the $E_{h}$. When the $G_{h}$ are transformed and the phases of the some of the largest $E_{h}$ assigned and then refined using the EG relationship, the electron density map produced is uninterpretable. However, the fact that the locations of the symmetry elements are known was used to determine the locations of the symmetry-related $P$ atoms. The vectors between the Fe atom and the four symmetryrelated $P$ atoms were chen used in a multiple weighted 
superposition. The list of peaks in the superposition map was reduced using the program TRIM. The 400 highest peaks in the list were checked for $n$-glide-related peaks in the list (using a tolerance for matching of $0.6 \mathrm{~A}$ ), then the reduced list was checked for c-glide-related peaks, and the new reduced 1 ist so obtained was checked for 21 -screwrelated peaks. The Fourier transform coefficients of this new superposition map were determined and the $Q(\hat{S}, t)$ and $S(\hat{S}, t)$ functions for the three symmetry operators, c-glide perpendicular to a, n-glide perpendicular to $c$, and $2_{1-s c r e w}$ axis parallel to $b$, were calculated. Plots of the $Q(\hat{s}, t)$ function for symmetry planes perpendicular to a and $c$ and the $2{ }_{1}$-screw axis parallel to $b$ are shown in Figure 5.10 , Figure 5.11 and Figure 5.12 , respectively.

As can be seen in these plots, the amount of pseudosymmetry in the superposition map has been signiflcantly reduced. The $G_{h}$ were transformed according to equation (3.2) and the phases of some of the largest $E_{h}$ assigned by direct transfer. The phases of those $E_{h}$ with $\left|E_{h}\right|>1.2$ were refined using the EG relation and an electron density map calculated. Figure 5.13 shows the projection of the electron density map onto the least-squares plane. A sizable part of the molecule can be determined by examination. The HYPAD atomic positions, along with the peak number and peak heights are given in Table 5.7, and the refined atomic positions are given in Table 5.8. A 
0
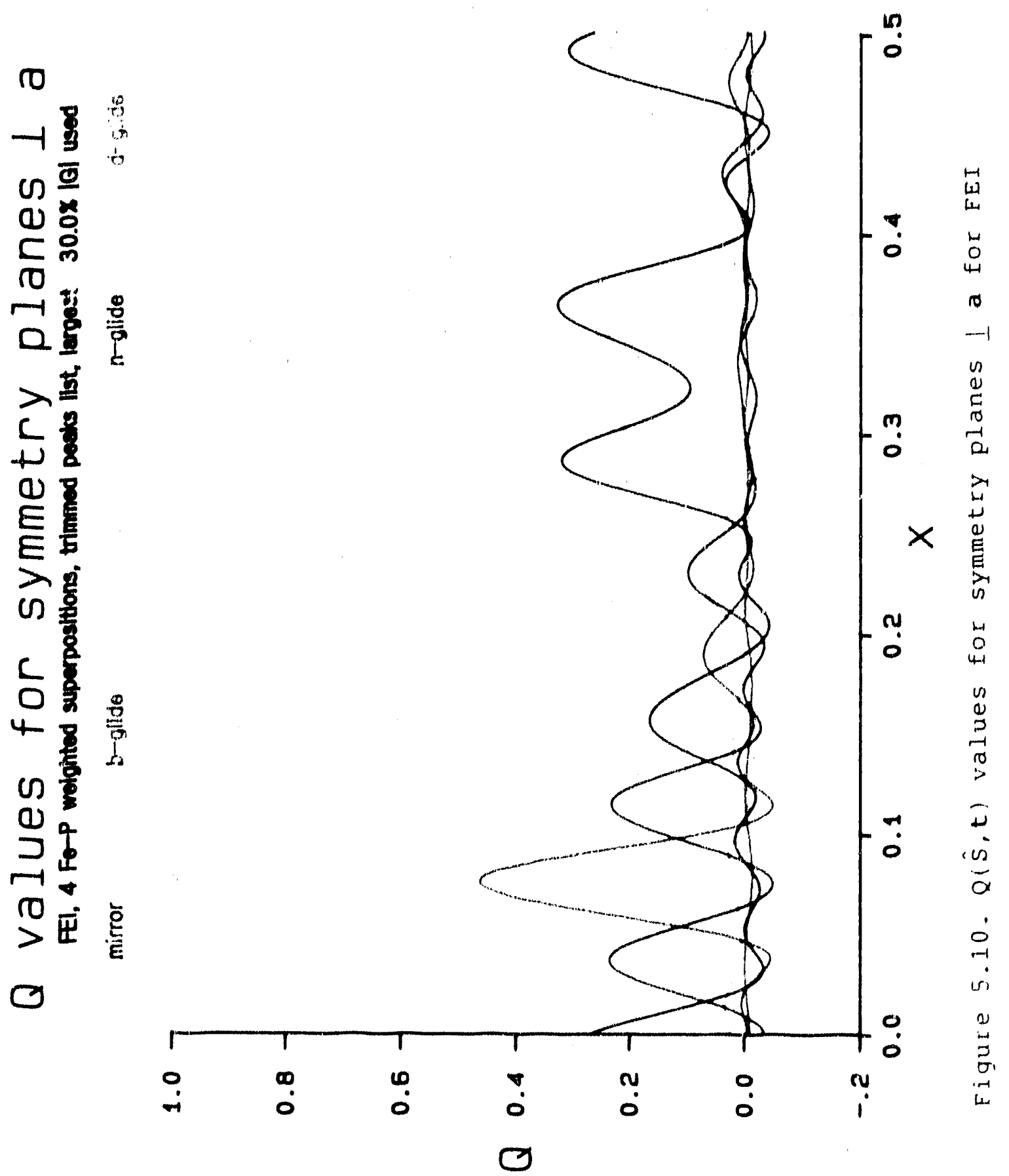


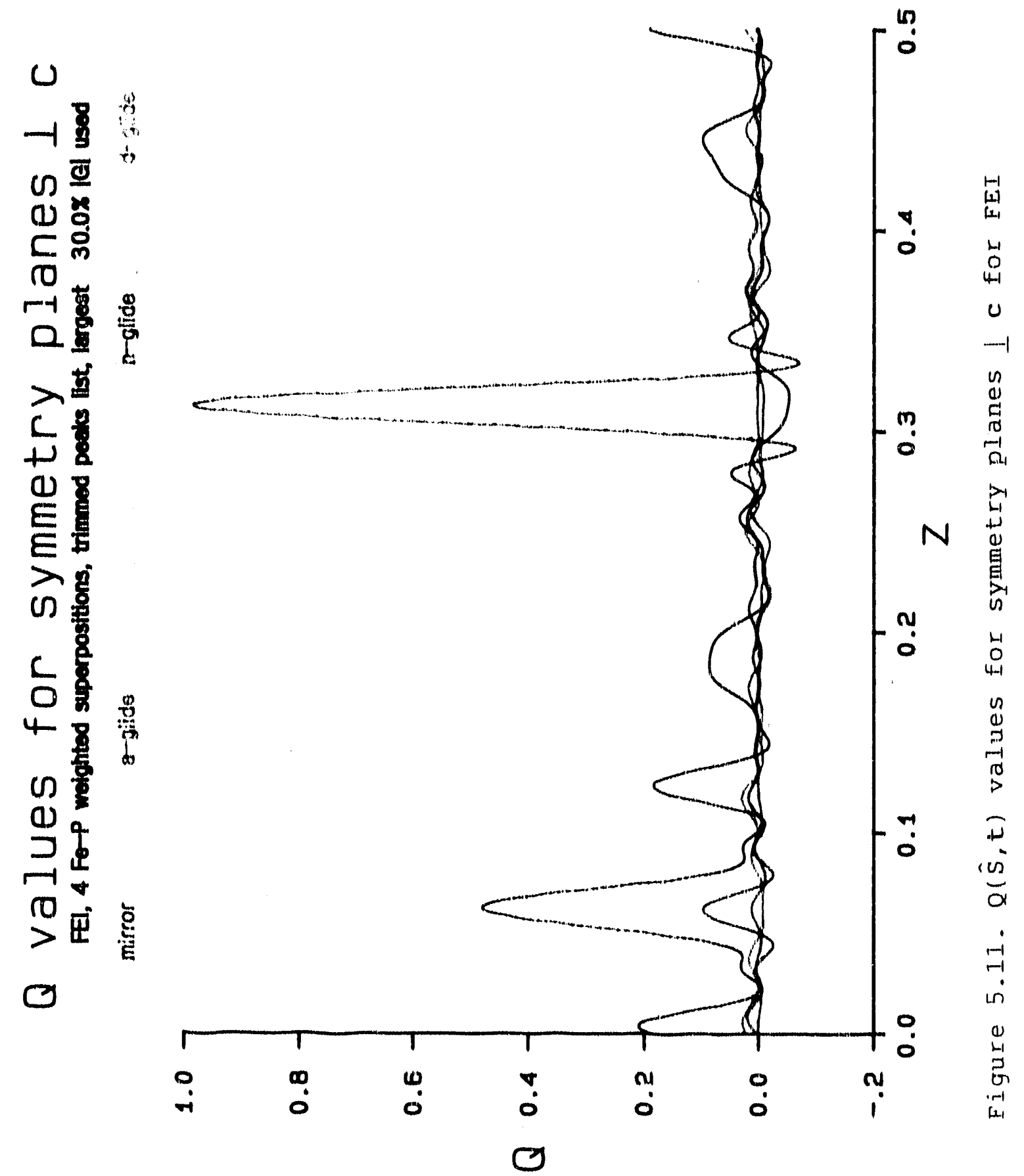




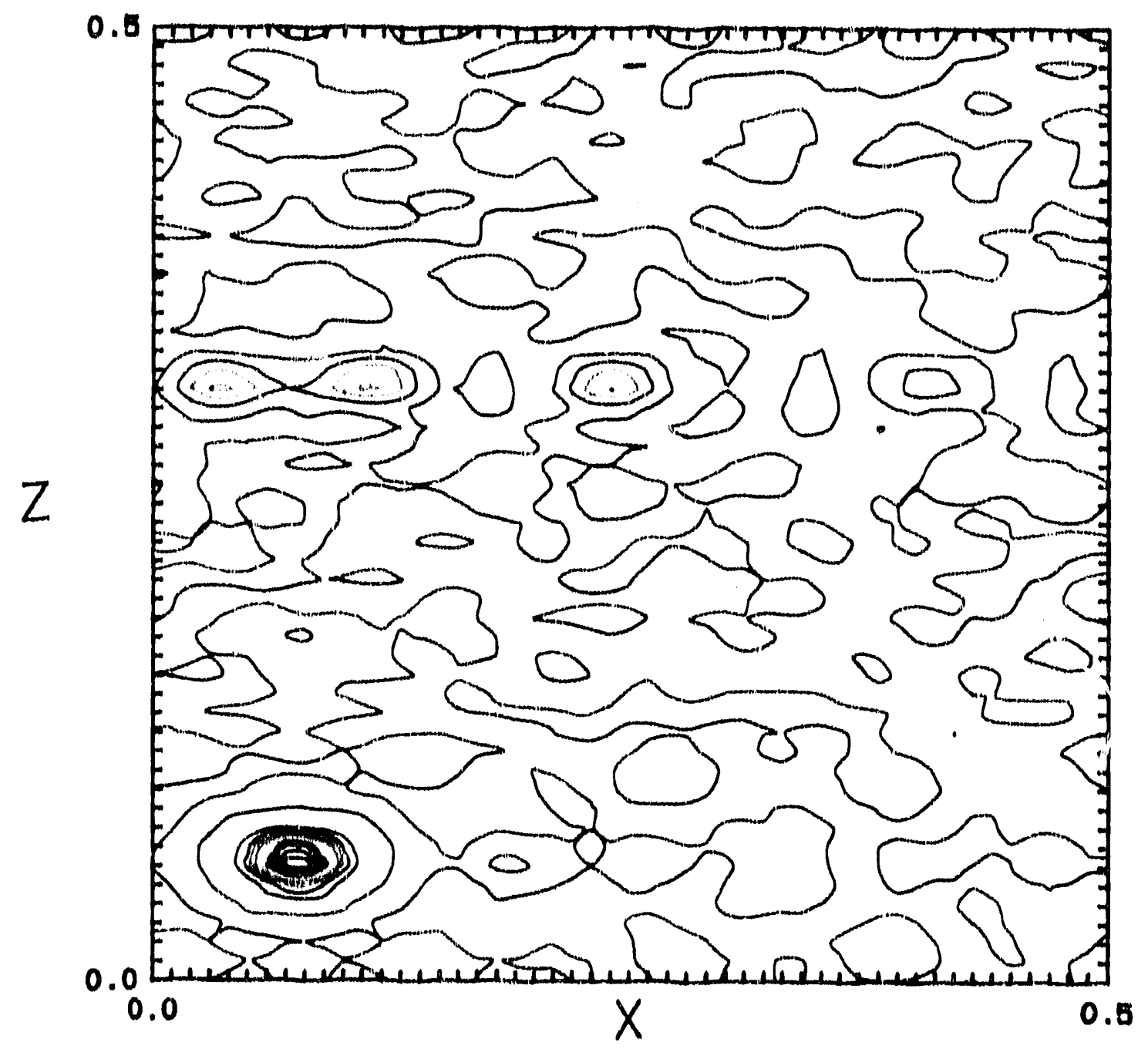

Figure 5.12. $Q(\hat{S}, t)$ values for 21 -screw axis || b for FEI 


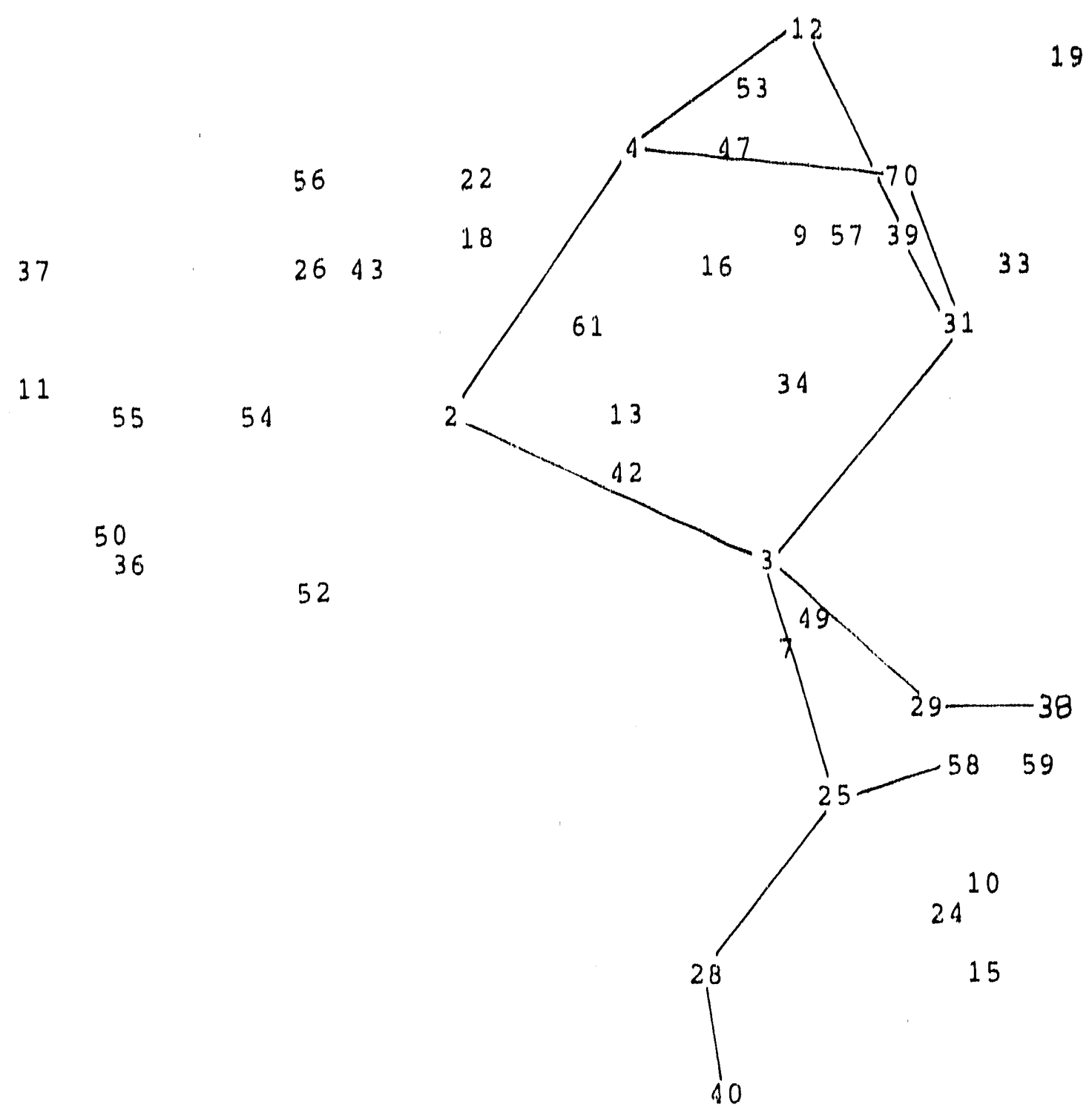

Figure 5.13. HYPAD-generated projection of $\mathrm{FeP}_{2} \mathrm{OC}_{3} 2_{31} \mathrm{H}_{3}$ 
Table 5.7. HYPAD Atomic Coordinatesa $\left(\times 10^{4}\right)$ for FeP $\mathrm{OC}_{32} \mathrm{H}_{31} \mathrm{I}$

\begin{tabular}{lrrrrr}
\hline atom & \# & pk.ht & x & y & z \\
\hline I & 1 & 359 & 2072 & 3141 & 5015 \\
$\mathrm{Fe}$ & 2 & 144 & -4223 & 5008 & 4399 \\
$\mathrm{P} 1$ & 3 & 87 & -2892 & 3941 & 4695 \\
$\mathrm{P} 2$ & 4 & 77 & -2460 & 5425 & 3858 \\
$\mathrm{C} 23$ & 12 & 36 & -914 & 5764 & 4388 \\
$\mathrm{C} 13$ & 25 & 23 & -2838 & 3459 & 5748 \\
$\mathrm{C} 14$ & 28 & 23 & -4028 & 3159 & 5997 \\
C7 & 29 & 23 & -2867 & 2847 & 428 \\
$\mathrm{C} 19$ & 31 & 22 & -1029 & 4279 & 4411 \\
$\mathrm{C} 8$ & 38 & 20 & 5846 & 2619 & 3911 \\
$\mathrm{C} 24$ & 39 & 20 & -785 & 5134 & 4995 \\
$\mathrm{C} 15$ & 40 & 20 & -4078 & 2978 & 6688 \\
$\mathrm{C} 18$ & 58 & 16 & -1825 & 3587 & 6194 \\
$\mathrm{C} 20$ & 70 & 15 & -1155 & 4552 & 3634 \\
& & & & & \\
\hline
\end{tabular}

atomic coordinates are given as fractions of the unit cell. 
Table 5.8. Refined Atomic Coordinatesa $\left(\times 10^{4}\right)$ for $\mathrm{FeP} 2 \mathrm{OC}_{32} \mathrm{H}_{31} \mathrm{I}$

\begin{tabular}{crrr}
\hline atom & \multicolumn{1}{c}{ x } & \multicolumn{1}{c}{ z } \\
\hline I & $2059(1)^{b}$ & $3175(2)$ & $5058(1)$ \\
Fe & $-4235(3)$ & 5000 & $4429(2)$ \\
P1 & $-2878(6)$ & $3965(4)$ & $4714(3)$ \\
P2 & $-2467(6)$ & $5440(4)$ & $3853(3)$ \\
C23 & $-1022(21)$ & $5838(12)$ & $4369(12)$ \\
C13 & $-2829(22)$ & $3608(12)$ & $5658(12)$ \\
C14 & $-3993(21)$ & $3306(16)$ & $5917(12)$ \\
C7 & $-3007(22)$ & $3011(13)$ & $4231(10)$ \\
C19 & $-1177(20)$ & $4312(12)$ & $4506(11)$ \\
C8 & $-4187(23)$ & $2774(14)$ & $3920(12)$ \\
C2A & $-855(18)$ & $513(12)$ & $4903(11)$ \\
C15 & $-3975(25)$ & $2998(15)$ & $6695(13)$ \\
C18 & $-1643(23)$ & $3717(15)$ & $6113(14)$ \\
C20 & $-1272(20)$ & $4630(12)$ & $3651(11)$
\end{tabular}

Aatomic coordinates are given as fractions of the unit cell.

bestimated standard deviations for the refined coordinates are given in parentheses for the least significant digit. 
Tablle 5.9. comprative bond distances for FeP $\mathrm{OC}_{32} \mathrm{H}_{31}$

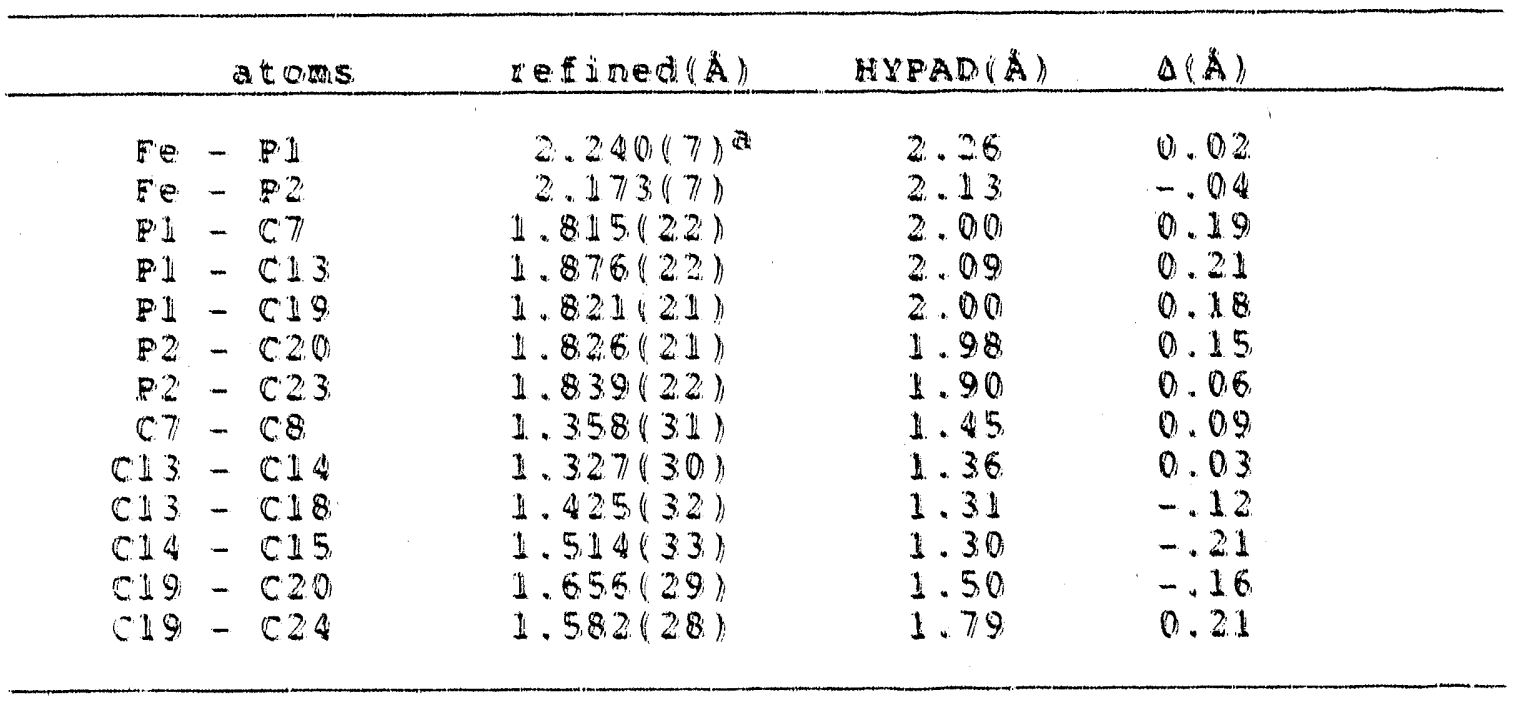

astimated standard deviations for the refined distances are given in parentheses for the least significant digit. 
comparative table of bond distances can be found in Table 5.9.

A least-squares refinement of the atom positions, with fixed isotropic thermal parameters, obtained from HYPAD resulted in a cesidual index (f factor) of 16.8 . This represents an excellent start! The remainder of the molecule, except hydrogen atoms, was found in the first electron density map calculated from these results.

These results show a that good initial model for the struccure of a fairly complex compound can be derived from the superposition map. The bond distances in the HYPADgernegated fragment are within $0.21 \mathrm{~A}$ of the refined bond distances. The ercors in the distances of bonds containing c atoms are less than ten times the standard deviation of the refined distances. Considering the fact that the nomina: resolution in the map was on the order of 0.26 D. IlA and that the only small portion of the $E_{h}$ were used in generating the electron density map, this result is quite satisfactory. These gesults also illustrate one of the major difficulties of superposition techniques. that of pseudo-symatry. However, even with the presence of a large amount of pseudo-symmetry. HyPAD was able to determine the corgect symety and the locations of the symmety elements. Although, in this case, the single-superposicion map does not give a good phase set tor the $E_{h}$, a method for obtaining a good phase set does present itself. Use of the locations 
of the symmetry operators to find more vectors to use for additional superpositions is a trivial procedure, and, as this case shows, it can have tremendous rewards.

$$
\text { HYPAD Solution of } \mathrm{Ni}\left(\mathrm{C}_{6} \mathrm{O}_{3} \mathrm{H}_{9}\right)_{2} \mathrm{Cl}_{2}
$$

This compound (NIT) - shown in Figure 5.14 - is a slightly more complex organo-metallic compound than $\mathrm{Cr}(\mathrm{CO})_{3} \mathrm{C}_{6} \mathrm{H}_{6}$. It was originally solved using the "heavyatom" method by Bill Jenson's group (Department of Chemistry, south Dakota state University). The HYPAD solution of this compound turned out to be very routine.

This compound crystallizes on the monoclinic crystal system. The extinction conditions on the intensities uniquely indicated that the space group was the centrosymmetric choice $\mathrm{Pa}_{1} \mathrm{C}$. A vector corresponding to a $\mathrm{Ni}-\mathrm{O}$ vector was chosen as the shift vector and an unweighted superposition was done. The Fourier transform coefficients of the superposition map were determined and the $Q(\hat{s}, t)$ and $s(\hat{s}, t)$ functions were calculated for the $2,-s c r e w$ axis parallel to $b$, the $c-g l i d e$ plane perpendicular to $b$ and the inversion operator. plots of the $Q(\hat{S}, t)$ function for the 21 -screw axis and symmetry planes perpendicular to b are shown in Figures 5.16 and 5.15 , respectively. Table 5.10 lists the translations for the operators, as well as the 
18

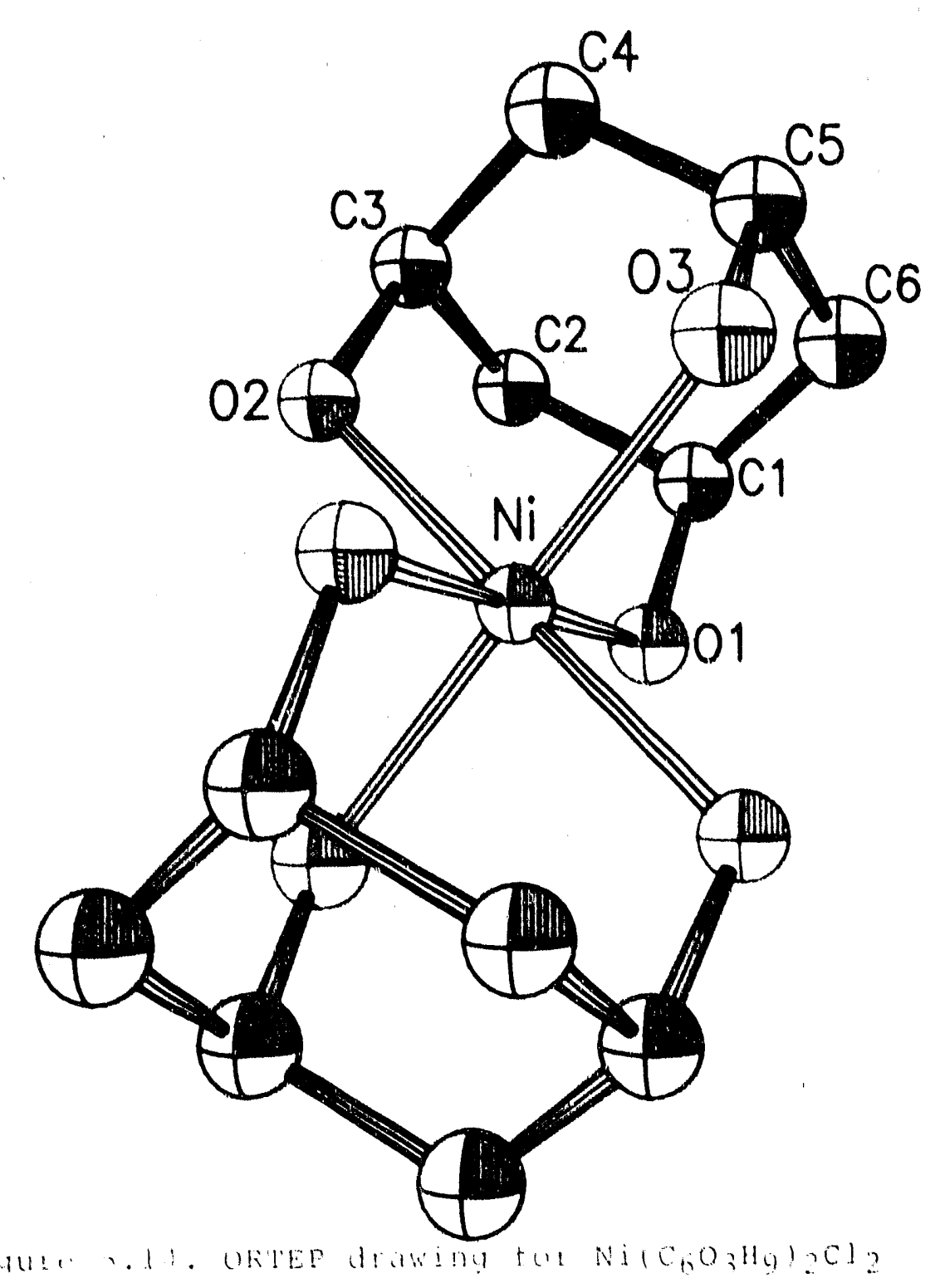

$\mathrm{Cl}$ 


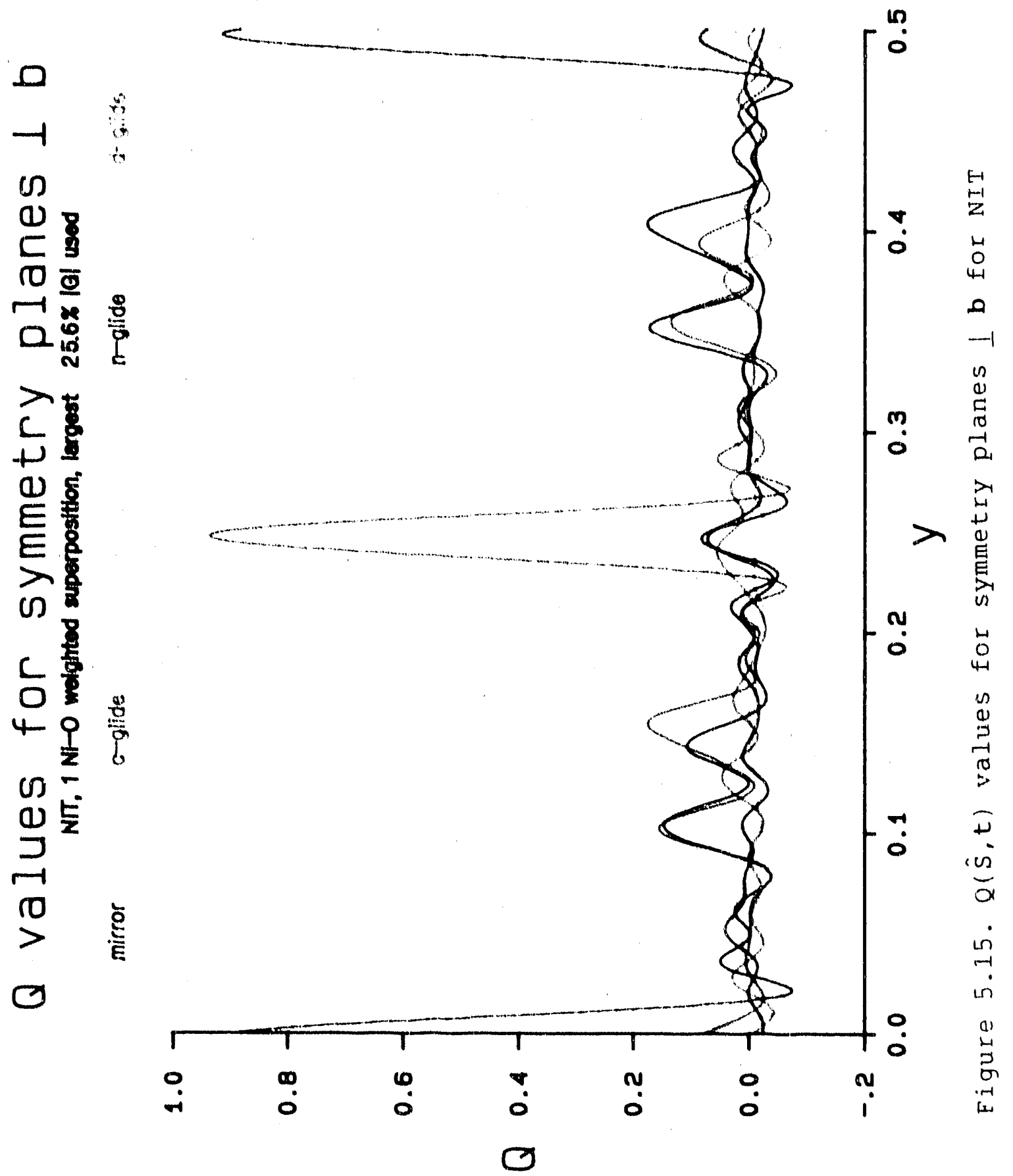




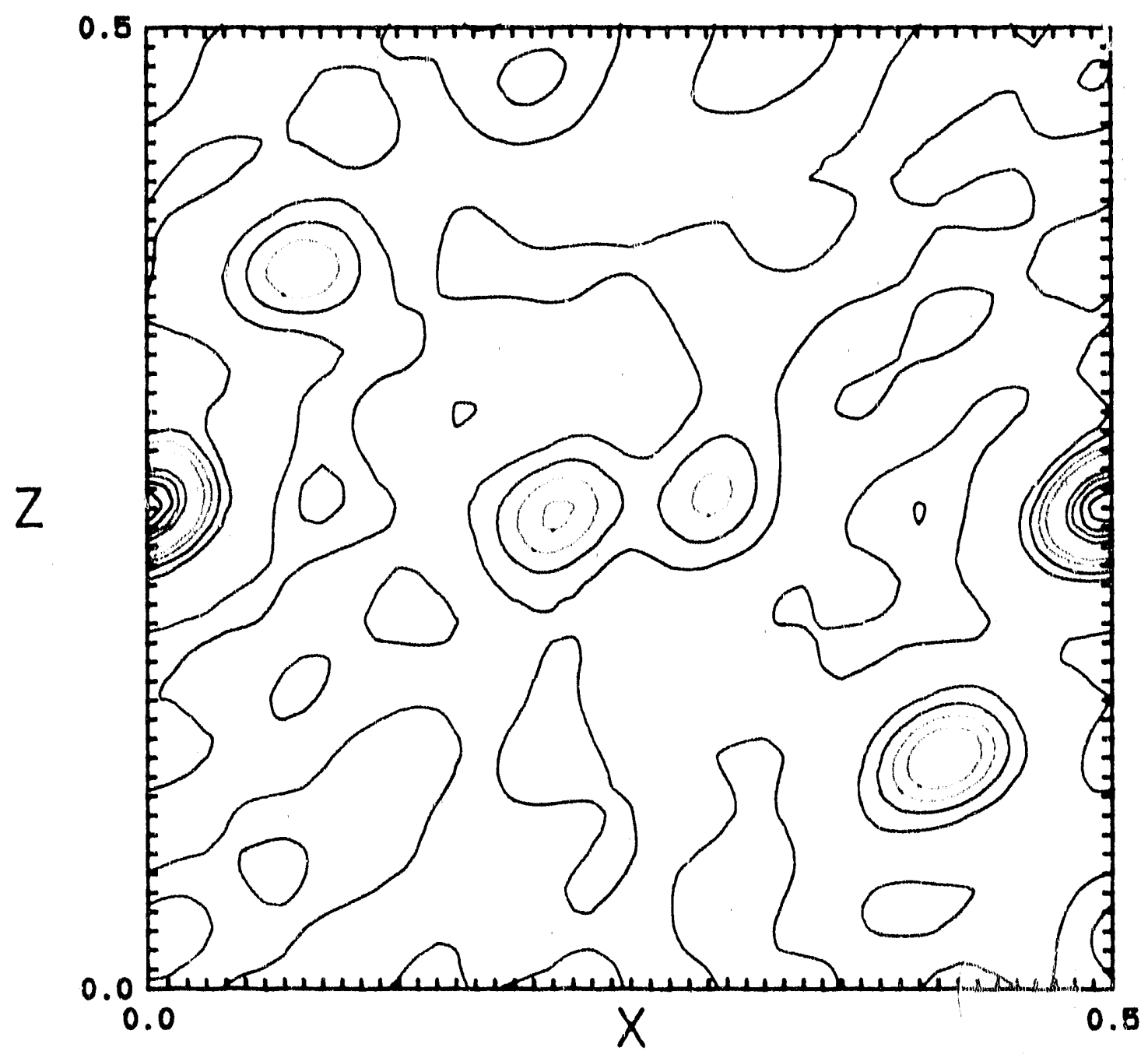

Figure 5.16. $Q(\hat{S}, t)$ values for $21 \cdots s c r e w$ axis $\|$ b for NIT 
Table 5.10. Symmetry Operator Locations for $\mathrm{Ni}\left(\mathrm{C}_{6} \mathrm{O}_{3} \mathrm{H}_{9}\right)_{2} \mathrm{Cl}_{2}$

\begin{tabular}{ccccc}
\hline symmetry operator & $t^{2}$ & $Q(\hat{s}, t)$ & $s(\hat{s}, t)$ \\
\hline$-x, 1 / 2+y,-z$ & $(-.0024,0.0,0.2493)$ & 0.8845 & 0.0140 \\
$x,-y, z+1 / 2$ & $(0.0,0.2475,0.0)$ & 0.9343 & 0.0073 \\
$-x,-y,-z$ & $(-.0024,-.0029,-.0003)$ & 0.8317 & 0.0213 \\
\hline
\end{tabular}

aranslations are given in fractions of a unit cell.

values of the $Q(\hat{S}, t)$ and $s(\hat{s}, t)$ functions for the translations.

There is essentially one peak in the $Q(\hat{S}, t)$ function for the c-glide plane, and it corresponds to the location of the glide plane in the $\mathrm{Ni}$ image. Similarly, there is one large peak in the $Q(\hat{S}, t)$ function for the 21 -screw axis. For this compound, the $\mathrm{Ni}$ atom lies on an inversion center. Thus, all vectors between the $\mathrm{NI}$ atom and all other atoms in the cell (except the other $\mathrm{Ni}$ atom) are multiple vectors.

According to superposition theory, there should be $2 \mathrm{~m}$ images in the superposition map, where $m$ is the multiplicity of the superposition vector. The fact that a weighted superposition was done only suppresses the o images relative to the $\mathrm{Ni}$ images, and does not eliminate these images. The vector used was of multiplicity two (the vector from the $\mathrm{Ni}$ atom to the $O$ atom is the same as the vector from the inversion-related $O$ atom to the $\mathrm{Ni}$ ), so there should be four images in the superposition map. However, two of the 
images, those with Ni at the tail of the shift vectors, exactly superimpose on top of one another, which leaves a result of three images.

The peaks in the $Q(\hat{s}, t)$ function for the $c-g l i d e$ plane at 0.16 and 0.35 are the locations of the glide plare in the o images. The large peak at the origin of the $Q(\hat{S}, t)$ function for the mirror plane indicates that there is still a sizable amount of pseudo-symmetry left in the superposition map. The $Q(\hat{s}, t)$ function for the $2_{1}$-screw axis has smaller peaks at $(0.08,0.0,0.38)$ and $(0.42,0.0$, 0.12 ) which are the locations of the $21^{-s c r e w}$ axis in the 0 images.

Since the $\mathrm{Ni}$ atom lies on an inversion center, and by convention, the origin of the electron density map in a centrosymmetric space group is taken to be on an inversion center, there is no need to transform the $G_{h}$ according to equation (3.2). The $G_{h}$ that were symmetry-related were averaged. The phases of 196 of the largest $E_{h}$ were set by direct transfer of the phases of the corresponding $G_{h}$. The $E_{h}$ were refined using the EG relationship and an electron density map was calculated. Figure 5.17 shows the projection of the electron density map onto the leastsquares plane. The molecule is clearly identifiable in this figure. The $\mathrm{Cl}$ atoms are present in the electron density map, but are not included in the projection because they are not bound to any part of the structure. All of the unique 


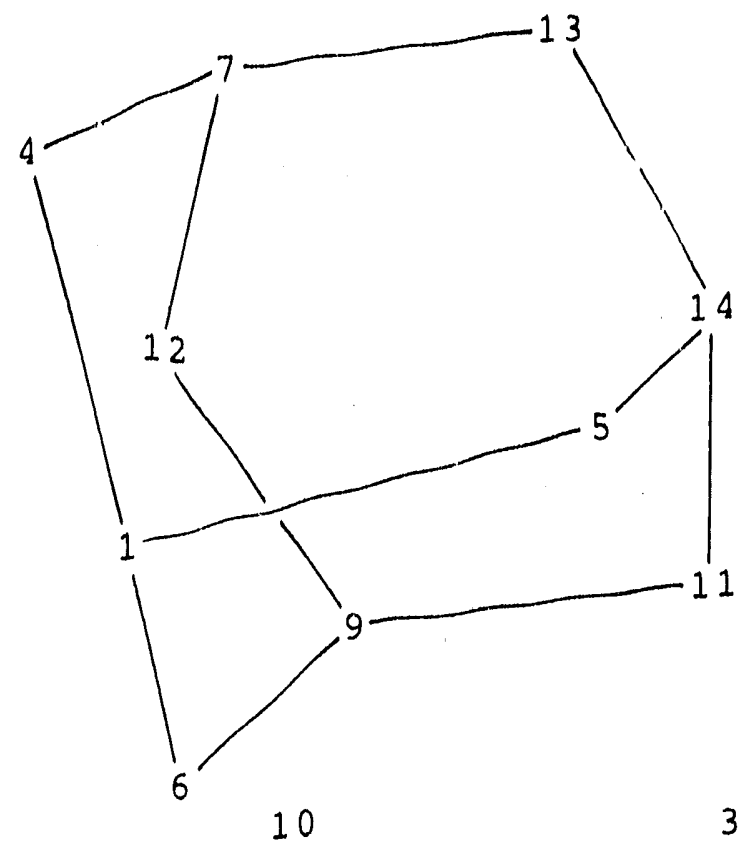

8

Figure 5.17. HYPAD projection of $\mathrm{Ni}\left(\mathrm{C}_{6} \mathrm{O}_{3} \mathrm{Hg}\right)_{2} \mathrm{Cl}_{2}$ 
Table 5.11. HYPAD Atomic Coordinatesa $\left(\times 10^{4}\right)$ for $\mathrm{Ni}\left(\mathrm{C}_{6} \mathrm{O}_{3} \mathrm{H}_{9}\right)_{2} \mathrm{Cl}_{2}$

\begin{tabular}{rrrrrr}
\hline atom & \# & pk.ht & \multicolumn{1}{c}{ x } & \multicolumn{1}{c}{ y } \\
\hline Ni & 1 & 529 & -9 & 30 & 10 \\
C1 & 2 & 217 & -4931 & -1441 & -2259 \\
01 & 5 & 92 & 1576 & 147 & 2535 \\
02 & 4 & 94 & -1933 & -817 & 940 \\
03 & 6 & 90 & 1902 & -962 & -260 \\
C1 & 14 & 47 & 2184 & -525 & 3543 \\
C2 & 13 & 47 & 265 & -956 & 3735 \\
C3 & 7 & 68 & -935 & -1418 & 2210 \\
C4 & 12 & 50 & 491 & -2002 & 1351 \\
C5 & 9 & 56 & 2469 & -1582 & 1180 \\
C6 & 11 & 52 & 3736 & -1112 & 2850 \\
\end{tabular}
cell.

atomic coordinates are given as fractions of the unit

Tabje 5.12. Refined Atomic Coordinatesa $\left(\times 10^{4}\right)$ for $\mathrm{Ni}\left(\mathrm{C}_{6} \mathrm{O}_{3} \mathrm{H}_{9}\right){ }_{2} \mathrm{Cl}_{2}$

\begin{tabular}{|c|c|c|c|}
\hline atom & $x$ & $y$ & $z$ \\
\hline $\begin{array}{l}\mathrm{Ni} \\
\mathrm{CH} \\
\mathrm{O} 1 \\
\mathrm{O} 2 \\
\mathrm{O} 3 \\
\mathrm{C} 1 \\
\mathrm{C} 2 \\
\mathrm{C} 3 \\
\mathrm{C} 4 \\
\mathrm{C} 5 \\
\mathrm{C} 6\end{array}$ & $\begin{array}{c}0 \\
-4927(2)^{b} \\
1591(4) \\
-1877(4) \\
1877(5) \\
2270(6) \\
307(6) \\
-888(6) \\
533(7) \\
2495(7) \\
3775(7)\end{array}$ & $\begin{array}{r}0 \\
-1462(7) \\
173(2) \\
-818(2) \\
-987(2) \\
-553(3) \\
-998(3) \\
-1447(3) \\
-2009(3) \\
-1576(3) \\
-1120(3)\end{array}$ & $\begin{array}{r}0 \\
-2265(1) \\
2495(3) \\
945(3) \\
-202(3) \\
3579(5) \\
3802(5) \\
2194(5) \\
1460(5) \\
1211(5) \\
2819(5)\end{array}$ \\
\hline
\end{tabular}
cell.

a tomic coordinates are given as fractions of the unit

bestimated standard deviations for the refined coordinates are given in parentheses for the least significant digit. 
Table 5.13. Comparative bond distances for $\mathrm{N} 1\left(\mathrm{C}_{6} \mathrm{O}_{3} \mathrm{H}_{9}\right)_{2} \mathrm{Cl}_{2}$

\begin{tabular}{cccc}
\hline atoms & refined $(A)$ & HYPAD $(A)$ & $\Delta(A)$ \\
\hline$N i-01$ & $2.048(4) a$ & 2.05 & 0.00 \\
$N 1-02$ & $2.056(4)$ & 2.06 & 0.00 \\
$N 1-03$ & $2.029(5)$ & 2.06 & 0.03 \\
$01-C 1$ & $1.457(8)$ & 1.35 & -.11 \\
$02-C 3$ & $1.466(8)$ & 1.44 & -.03 \\
$03-C 5$ & $1.470(8)$ & 1.50 & 0.03 \\
$C 1-C 2$ & $1.537(10)$ & 1.47 & -.07 \\
$C 1-C 6$ & $1.532(10)$ & 1.58 & 0.05 \\
$C 2-C 3$ & $1.533(9)$ & 1.48 & -.05 \\
$C 3-C 4$ & $1.518(10)$ & 1.59 & 0.07 \\
$C 4-C 5$ & $1.526(11)$ & 1.49 & -.04 \\
$C 5-C 6$ & $1.518(10)$ & 1.59 & 0.07 \\
\hline
\end{tabular}

aEstimated standard deviations for the refined distances are given in parentheses for the least significant digit. 
Table 5.14. Comparative bond angles for $\mathrm{Ni}\left(\mathrm{C}_{6} \mathrm{O}_{3} \mathrm{H}_{9}\right)_{2} \mathrm{Cl}_{2}$

\begin{tabular}{|c|c|c|c|c|c|}
\hline \multicolumn{3}{|c|}{ atoms } & refined $\left({ }^{\circ}\right)$ & HYPAD $\left({ }^{\circ}\right)$ & $\Delta\left(0^{0}\right)$ \\
\hline $\begin{array}{l}01 \\
01 \\
\mathrm{O} 2 \\
\mathrm{~N} 1 \\
\mathrm{~N} 1 \\
\mathrm{NI} \\
\mathrm{O} 1 \\
01 \\
\mathrm{C} 2 \\
\mathrm{C} 1 \\
02 \\
\mathrm{O} 2 \\
\mathrm{C} 2 \\
\mathrm{C} 3 \\
\mathrm{O} \\
\mathrm{O} 3 \\
\mathrm{C} 4 \\
\mathrm{C} 5\end{array}$ & $\begin{array}{l}-N 1 \\
-N 1 \\
-N 1 \\
-O 1 \\
-O 2 \\
-O 3 \\
-C 1 \\
-C 1 \\
-C 1 \\
-C 2 \\
-C 3 \\
-C 3 \\
-C 3 \\
-C 4 \\
-C 5 \\
-C 5 \\
-C 5 \\
-C 6\end{array}$ & 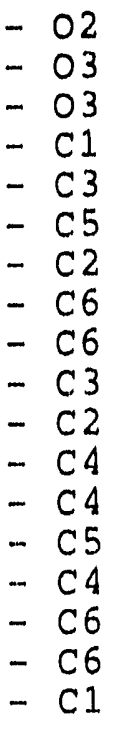 & $\begin{array}{r}80.55(17)^{a} \\
90.64(18) \\
87.79(18) \\
119.37(35) \\
119.71(38) \\
120.59(38) \\
107.04(15) \\
111.28(51) \\
111.58(56) \\
113.72(53) \\
107.07(49) \\
110.33(50) \\
112.32(58) \\
114.24(56) \\
107.03(56) \\
109.88(54) \\
112.16(56) \\
114.38(59)\end{array}$ & $\begin{array}{l}84.9 \\
89.9 \\
88.3 \\
121.8 \\
118.3 \\
118.1 \\
107.5 \\
112.6 \\
113.2 \\
116.3 \\
108.2 \\
107.4 \\
113.7 \\
112.8 \\
108.0 \\
108.7 \\
115.1 \\
109.3\end{array}$ & $\begin{array}{r}-1.7 \\
0.7 \\
0.5 \\
2.4 \\
-1.4 \\
-2.5 \\
0.5 \\
1.3 \\
1.6 \\
2.6 \\
1.1 \\
-2.9 \\
1.4 \\
-1.4 \\
1.4 \\
-1.2 \\
2.9 \\
-5.1\end{array}$ \\
\hline
\end{tabular}

astimated standard deviations for the refined angles are given in parentheses for the least significant digits. 
non-hydrogen atoms in the molecule are found in the top is peaks. The HYPAD atomic positions, peak number and peak helghts are given in Table 5.11 and the refined atomic positions are given in Table 5.12. Tables 5.13 and 5.14 compare the bond distances and bond angles obtained from the HYPAD and refined positions. Again, the HYPAD-generated bond distances are within 0.11 A of the refined distances and the HYPAD-generated bond angles are generally within $3^{\circ}$ of the refined angles. Considering the fact that the nominal resolution in the map was $0.25-0.41 \mathrm{~A}$, these results are excelient.

The symmetry averaging of the $G_{h}$ is important as it removes many peaks in the electron density map which are "related" to actual peaks by pseudo-symmetry. Without symmetry averaging, the unique atoms are found in the top 19 peaks in the map. However, many of the extraneous peaks are pseudo-related to actual peaks by a mirror or a 2-fold axts, neither of which is present in the space group $\mathrm{P} 21 / \mathrm{C}$. Many of these pseudo-peaks are more prominent than the actual peaks. When the $G_{h}$ are averaged, only one pseudo-related peak remains, a pseudo-chlorine, at approximately the peak helght of the actual chlorine atom. 
HYPAD SOlution of $\left(\mathrm{NHC}_{5} \mathrm{H}_{5}\right)_{2} \mathrm{I}_{10}$

This compound formed as a side product of the reaction that produced crystals of $\left(\mathrm{NHC}_{5} \mathrm{H}_{5}\right) \mathrm{SbI}_{4}$ (see Appendix B). one of more interesting physical characteristics of this compound is that it forms extremely long needle-shaped crystals, several centimeters in length. It was inftially thought that these crystals were an alternate crystalline form of the antimony compound. However, the structure, shown in Figure 5.18 , indicates otherwise.

This compound crystallizes in the orthorhomblc crystal system. The extinction conditions upon the observed intensities only indicated the presence of a b-glide plane perpendicular to c. Thus, there were three possible space groups: Pmmb (centrosymmetric), P2mb (non-centrosymmetric) and $\operatorname{Pm} 2_{1} b$ (non-centrosymmetric).

Direct methods failed to provide a solution in any of the three possible space groups. Attempts to analyze the patterson map by hand were frultless. A vector which seemed a likely candidate for an $\mathrm{Sb}-\mathrm{I}$ vector was used for an unweighted superposition, since the weighting factor for this vector is almost unity. The fourier transform coefficients of the superposition map were calculated. The locations of all of the possible symmetry elements in the three space groups are listed in Table 5.15, along with $Q(\hat{S}, t)$ and "mirrored" $S(\hat{S}, t)$ values. Translations 


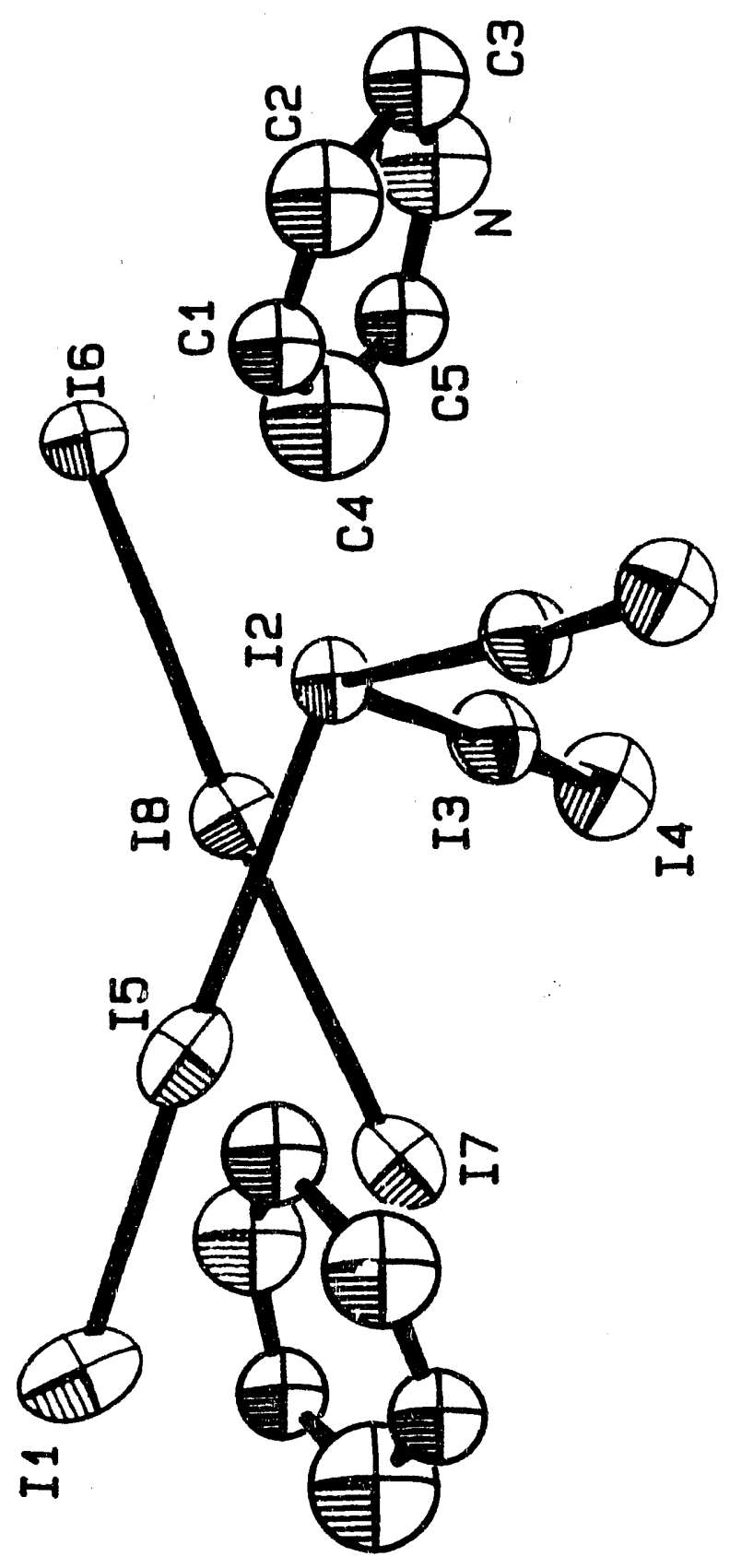

年 


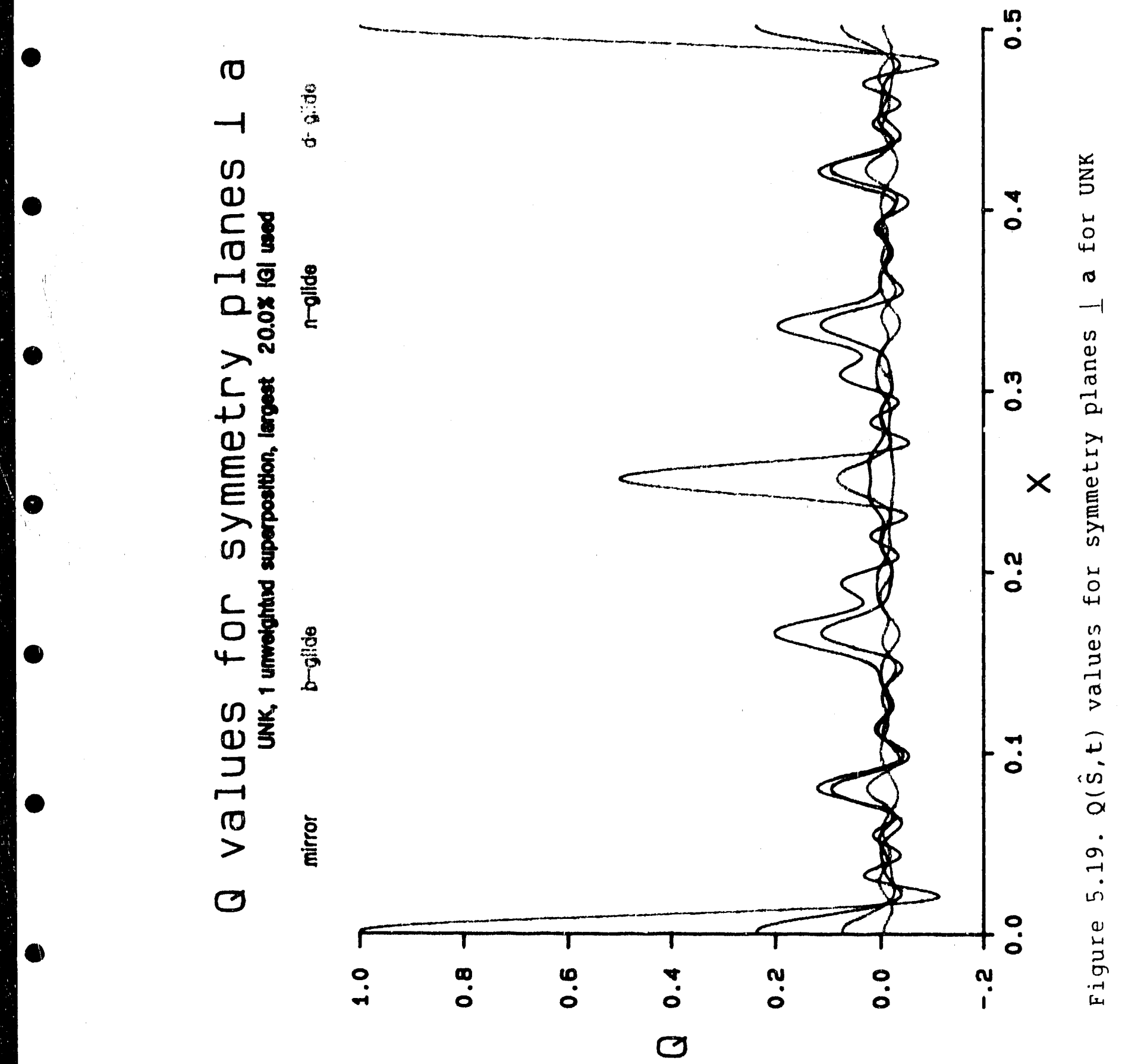


Figure 5.20. $Q(\hat{s}, t)$ values for $21_{1}$-screw axis $\|$ b for UNK 
0

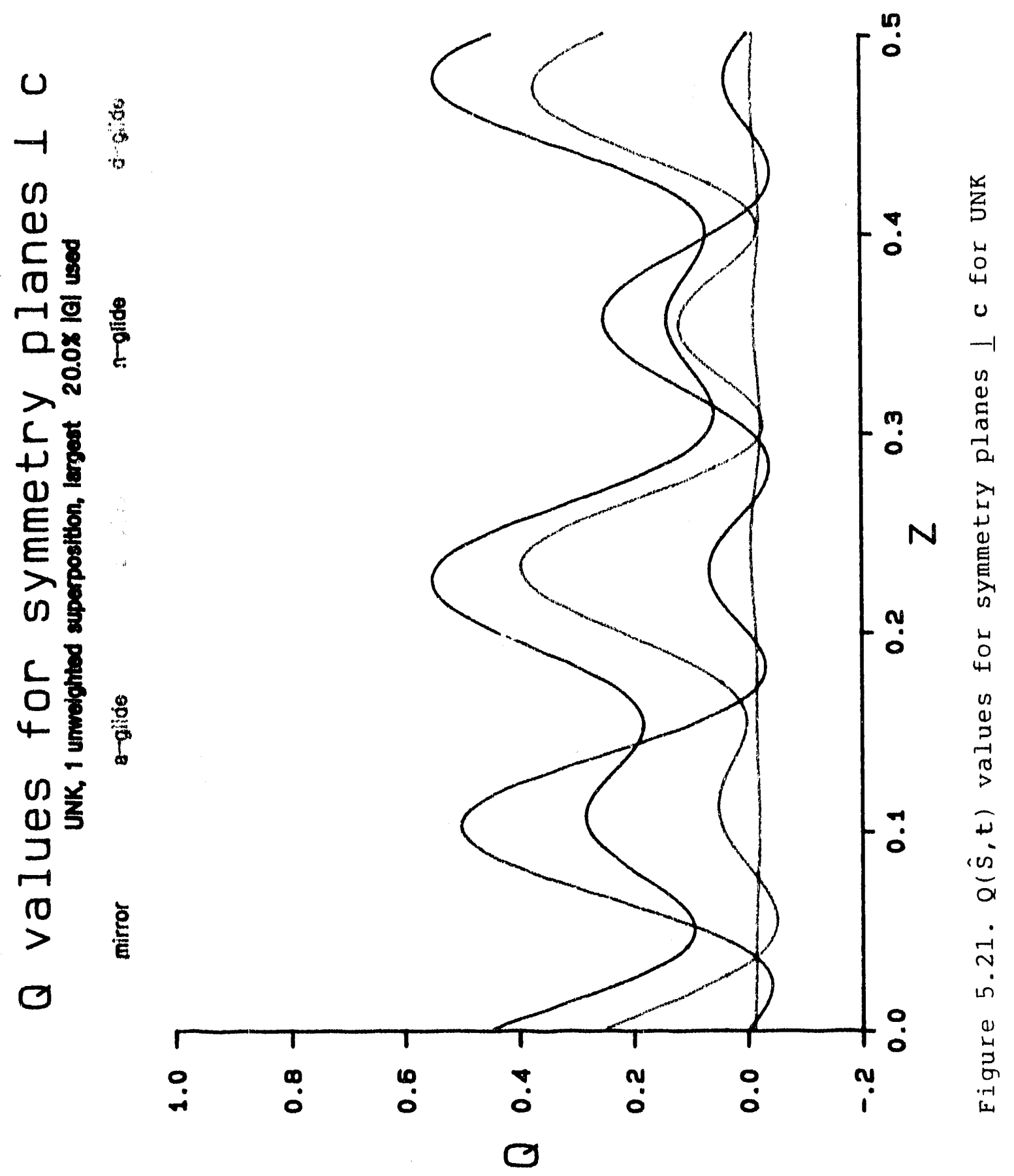


Table 5.15. Symmetry Operator Locations for $\left(\mathrm{NHC}_{5} \mathrm{H}_{5}\right)_{2} \mathrm{I}_{10}$

\begin{tabular}{|c|c|c|c|}
\hline symmetry operator & $t^{a}$ & $Q(\hat{S}, t)$ & $s(\hat{s}, t)$ \\
\hline $\begin{array}{l}x,-y,-z \\
-x, y+1 / 2, z \\
-x,-y, z \\
-x, y, z \\
x,-y, z \\
x, y+1 / 2,-z \\
-x,-y,-z\end{array}$ & $\begin{array}{c}(0.0,0.4220,0.3517) \\
(0.0,0.0,0.1011) \\
(0.0,0.1728,0.0) \\
(0.2500,0.0,0.0) \\
(0.0,0.1728,0.0) \\
(0.0,0.0,0.1011) \\
(0.0000,0.4220,0.3517)\end{array}$ & $\begin{array}{l}0.9772 \\
0.5232 \\
0.5459 \\
0.5018 \\
0.5465 \\
0.5236 \\
0.9766\end{array}$ & $\begin{array}{l}0.0030 \\
0.0037 \\
0.0047 \\
0.0001 \\
0.0046 \\
0.0036 \\
0.0031\end{array}$ \\
\hline
\end{tabular}

a Translations are given in fractions of a unit cell.

consistent with the b-glide plane perpendicular to c were found only for the mirror perpendicular to a and the $2_{1}$ screw axis parallel to $b$, indicating that the correct space group was $P m 21 b$. The $Q(\hat{S}, t)$ functions are plotted for the mirror perpendicular to $\mathbf{a}$, the $2_{1}$-screw axis parallel to $\mathbf{b}$ and the b-glide plane perpendicular to c in Figures 5.19, $5.20,5.21$, respectively. For this space group, the origin is located on the 21 -screw axis. The positions of the ends of the shift vector relative to the location of the 21-screw axis were calculated and used as input to a least-squares refinement. The rest of the molecule was found by successive structure factor and electron density map calculations.

Although the structure was not immediately evident from the superposition map, it is important to note that the space group and the location of the symmetry elements could 
be obtained. Even in a worst-case scenario, with an unweighted superposition and a very vague idea as to what types of atoms are in the structure, one can at least get a "foot in the door" and continue with a least-squares refinement.

HYPAD solution of $\mathrm{Cu}\left(\mathrm{C}_{6} \mathrm{O}_{3} \mathrm{H}_{9}\right) \mathrm{Cl}_{2}$

This compound (CUT), shown in Figure 5.22, was expected to be the $\mathrm{Cu}$ analogue to $\mathrm{Ni}\left(\mathrm{C}_{6} \mathrm{H}_{9} \mathrm{O}_{3}\right)_{2} \mathrm{Cl}_{2}$; however its structure turned out to be different. It was originally solved by Bill Jenson's group (Department of Chemistry, South Dakota state University) using direct methods. Although the position of the $\mathrm{Cu}$ atom can be obtained from the patterson map, it does not provide enough phase information to solve the structure via the "heavy-atom" method.

This compound crystallizes in the monoclinic crystal system. The extinction conditions on the intensities indicated the presence of a c-glide plane perpendicular to $b$ and c-centering. There are two possible space group choices: Cc (non-centrosymmetric) and $C 2 / C$ (centrosymmetric).

A vector corresponding to a $\mathrm{Cu}-0$ vector was chosen to use as a superposition shift vector. Selection of an appropriate vector was complicated by the fact that the 

$\mathrm{Cu}-\mathrm{Cl}$ bond distances are comparable to the $\mathrm{Cu}-\mathrm{O}$ bond distances. A weighted superposition was done and the Fourier transform coefficients of the map were calculated. $Q(\hat{S}, t)$ and $s(\hat{s}, t)$ functions for all of the possible symmetry eiements in both space groups were calculated and the best translation choices are listed in Table 5.16. A plot of the $Q(\hat{S}, t)$ values for symmetry planes perpendicular to $b$ is shown in Figure 5.23 .

There is still a large amount of pseudo-symmetry remaining as indicated by the large peaks in the $Q(\hat{s}, t)$ functions of the mirror and a-glide flanes, as well as the large $Q$ values for the rotation-type axes and the inversion center translations. The locations of the 2-fold axis, the 21 -screw axis laenerated from tire 2-fold axis by the Ccentering operation), and the inversion centers are not consistent with the location of the c-glide plane, indicating that the space group is Cc. The other symmetry elements are pseudo-symmetry from the patterson map which has symmetry $\mathrm{c} 2 / \mathrm{m}$.

There are two large peaks in the $c-g l i d e$ plane $Q(\hat{S}, t)$ function. The peak at 0.18 is the location of the glide plane in the cu image. The other large peak, at 0.32 is the result of pseudo-symmetry. The peak at 0.07 is the location of the glide plane in the 0 image and the peak at 0.43 is the result of pseudo-symmetry. The c-centering operation 


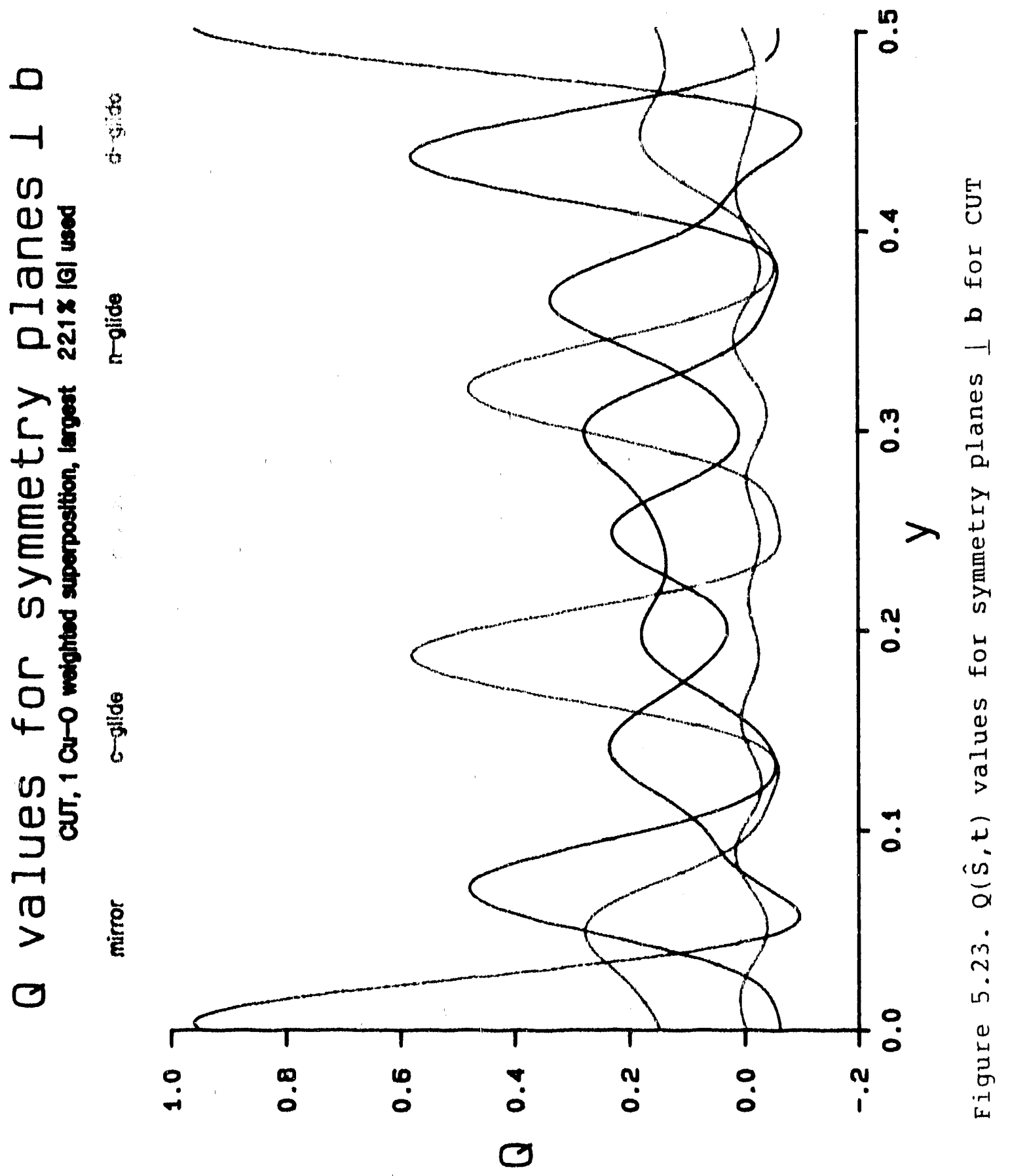


Table 5.16. Symmetry Operator Locations for $\mathrm{Cu}\left(\mathrm{C}_{6} \mathrm{O}_{3} \mathrm{H}_{9}\right) \mathrm{Cl}_{2}$

\begin{tabular}{ccccc}
\hline symmetry operator & $t^{a}$ & $Q(\hat{s}, t)$ & $s(\hat{S}, t)$ \\
$-x, y,-z$ & $(-.0005,0.0,0.0012)$ & 0.9004 & 0.0201 \\
$-x, y+1 / 2,-z$ & $(0.2495,00,0.0012)$ & 0.9004 & 0.0201 \\
$x,-y, z+1 / 2$. & $(0.0,0.1861,0.0)$ & 0.5820 & 0.0907 \\
$1 / 2+x,-y, 1 / 2+z$ & $(0.0,0.4361,0.0)$ & 0.5820 & 0.0907 \\
$-x,-y,-z$ & $(-.0005,0.0025,0.0013)$ & 0.8554 & 0.0293 \\
$-x,-y,-z$ & $(0.2495,0.2525,0.0011)$ & 0.8554 & 0.0293
\end{tabular}

arranslations are given in fractions of a unit cell.

generates an n-glide plane one quarter of the unit cell away from the c-glide plane, which is clearly indicated in the plot.

Even though there is a large amount of pseudo-symmetry remaining in the superposition map, when the $G_{h}$ are transformed, the phases of the $E_{h}$ are refined using the EG relationship and an electron density map is calculated, the $\mathrm{Cu}$ atom, both $\mathrm{Cl}$ atoms and all three $\mathrm{O}$ atoms are all found in the largest 9 peaks on the map. The $\mathrm{Cu}$ atom and the $\mathrm{Cl}$ atoms are the three largest peaks in the electron density map. The three other large peaks in map are pseudo-related to the $\mathrm{Cl}$ atoms and one of the oxygen atoms.

Two additional weighted superpositions were carried out using the $\mathrm{Cu}-\mathrm{Cl}$ vectors. The list of peaks in the superposition map was reduced using TRIM. The 300 highest peaks in the list were checked for c-glide-related peaks in the list (using a tolerance of $0.6 \mathrm{~A}$ ). The Fourier transform 

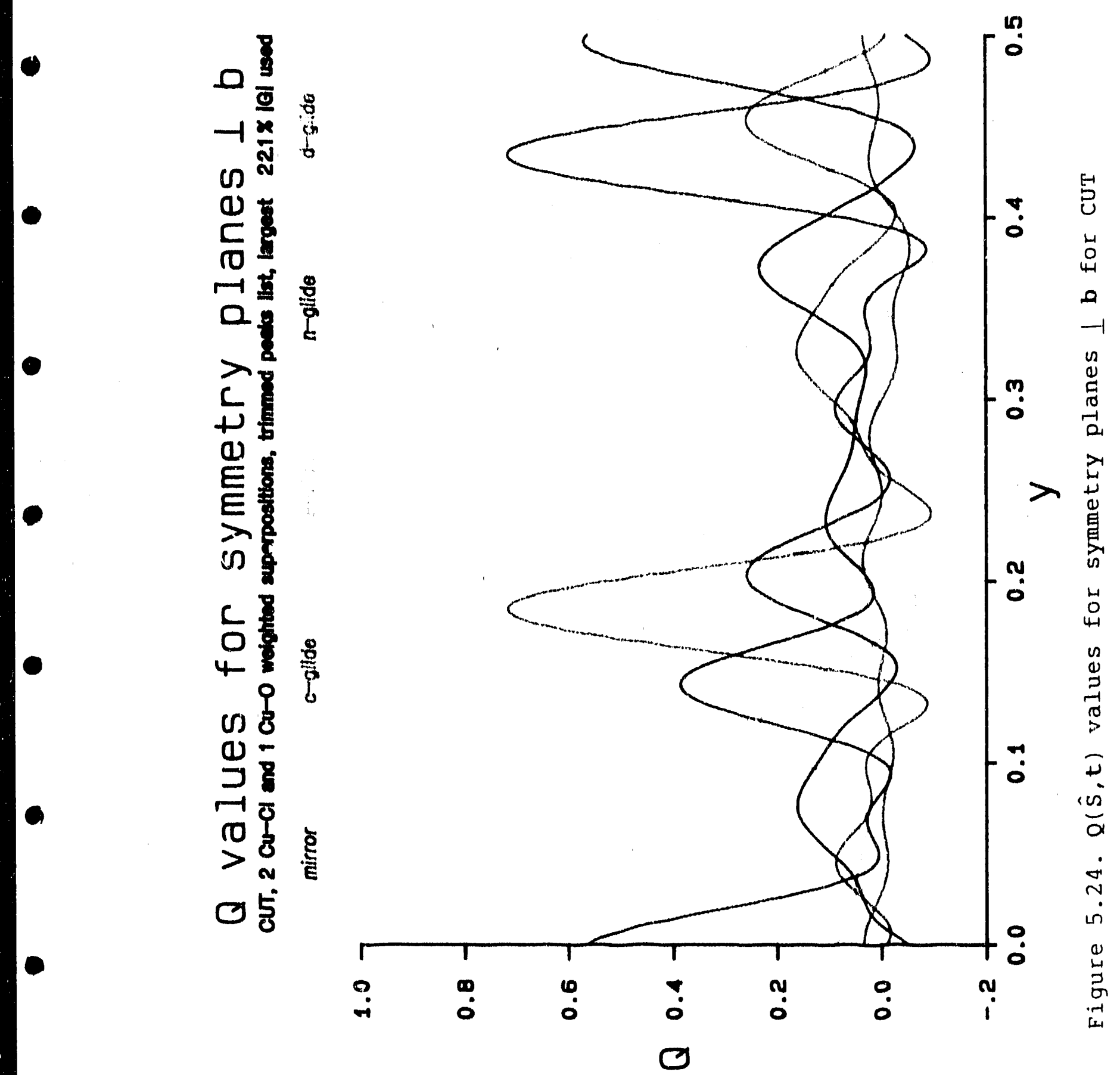
Table 5.17. HYPAD Atomic Coordinates ${ }^{a}\left(\times 10^{4}\right)$ for $\mathrm{Cu}\left(\left(\mathrm{C}_{6} \mathrm{O}_{3} \mathrm{H}_{9}\right) \mathrm{C}_{2}\right.$

\begin{tabular}{|c|c|c|c|c|c|}
\hline attom & $\#$ & pk.tht. & $x$ & $\mathbf{Y}$ & 2 \\
\hline $\begin{array}{l}C w \\
c 11 \\
c 12 \\
011 \\
02 \\
03 \\
c 1 \\
C 2 \\
c 3 \\
C 4 \\
c 5 \\
C 6\end{array}$ & $\begin{array}{r}11 \\
2 \\
3 \\
4 \\
9 \\
5 \\
116 \\
114 \\
110 \\
118 \\
112 \\
115\end{array}$ & $\begin{array}{r}264 \\
11.11 \\
10.7 \\
5.9 \\
39 \\
58 \\
2.4 \\
2.5 \\
37 \\
23 \\
27 \\
24\end{array}$ & $\begin{array}{r}-18 \\
1563 \\
-1355 \\
470 \\
988 \\
-1426 \\
338 \\
1303 \\
1045 \\
-505 \\
-1407 \\
-0.08\end{array}$ & $\begin{array}{r}1879 \\
276 \\
580 \\
1870 \\
4355 \\
3430 \\
3396 \\
4747 \\
5650 \\
6256 \\
4869 \\
4090\end{array}$ & $\begin{array}{r}-11 \\
818 \\
-2499 \\
2192 \\
782 \\
-672 \\
2779 \\
3026 \\
1954 \\
369 \\
160 \\
1674\end{array}$ \\
\hline
\end{tabular}
cell.

atomic coordinates are given as fractions of the unit Table 5.11:. Refined Atomic Coordinates ${ }^{a}\left(x 10^{4}\right)$ for $\mathrm{Cu}\left(\left(\mathrm{C}_{6} \mathrm{O}_{3}, \mathrm{Hig}\right)\right) \mathrm{Cl}_{2}$

\begin{tabular}{|c|c|c|c|}
\hline atom & $\mathbf{x}$ & $y$ & 2 \\
\hline $\begin{array}{l}\mathbb{C} 1 \\
\mathbb{C} 11 \\
\mathbb{C} 12 \\
011 \\
0.2 \\
0.3 \\
\mathbb{C} 11 \\
\mathbb{C} 2 \\
\mathbb{C} 3 \\
\mathbb{C} 4 \\
\mathbb{C} 5 \\
\mathbb{C} 6\end{array}$ & 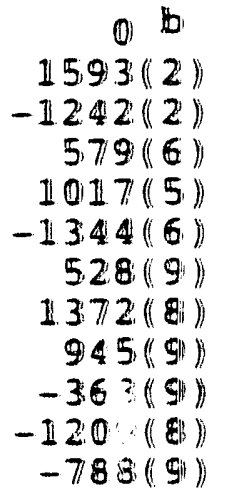 & $\begin{array}{r}1892(11) \\
225(4)) \\
5775(4) \\
1797(8)) \\
4366(18) \\
3395(9) \\
3475(113) \\
4872(14)) \\
5703(12) \\
6335(13) \\
4963(112) \\
4162(13)\end{array}$ & $\begin{array}{c}0 \\
869(3) \\
-2406(3) \\
2297(7)) \\
829(7) \\
-681(7) \\
2945(11) \\
3214(11) \\
1692(11) \\
557(12) \\
262(11) \\
1788(12)\end{array}$ \\
\hline
\end{tabular}

atomic coordinates are given as fractions of the unit cell.

bestimated standard deviations for the refined coordinates are given in parentheses for the least significant diitit. 
Table 5.19. Comparative bond distances for $\mathrm{Cu}\left(\mathrm{C}_{6} \mathrm{O}_{3} \mathrm{H}_{9}\right) \mathrm{Cl}_{2}$

\begin{tabular}{lccc}
\hline atoms & refine & HYPAD $(A)$ & $\Delta(A)$ \\
\cline { 3 - 4 }$C u-C 11$ & $2.243(3)$ a & 2.22 & -.02 \\
$C u-C 12$ & $2.232(3)$ & 2.30 & 0.07 \\
$C u-01$ & $2.151(6)$ & 2.10 & -.05 \\
$C u-02$ & $2.126(6)$ & 2.12 & -.01 \\
$C u-03$ & $1.949(7)$ & 2.05 & 0.10 \\
$01-C 1$ & $1.464(11)$ & 1.39 & -.07 \\
$02-C 3$ & $1.454(11)$ & 1.61 & 0.16 \\
$03-C 5$ & $1.493(11)$ & 1.41 & -.08 \\
$C 1-C 2$ & $1.497(14)$ & 1.62 & 0.12 \\
$C 1-C 6$ & $1.538(14)$ & 1.47 & -.07 \\
$C 2-C 3$ & $1.521(13)$ & 1.21 & -.31 \\
$C 3-C 4$ & $1.518(14)$ & 1.78 & 0.26 \\
$C 4-C 5$ & $1.476(14)$ & 1.59 & 0.11 \\
$C 5-C 6$ & $1.520(13)$ & 1.46 & -.06 \\
\hline
\end{tabular}

aEstimated standard deviations for the refined distances are given in parentheses for the least significant digit. 
Table 5.20. Comparative bond angles for $\mathrm{Cu}\left(\mathrm{C}_{6} \mathrm{O}_{3} \mathrm{H}_{9}\right) \mathrm{Cl}_{2}$

\begin{tabular}{|c|c|c|c|c|c|}
\hline \multicolumn{3}{|c|}{ atoms } & refined $\left({ }^{\circ}\right)$ & HYPAD $\left({ }^{\circ}\right)$ & $\Delta\left({ }^{0}\right)$ \\
\hline $\begin{array}{l}\text { Cl1 } \\
\text { Cl1 } \\
\text { Cl1 } \\
\text { Cl1 } \\
\text { Cl } 2 \\
\text { Cl2 } \\
\text { Cl2 } \\
01 \\
01 \\
02 \\
\text { Cu } \\
\text { Cu } \\
\text { Cu } \\
01 \\
01 \\
\text { C2 } \\
\text { C1 } \\
02 \\
02 \\
\text { C2 } \\
\text { C3 } \\
03 \\
0 \text { 1 } \\
\text { C4 } \\
\text { C5 }\end{array}$ & 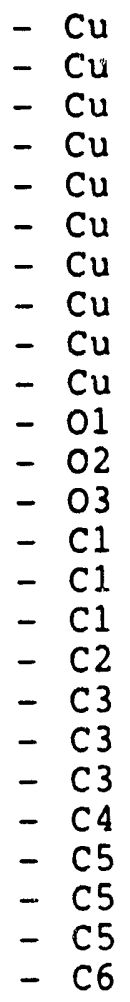 & $\begin{array}{l}-C 12 \\
-01 \\
-02 \\
-03 \\
-01 \\
-02 \\
-03 \\
-02 \\
-03 \\
-03 \\
-c 1 \\
-c 3 \\
-c 5 \\
-c 2 \\
-c 6 \\
-c 6 \\
-c 3 \\
-c 2 \\
-c 4 \\
-c 4 \\
-c 5 \\
-c 4 \\
-c 6 \\
-c 6 \\
-c 1\end{array}$ & $\begin{array}{r}92.86(11)^{a} \\
92.15(19) \\
91.59(18) \\
177.84(20) \\
143.15(18) \\
130.80(17) \\
89.28(20) \\
85.49(23) \\
86.30(25) \\
86.79(26) \\
119.10(50) \\
119.93(51) \\
124.02(51) \\
109.27(78) \\
109.00(74) \\
112.33(78) \\
114.32(78) \\
110.87(72) \\
106.75(72) \\
112.17(80) \\
114.45(80) \\
110.01(73) \\
107.03(69) \\
113.09(78) \\
112.69(78)\end{array}$ & $\begin{array}{r}94.3 \\
96.2 \\
90.7 \\
177.8 \\
141.7 \\
129.9 \\
87.9 \\
86.7 \\
82.5 \\
87.7 \\
122.3 \\
117.3 \\
128.9 \\
98.6 \\
112.9 \\
114.7 \\
122.5 \\
109.9 \\
91.0 \\
116.9 \\
113.3 \\
100.5 \\
106.3 \\
112.8 \\
117.8\end{array}$ & $\begin{array}{r}1.4 \\
4.0 \\
-.9 \\
0.0 \\
-1.5 \\
-.9 \\
-1.4 \\
1.2 \\
-3.8 \\
0.9 \\
3.2 \\
-2.6 \\
4.9 \\
-10.7 \\
3.9 \\
2.4 \\
8.2 \\
-1.0 \\
-15.8 \\
4.7 \\
-1.2 \\
-9.5 \\
-.7 \\
-.3 \\
5.1\end{array}$ \\
\hline
\end{tabular}

aEstimated standard deviations for the refined angles are given in parentheses for the least significant digits. 
coefficients of this new superposition map were deterinined. A plot of the $Q(\hat{S}, t)$ function for symmetry planes perpendicular to $b$ is shown in Figure 5.24. As can be seen in this plot, the amount of pseudo-symmetry has been reduced significantly. The $G_{h}$ were transformed and the phases of the $E_{h}$ that were refined using the EG relationship were used to calculate an electron density map. Figure 5.25 shows the projection of the electron density map onto the leastsquares plane. All of the non-hydrogen atoms were found in the largest 18 peaks in the map. The HYPAD atomic positions, peak number and peak height are given in Table 5.17 and the refined positions are given in Table 5.18. The bond distances and bond angles obtained from the HYPAD and the refined positions are listed in Tables 5.19 and 5.20 , respectively. 
CHAPTER 6. CONCLUSION

I hope that the results presented in Chapter 5 successfully show that HYPAD is a viable alternative to the existing automatic phase determining techniques. We have shown that for simple organo-metallics, complete structural solutions can be obtained and for more complex compounds, a sizable part of the structure can be obtained. It is encouraging to note that if the space group is not uniquely defined by the extinction conditions, HYPAD also appears to be able to correctly determine the space group.

Although the presence of spurious peaks in the superposition map can cause erroneous phase predictions, centrosymmetric structures seem to be less susceptible to this than do non-centrosymmetric structures. This is not unexpected, since there are only two phase choices for the structure factors of centrosymmetric structures. It is very unlikely that the spurious peaks will be in the correct oriertation to cause the phase to be shifted from $0^{\circ}$ to $180^{\circ}$ (or $180^{\circ}$ to $0^{\circ}$ ). It is more likely that the phase would be shifted by several degrees, say 20 or $30^{\circ}$, which could have a significant effect on the EG refinement in the noncentrosymmetric case.

There is a growing need for a procedure that can work as an alternative to direct methods. Very often, when direct methods fails, it is the result of a poor starting set of 
phases. The user who is inexperienced in the use of direct methods is faced with a dilemma when the first attempt fails. Usually, there is no obvious choice as to what changes to make in the starting set or in the phases initially assigned to these reflections. It has been shown that a good initial set of phases can be obtained from the transformed Fourier coefficients of the superposition map, and that these phases, when refined via the EG relation, can produce, in most cases, either the structure or a sizable fragment thereof.

Furthermore, if HYPAD fails in its first attempt, other superposition vectors can be chosen by using the location of the symmetry elements to locate symmetry-related peaks. The superposition map obtained using these vectors will generally provide a good starting set of phases. 
CHAPTER 7. FUTURE WORK

It should be realized that HYPAD is still in the developmental stage. A number of the structures selected up to this point were chosen to provide a means of testing the computer code rather than to fully explore the the capabilities of the method. There are still areas of the hybrid Patterson superposition-diract methods philosophy that need to be examined. It is to be fully expected that there will be structures that resist solution by this method. It would be a great benefit, then, to examine these types of structures to try to pinpoint at which aspect(s) of the current methodology difficulties are encountered.

One of the more obvious difficulties that is encountered is the presence of multiple images. As has been shown, weighted superpositions reduce this problem, but does not eliminate it. Since the $\xi(t)$ and $\xi^{\prime}(t)$ functions, as well as the $Q(\hat{s}, t)$ function, can be used to determine the location of one image relative to another, it would seem that it should be possible to use the $G_{h}$ from an unweighted superposition to gain information about a single image, but very little work has been done in this area.

In many of the structures which seem to be unsolvable, the space group symmetry is questionable. Patterson, and to a lesser extent, superposition maps contain additional symmetry which can further cloud the issue. While the 
results obtained concerning the determination of space group symmetry are very encouraging, the user must be conscious of the effects of pseudo-symmetry. The ultimate goal, then, would be the automatic determination of the space group by the program without use: input. Obviously, if this automation could be done, then the rest of the procedure could follow.

The routine could automatically shift the phases of the $\sigma_{h}$, assign and then refine the phases of the $E_{h}$. A quantity which would indicate how good the phases are, such as the rasidual index, vould be calculated and depending upon its value, one of two cholces would be made. If the phases were considered "good", the refined phases would be reintroduced into the $E G$ or $\Sigma_{2}$ relation to produce a new refined set of phases. The iterations of phase refinement would continue until the phase set has converged. If the phases were considered "poor", the routine would use the locations of the symmetry elements to automatically calculate the positions of atoms that are symmetry-related to the atoms in the shift vector used in the original superposition. These interatomic vectors would then be used as shift vectors in multiple superpositions. New Fourier coefficients would be calculated and the process restarted, like the approach used in the solution of $\mathrm{FeP}_{2} \mathrm{OC}_{32} \mathrm{H}_{31} \mathrm{I}$ described in Chapter 5 .

Ideally, the procedure would be automated to the point that the user would only need to indicate the peak to be 
used for the first superposition, and the procedure would take over, making all of the decisions, and outputing the solution. In fact, it might be possible to have the routine make the choice of the first superposition vector using the distance of the peak from the origin and the peak height as criteria for its decision.

One always has to worry that the EG relation may produce phases for the $E_{h}$ that resemble the superposition map more than they resemble the phases of the electron density map. It may turn out that it is best if both the EG and $\Sigma_{2}$ refinements are carried out in tandem. In the early stages of refinement, the phases obtained from the EG relationship would have more weight than those obtained from the $\Sigma_{2}$ relation, but as the refinement proceeded, the EG phases would decrease in importance and the $\Sigma_{2}$ phases would increase in importance. 
APPENDIX A. KEYWORD OPTIONS IN HYPAD

All of the user-created input files that are not created by the CHES.CAT procedure use a keyword format for the imput of parameters into the individual programs in HYPAD. This type of approach allows the user to easily see which parameters will be used, and greatly facilitates modification of the input files keywords for the individual programs are listed below.

only the first three letters of each keyword are required. In the following lists, the ktywords are given in uppercase letters and the type of value fo: the keyword (if any) is indicated by one of three codes: $n \Rightarrow$ integer number, $x \Rightarrow$ real (floating point) number, $c \Rightarrow$ character string. Some of the keywords have default values that the program will use if the keyword is missing; these are indicated in (]'s. Except in certain cases, it is possible to have more than one keyword (and its accompanying value) on a line. The keyword and its value(s) must be on the same line. Special cases when only one keyword can appear on a line are indicated in the description of the appropriate keyword. 
2THETA $\quad x$

LAUE $n$
PEAKS $n$

- the number of peaks to be read in from XXXSUPR.PKS and used to calculate the $G_{h}$. This number must be less than or equal to 200 , and if it is 0 , then the entire superposition map will be read in and transformed.

(Map option is not available at this time)

- the maximum $2 \theta$ value to use in

calculating the $G_{h}$.

WAVELENGTH $x$ - the wavelength of the radiation used.

- code indicating the Laue symmetry. The Laue symmetries and their corresponding codes are:

$1 \Rightarrow$ triclinic, -1 symmetry

$2 m$ monoclinic, b-unique, $2 / \mathrm{m}$ symmetry

$3 \Rightarrow$ monoclinic, c-unique, $2 /$ m symmetry

$4 \Rightarrow$ orthorhombic, mmm symmetry

$5 \Rightarrow$ tetragonal, $4 / m$ symmetry

$6 \Rightarrow$ tetragonal, $4 / \mathrm{mmm}$ symmetry

7 trigonal, -3 symmetry

$8 \Rightarrow$ trigonal, $-3 m$ symmetry

$9 \Rightarrow$ hexagonal, $6 / \mathrm{m}$ symmetry

$10 \Rightarrow$ hexagonal, $6 / \mathrm{mmm}$ symmetry

$11 \Rightarrow$ cubic, $-3 m$ symmetry 


\section{2}

$$
12 \Rightarrow \text { cublc, m3m symmetry }
$$

LATTICE C

- code indicating the centering of the lattice.

$$
\begin{aligned}
& P \Rightarrow \text { Primitive } \\
& A \Rightarrow A \text {-centered } \\
& B \Rightarrow \text { B-centered } \\
& C \Rightarrow \text {-centered } \\
& I \Rightarrow I \text {-centered (body centering) } \\
& F \Rightarrow \text {-centered (face centering) } \\
& R \Rightarrow R \text {-centered (rhombohedral) }
\end{aligned}
$$

UNKNOWN

- space group is unknown. If this keyword is included anywhere in the input file, the space group will be considered unknown, even if the KNOWN keyword appears later in the file.

KNOWN

- space group is known (inclusion of this is optional)

LOCSYM. INP

2 THETA $\times \times$

- the minimum and maximum $2 \theta$ values to use. All $G_{h}$ in this range will be used in the calculations.

LAUE $\mathrm{n}$ - code indicating the Laue symmetry. See above for the appropriate codes. 
WEIGHTS C

FRACTIONG

NSUPERPOS

PLOT C

UNWTSUPPOS

WTSUPERPOS

SPECIAL
- Indicates the weighting scheme to be used in calculating $s(\hat{s}, t)$. "c" must bo one of the cholces given below, no abbreviations are allowed.

$$
\begin{aligned}
& \text { UNIT } \Rightarrow\left|G_{h} G_{h}\right| \text { weights } \\
& E(h) \Rightarrow\left|E_{h} G_{h} G_{h}\right| \text { weights }
\end{aligned}
$$

$x$ - the fraction of the $G_{h}$ to be used in

$$
\text { calculating } Q(\hat{S}, t) \text { and } S(\hat{S}, t) \text {. }
$$

$n$ - the number of superpositions that were done.

- indicates that graphics output is

desired. The commands required to draw the plots of the $Q(\hat{S}, t)$ values for the symmetry planes will be written out.

$$
H P \Rightarrow H P-G L \text { commands }
$$

- Indicates that unweighted superpositions were done and that the "mirrored" form of $S(\hat{S}, t)$ will be calculated.

- indicates that weighted superpositions were done (inclusion of this is optional as it is the default condition).

- If the user suspects that the tail atom of the superposition (1.e., the one at the origin) actually lies on a special position, then this option should be used. The figure-of-merit approach sometimes 
passes over a valid translation cholce because the program thinks that it is a result of the superpostion failing to completely remove the Patterson symmetry. This option will save the two "best" translations for later analysis.

SYMMETRY

- Indicates that the rest of the file conialns symmetry operations. Therefore, it MUST be the last keyword in the 11 st. It must stand alone ( $1 . e .$, do not include a symmetry operator on the same line because the operator will be ignored and do not include it on the same line with other keywords as they will also be ignored).

The remaining lines are formatted $(A 1, A 26)$, and contain the variables COPT and CSYMM (if COPT is a \#, then the following line is free-format).

COPT

- a special option indicator (blank) $\Rightarrow$ no special option * $\Rightarrow$ space group symmetry operator check is desired, only works for symmetry planes 
$\# \Rightarrow$ the transiation for which the symmetry operator is to be evaluated follows.. The next Iine is a free format line containing the $X, Y, z$ coordinates of the transllation.

CSYMA - the symmetry operator in alpha-numeric form.

MULTIIM. IMP

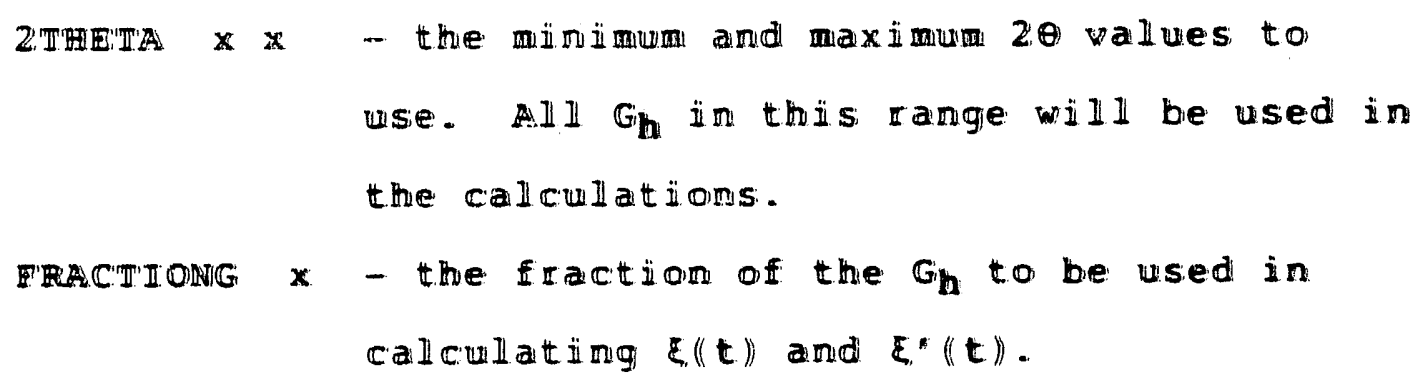

FOURIER . INP

MAP I

- indicates which type of Fourier map should be callculated. Map type 3 is the usval choice for the rour routine. The map types and theil codes are:

$$
\begin{aligned}
& 1 \Rightarrow \text { Patterson, using }\|\mathbf{F}\| \\
& 2 \Rightarrow \text { Patterson, using }\|\mathbf{F}\| \\
& 3 \Rightarrow \text { Ellectrom density. using Fhubs) }
\end{aligned}
$$


$4 \Rightarrow$ Difference electron density, using

$F_{h}($ obs $)-F_{h}(\mathrm{calc})$

$5 \Rightarrow$ Electron density, using $F_{h}(c a l c)$

$6 \Rightarrow$ Electron density, using

$A_{h}(c a l c) * F_{h}(o b s)^{2}$ and $B_{h}(c a l c) * F_{h}$ (ovs $^{2}$

$7 \Rightarrow$ Electron density, using

$A_{h}(c a l c) * F_{h}(o b s)$ and $B_{h}(c a l c) \star F_{h}(o b s)$

ORIENT! $n$

- the map orientation. HYPAD requires

that orientation 2 be used.

$\begin{array}{rrrrr} & \text { across } & \text { down } & \frac{\text { layers }}{1} \\ 1 \Rightarrow & x & y & z \\ 2 & x & z & y \\ 3 & \Rightarrow & y & x & z \\ 4 & \Rightarrow & y & z & y \\ 5 & \Rightarrow & z & x & y \\ 6 & \Rightarrow & z & y & x\end{array}$

FIELDWIDTH $n$ - the number of columns of printed output for each grid point.

$$
\begin{aligned}
& 2 \Rightarrow 2 \text { columns, base } 10 \\
& 3 \Rightarrow 3 \text { columns, base } 10 \\
& 4 \Rightarrow 4 \text { columns, base } 10 \\
& 6 \Rightarrow 2 \text { columns, base } 36(0-9, A-2) \\
& 7=3 \text { columns, base } 36
\end{aligned}
$$

LOWVALUE $n$ - the minimum value printed on the map 
LAUE $n$

BLANKS $\mathrm{n}$

LAYER $n \mathrm{n}$
- Laue symmetry of the crystal. NOTE!!!

This is a different Laue code than what is used in t.le rest of the package.

$1 \Rightarrow-1$ symmetry, triclinic

$2 \Rightarrow 2 / m$ symmetry, monoclinic

$3 \Rightarrow$ mmm symmetry, orthorhombic

$4 \Rightarrow-3$ symmetry, trigonal

$5 \Rightarrow-3 m$ symmetry, trigonal

$6 \Rightarrow 4 / m$ symmetry, tetragonal

$7 \Rightarrow 4 / \mathrm{mmm}$ symmetry, tetragonal

$8 \Rightarrow 6 / m$ symmetry, hexagonal

$9 \Rightarrow 6 / \mathrm{mmm}$ symmetry, hexagonal

$10 \Rightarrow m 3$ symmetry, cubic

$11 \Rightarrow m 3 m$ symmetry, cubic

- the number of blank lines between

printed output lines

GRID $n \mathrm{n} n$

- the number of grid points along the 3

crystal axes. The first number of the

number of grids along $a$, the second is the

number of grids along $b$, and the last is

the number of grids along $c$. The allowed

values re $0,16,32,64,128$.

- the minimum and maximum layers to be

calculated. The minimum must be $\geq 0$ and

the maximum must be $\leq$ the number of grids

in the layer direction. 


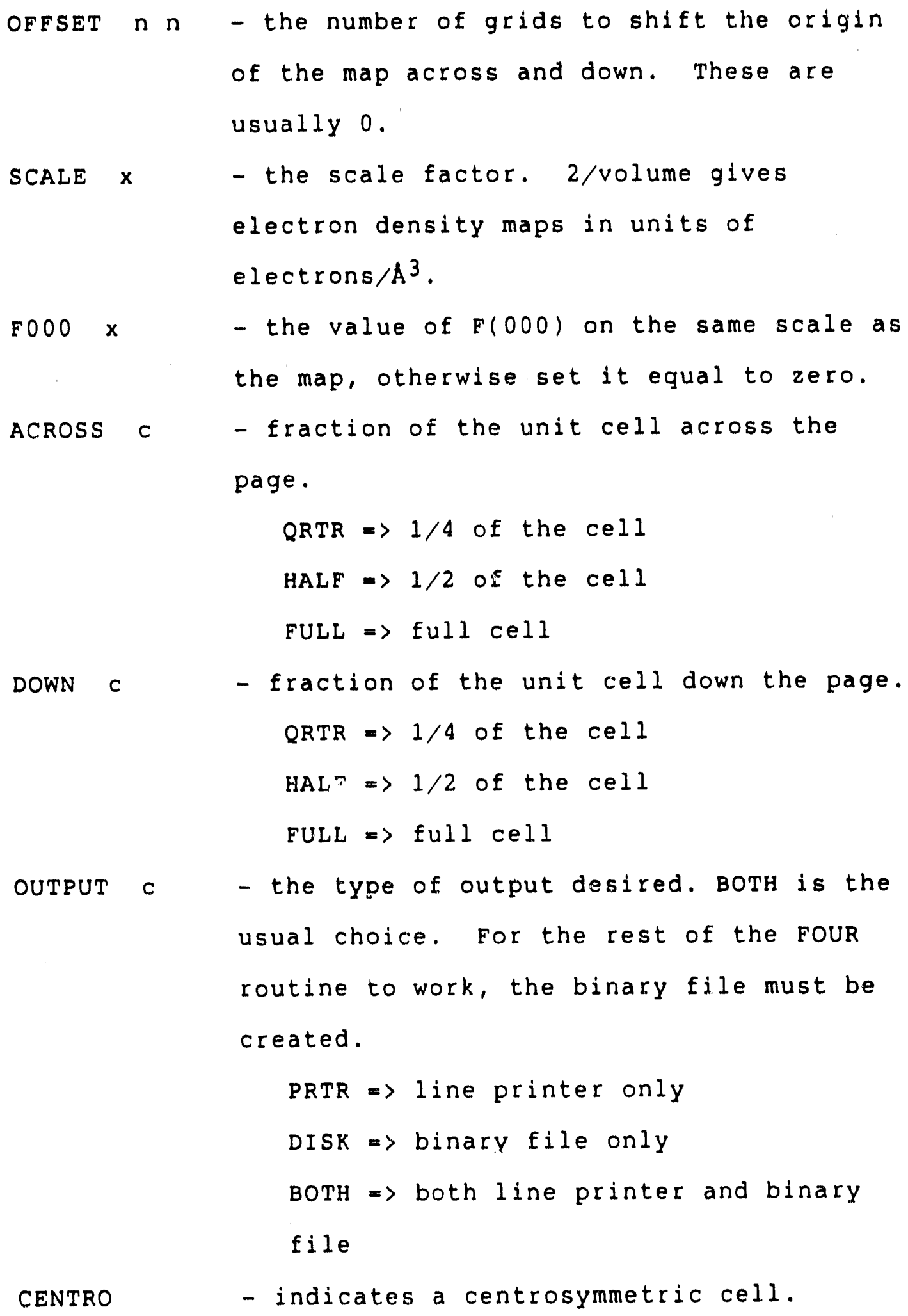


NONCENTRO

LATTICE C

SYMMETRY $n$

- Indicates a non-centrosymmetric cell.

- indicates the lattice symmetry

$$
\begin{aligned}
& P \Rightarrow \text { Primitive } \\
& A \Rightarrow \text { A-centered } \\
& B \Rightarrow \text { B-centered } \\
& C \Rightarrow \text {-centered } \\
& F \Rightarrow \text { Face-centered } \\
& I \Rightarrow \text { Body-centered } \\
& R \Rightarrow \text { Rhombohedral }
\end{aligned}
$$

- the number of symmetry elements, excluding the center of symmetry and the lattice symmetry operations. This must be the last keyword in the list, as the program assumes that all lines following this contain symmetry operators in alphanumeric form $(X, 1 / 2+Y, Z)$, one per line. This keyword must also be on a line by itself, any other keywords on this line will be ignored.

\section{PRESEARCH. INP}

The first line of the file must be the title ( 80 characters maximum). It is primarily used as a header for the SEARCH output. The following lines in the file contain keywords or symmetry elements. 
ATOMS $\mathrm{n}$

ANOMALOUS $n$

SYMMETRY $n$
- the number of atoms in the asymmetric unit, $\geq 1$.

- the number of types of atoms with significant anomalous scattering effects. Usually, these are atoms whose atomic numbers are greater than 9.

CELL $x \times x \times x \times$ - the unit cell lengths $a, b, c$ (in $A$ ) and the angles alpha, beta, gamma (in degrees).

- the number of symmetry elements, excluding the center of symmetry and the lattice symmetry operations. This must be the last keyword in the list, as the program assumes that all lines following this contain symmetry operators in alpha-numeric form $(X, 1 / 2+Y, Z)$, one per line. This keyword must also be on a line by itself, any other keywords on this line will be ignored.

SEARCH. INP

This routine is part of MULTAN80. The list of keywords are included here as a convenience. If the first card is 
blank, defaults will be assumed throughout.

PEAKS $n$

ATOMS $n$

NOJOIN
- $n$ is the number of peaks to be searched for in the map. The default is $(11 * n) / 9$ where $n$ is the number of independent atoms (excluding hydrogens). If two clusters are within $2.8 \mathrm{~A}$ of each other, the default is automatically increased to $\left(3 *^{*}\right) / 2$.

- $n$ is the number of additional atoms input by the user to be included in the interpretation of the map (number of peaks + number of additional atoms must be less than 200). See below.

- requests that no interpretation of the peaks be done. The program prints a list of the highest peaks and then stops. This condition is set automatically if no chemical bonds can be found by the program.

PROJECTIONS $n$ - the number of orthogonal projections of each cluster to be plotted (to a maximum of three). The default is to always plot the least-squares projection, and to plot the projection orthogonal to the leastand most-squares planes if the cluster is cylindrical or spherical. $n$ equal to zero 
suppresses all plotting.

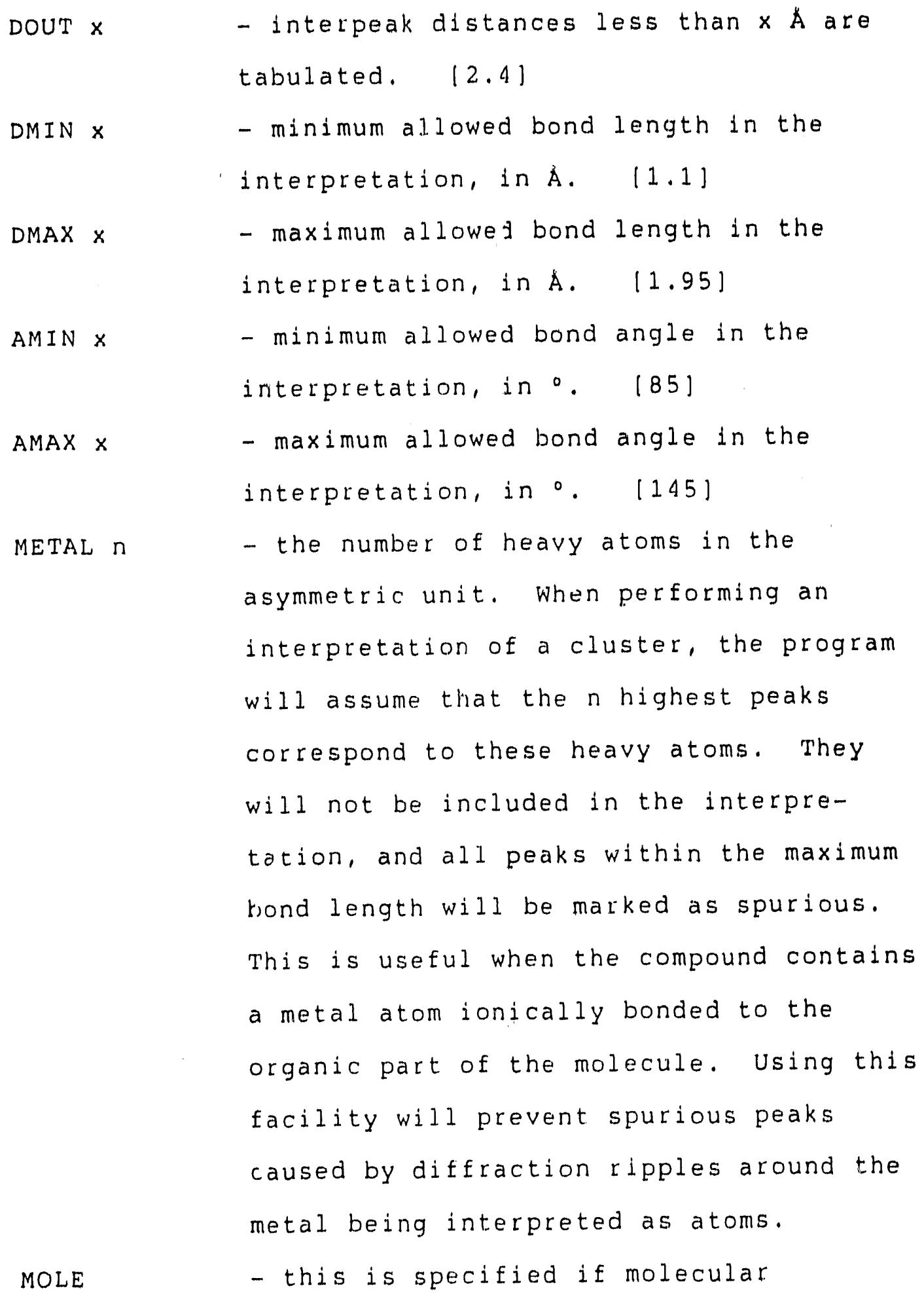


connectivity is to be input by the user (see below).

END

- this terminates the free format read and must be present if any keyword has been specified.

If 'ATOMS' is specified, the card following the one containing 'END' has the run time format for reading the $n$ sets of atomic coordinates (e.g., (3F10.5)). Then follow $n$ cards with the atomic coordinates in that format. This facility will normaliy be used when a difference fourier is being interpreted. The inclusion of the subtracted atoms ensures that they are included in the bond-lengths and angles calculations, and also that they appear in the lineprinter picture of the structure.

If 'MOLE' is specified, free format cards after the 'END' keyword, (and after the atom cards above if they are present), specify the molecular connectivity. The bond codes allow the program to compare the molecular fragment it finds with that input here. They are not used by the program in its search for a fragment and are therefore optional.

The bond codes for any molecule are found as follows: (a) Draw the molecule or fragment whose chemical structure (not necessarily geometry) is known. 
e.9.1<smiles></smiles>

(b) Number the atoms in any order

e.g.1<smiles></smiles>

(c) Specify the connections to each atom as follows: $\begin{array}{llllllllllllllllll}1 & 3 & 4 / 2 & 3 & 6 & 7 / 3 & 1 & 2 / 4 & 1 & 5 / 5 & 4 & 7 / 6 & 2 & 8 & 9 / 7 & 2 & 5 / 8 & 6\end{array}$ $10,9610 / 1089 / 1$

which means atom 1 is bonded to atoms 3 and 4,2 is bonded to 3,6 and 7, etc.

Redundant information may be removed from the bond sequence

e.g., $134 / 2367 / 4 \quad 5 / 57 / 6 \quad 8 \quad 9 / 8 \quad 10 / 9 \quad 10 / 1$

or $\quad 134 / 2367 / 547 / 8610 / 9610 / 1$

in which each bond is specified only once.

(d) Type any of these descriptions in free format, ending the information with / The maximum rumber of atoms in the molecule is 200 . Up to five molecules can be input in this way and the complete data is terminated with ///

QPLANES. INP

It is possible for the program to generate more than one plot per run (simply by having more than one SYMMETRY 
keyword), but all non-syMMETRY keyworda after the firet SYMMETRY keyword w11 be ignored (1.e.., if the first plot is drawn in color with solid lines, so will the rest of thein). 2 THETA $x x$ - the minimum and maximum $2 \theta$ values. Only the $G_{h}$ which lie in this range will be used to calculate $Q(\hat{s}, t) . \quad(m i n=0,0$, $\max =50.01$

FRACTIONG $x$ - the fraction of the largest $\left|G_{h}\right|$ to be used in calculating $Q(\hat{S}, t)$. (1.0)

COLOR - Indicates colors are to be used to differentiate between the symmetry planes. This is the default cholce and inclusion of this keyword implies that the curves will be drawn using solid lines. The colors and the corresponding symmetry planes are:

$$
\begin{array}{ll}
\text { mirror } & \Rightarrow \text { red (pen 2) } \\
b / c / a-g l i d e & \Rightarrow \text { green (pen 3) } \\
c / a / b-g l i d e & \Rightarrow \text { yellow (pen } 4 \\
n-g l i d e & \Rightarrow b l u e ~(p e n ~ 5) \\
d \text {-glide } & \Rightarrow \text { orange (pen 6) }
\end{array}
$$

BLACK

- indicates that all curves are to be drawn as black lines. Inclusion of this keyword implies that the the curves will be drawn using different Inestyles. 
using different ino types. The line types and the corresponding symmetry planes ares

$$
\begin{array}{ll}
m i l i o r & \Rightarrow \text { solid } \\
b / c / a-g l i d e & \Rightarrow \text { dashed } \\
c / a / b-g l i d e & \Rightarrow d a s h-d o \\
n-g l i d e & \Rightarrow l o n g d a s h-s h o r t \text { dash } \\
\text { d-glide } & \Rightarrow \text { long dash-short dash- } \\
\text { short dash } &
\end{array}
$$

SOLID

- Indicates that the curves will be drawn using solid lines. This is the default cholce.

SYMMETRY C - the axis to which the symmetry planes are perpendicular.

\section{QROTLINE. INP}

$$
\begin{aligned}
& \text { 2THETA } x x \text { - the minimum and maximum } 2 \theta \text { values. Only } \\
& \text { the } G_{h} \text { which lie in this range will be } \\
& \text { used to calculate } Q(\hat{s}, t) \text {. } \quad(\mathrm{min}=0.0 \text {, } \\
& \max =50.01 \\
& \text { FRACTIONG } x \text {.. the fraction of the largest }\left|G_{h}\right| \text { to be } \\
& \text { used in calculating } Q(\hat{S}, t) .(1.0) \\
& \text { SYMME'TY C - the symmetry operator for which the } \\
& Q(\hat{s}, t) \text { values are to be calculated. } \\
& (-X, Y,-Z)
\end{aligned}
$$


FIX C

LOCATION $x$

SHITT $x$
- Indicates which coordinate is to be kept fixed. I t if rotation axis is paraliel to a or b, y le parallel to cl

- Indicates the value of the fixed

coordinate. $\quad(0.25)$

- Indicates how much the varying

coordinate will be shifted. Thia option is usualiy not used. $\quad(0.0)$

QSPLOI' INP

It is possible for the program to generate more than one plot per run (almply by having more than one SYMMETRY keyword), but all non-SYMMETRY keywords after the first SYMMETRY keyword wil1 be ignoled (1.e., if the first plot uses $30 \%$ of the largest $\left|G_{h}\right|$, so will the rest of them).

2THETA $x \quad x$ - the minimum and maximum $2 \theta$ values. Only the $G_{h}$ which lie in this range will be used to calculate $Q(\hat{S}, t)$ and $s(\hat{S}, t)$. $[\min =0.0, \max =50.0]$

TRACTIONG $x$ - the fraction of the largest $\left|G_{h}\right|$ to be used in calculating $Q(\hat{S}, t)$ and $s(\hat{s}, t)$. $[1.0]$

SYMMETRY C - the symmetry operator for which tho $Q(\hat{S}, t)$ and $S(\hat{S}, t)$ values are to be 


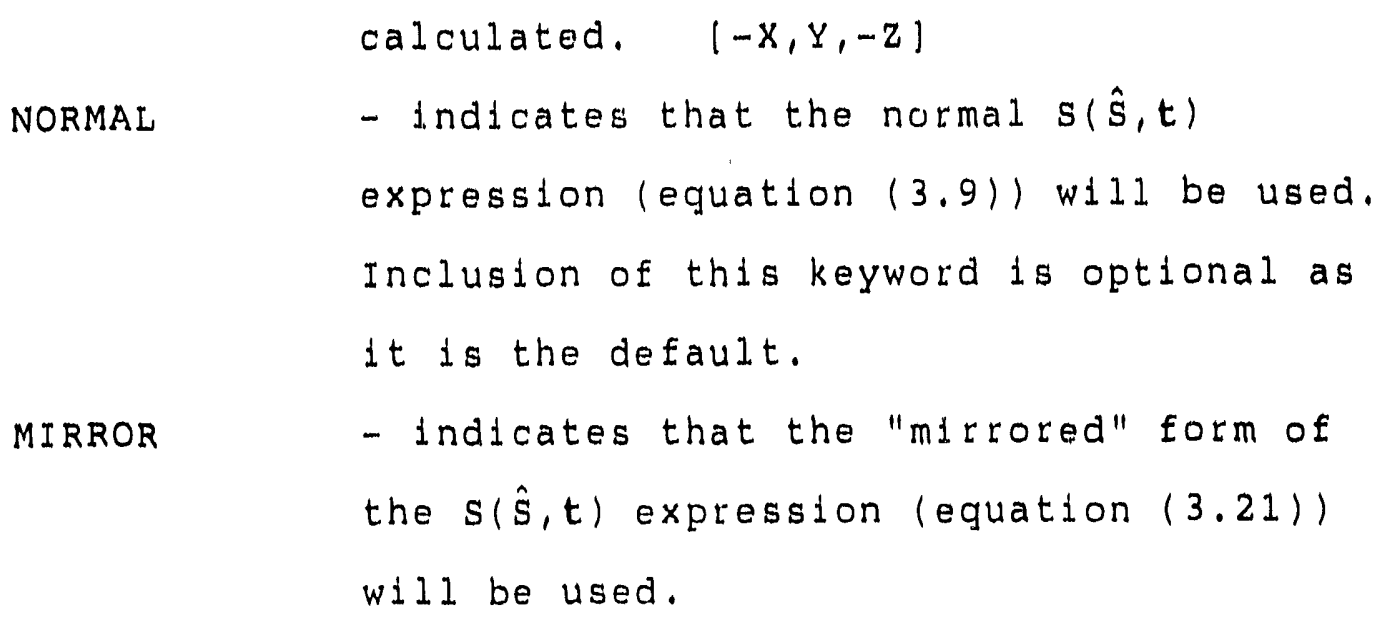

QDATA.INP

It is possible for the program to generate more than one data set per run (simply by having more than one sYMMETRY keyword), but all non-SYMMETRY keywords after the first SYMMETRY keyword will be ignored (1.e., if the first plot uses $30 \%$ of the largest $\left|G_{h}\right|$, so will the rest of them).

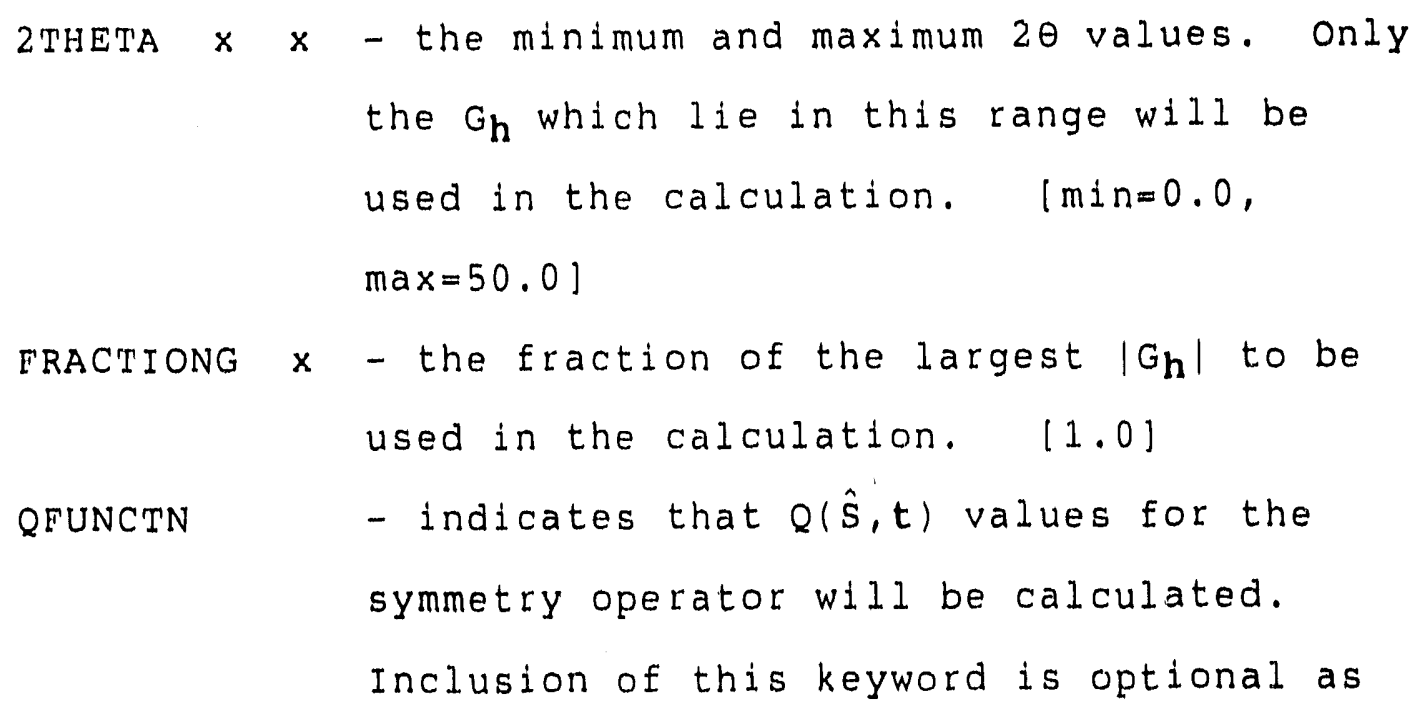


iit is the deffault.

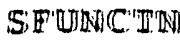

NORMLA D

MII $\mathbb{R} R(O)$

STMAMETTRY C
- inulicates that $S(\hat{S}, t)$ vallues for the symmett Iy operator il 11 be callewated.

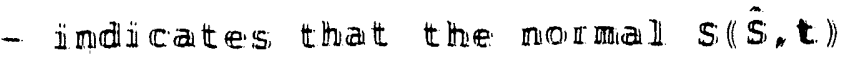
expression (equation $(3,9))$ mil 11 be wsed.

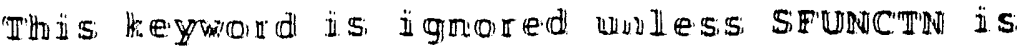

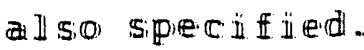

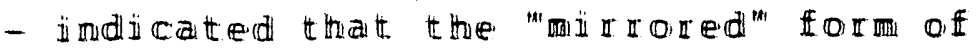
the $S\left(\vec{S}_{n}\right.$ th) expression ((equation $\left.(3.21)\right)$

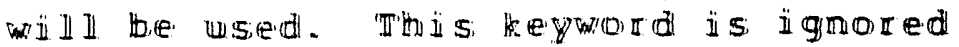

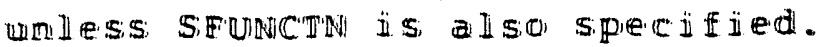

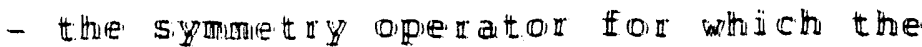
values are to be calcullated. $\left\|-\mathbb{Z}_{n} \mathbb{Y}_{n}-\mathbb{Z}\right\|$

Simple Imput Eli Iles

1

Siampile imputt fi lies, ffor two of the programs, LOCSYM and FOUUR, are Iisted bellow. This were wsed in the soliution of

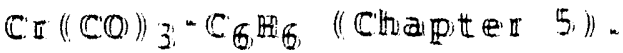

LOCSTM INP

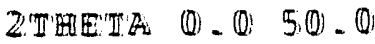

LAUUE: 2.

WEIGHTS UTNIT' FRACTIONG O. 3010

NSTUPERPS I WTSUPERPOS 
130

SYMMETRY

$-X, Y+1 / 2,-Z$

$\star X,-Y, Z$

$-X,-Y,-Z$ did on b

inv. diag (mirror) on b

inversion center

FOURIER. IN

CENTRE MAP 3 ORIENT" 2

OUTPUT BOTH GRID $3264 \quad 32$

ACROSS FULL DOWN FULL LAYER O 37

FIELDWIDTH 4 BLANKS O LOWVALUE 1

LAUE 2 SCALE $0.45634 \quad$ F000 0.000 OFFSET 00

LATTICE $\mathbb{P}$

SYMMETRY 2

$X, Y, Z$

$-X, 1 / 2+Y,-z$ 
Although the major emphasis of this research has been involved with the development of a new structural solution strategy, crystallographic studies of several compounds have been undertaken. This rppendix contains detailed discussions of these studies.

Structure Determination of $\left(\mathrm{NHC}_{5} \mathrm{H}_{5}\right)_{\mathrm{SbI}_{4}}$

\section{Introduction}

The crystal structure of pyridinium antimony(III) tetraiodide, $\left(\mathrm{NHC}_{5} \mathrm{H}_{5}\right)_{\mathrm{SbI}}$, has been determined as part of an ongoing series of investigations into halo-coordinated antimony compounds. Previously, the structures of chloroand bromo-coordinated single- and mixed-valence state antimony anions in the presence of various cationic species had been determined using $x$-ray diffraction. In these structures, the antimony ions coordinated to the halogens exist at the center of octahedral units or units best described as fragments of an octahedron 38-50. In some of the compounds, the trihalide and halide ions, as well as the halogen molecule, coexist in the crystal with the antimonycentered units, forming a bridge between the separated antimony-halide units $41,43,44,47$. However, this compound, 
like its chloro- and bromo-analogues 39,46 , forms infinite chains involving the $\mathrm{SbI}_{4}^{-}$anion via halide linkages.

\section{Experimental}

Crystal Data $\left(\mathrm{C}_{5} \mathrm{H}_{5} \mathrm{NH}\right) \mathrm{SbI}_{4}, \mathrm{M}=709.48$, orthorhombic, a $=25.156(6), b=13.960(2), c=7.838(1) \AA, V=2752.5 \AA^{3}$, space group pbca from systematic absences $0 k 1: k=2 n+1$, $h 0 l: l=2 n+1$ and $h k 0: h=2 n+1, D_{C}=3.42 \mathrm{~g} \mathrm{~cm}^{-3}, z=8, \mu(M O)=$ $108.6 \mathrm{~cm}^{-1}$.

Preparation $3.48 \mathrm{~g}$ of $\mathrm{SbI}_{3}(\mathrm{~s})$ were dissolved in 16.92 $\mathrm{ml}$ of concentrated $\mathrm{HI}(\mathrm{aq}) .1 .0 \mathrm{ml}$ of pyridine was added to the solution producing a thick black precipitate. The mixture was vacuum filtered and the precipitate was dried overnight in a desiccator using $40 \mathrm{ml}$ of concentrated $\mathrm{H}_{2} \mathrm{SO}_{4}$ as the desiccant. The precipitate was recrystallized in hot concentrated HI (aq), allowed to cool overnight and then placed in an ice bath for several hours until orange crystals had formed.

Crystallographic studies For data collection, a crystal of approximate dimensions $0.20 \times 0.20 \times 0.15 \mathrm{~mm}$ was mounted in a $0.2 \mathrm{~mm}$ diameter thin-walled capillary tube, sealed with wax, and attached to a standard goniometer head. The crystal was centered on a four-circle DATEX X-ray diffractometer. Four preliminary w-oscillation photographs were taken at various $\phi$ settings. The approximate positions of 12 reflections were selected from photographs and used as 
input to an automatic indexing program51. The resulting reduced cell and reduced cell scalars indicated a primitive orthorhombic lattice. The predicted layer spacings for this cell were observed, within experimental error, on three axial w-oscillation photographs.

Intensity data were collected at room temperature $\left(20^{\circ} \mathrm{C}\right)$ using Mo $K_{\alpha}(\lambda=0.71069 \AA)$ raciation on the above mentioned diffractometer equipped with a graphite monochromating crystal and interfaced to a VAX 730 computer in a real-time mode. A total of 2846 intensities were measured, corresponding to reflections in octants hkl and hkJ, using an w-step scan technique with a scan half-width of 0.5 degree. The data were coilected to a maximum $\sin \theta / \lambda$ of 0.5 ; an additional shell of data with $0.5<\sin \theta / \lambda \leq 0.6$ was collected but was found to be quite weak on the average, and was not used in the final refinement of the structure (see below). As a check on the stability of the crystal, the intensities of seven standard reflections were measured every 50 reflections during data collection. No significant decay was observed.

Accurate unit cell parameters were obtained by carefully centering 25 independent reflections, having $16^{\circ} \leq 2 \theta \leq 33^{\circ}$, on the diffractometer and inputing the $\pm 2 \theta$ values of these reflections into a least-squares fitting program. Intensity data were corrected for absorption $\left(\mathrm{T}_{\mathrm{min}} / \mathrm{T}_{\max }=\right.$ $0.045 / 0.082)$, as well as for Lorentz-polarization effects. 
of the 1886 reflections measured having $\sin \theta / \lambda \leq 0.5$, the 1039 that had $I \geq 3 \sigma(I)$ were considered to be observed. The symmetry equivalent reflections were averaged $\left(R_{a v}=0.049\right)$, yielding 607 reflections which were used in the refinement of the structure.

\section{Solution and Refinement}

The position of the antimony atom was determined from analysis of a sharpened patterson map. The positions of the remaining non-hydrogen atoms were determined from successive structure factor 52 and electron density map calculations 53 . The positional and isotropic thermal parameters of the antimony and iodine atoms were initially refined using a block-matrix least-squares procedure. The anisotropic thermal parameters of the antimony and iodine atoms, the isotropic thermal parameters of the pyridine ring, and the positional parameters of all the non-hydrogen atoms were refined to their final value using a full-matrix leastsquares procedure, minimizing the function $\Sigma w\left(\left|F_{O}\right|-\left|F_{C}\right|\right)^{2}$ to a conventional residual index of $R=\left(\Sigma|| F_{O}|-| F_{C}||\right) / \Sigma\left|F_{O}\right|=$ 0.039 and $a$ weighted residual index of $R_{W}=|\Sigma W|\left|F_{0}\right|-$ $\left.\left.\left|F_{C}\right|\right)^{2} / \Sigma w\left|F_{O}\right|^{2}\right]^{1 / 2}=0.039$, where $w=1 / \sigma^{2}(F)$. The positions of the hydrogen atoms were calculated and not refined. A secondary extinction correction was applied and gave a coefficient of $\gamma=5.303 \times 10^{-6}$. The final positional and averaged isotropic thermal parameters, with 
their standard deviations, of the $\mathrm{SbI}_{4}^{-}$anion and the pyridinium ring are given in Tables B.1 and B.2, respectively. The final anisotropic thermal parameters of the SbI4 $4^{-}$anton are given in Table B.3, and bond distances and angles are given in Table B.4. Retinement was also carried out with inclusion of data with $\sin \theta / \lambda>0.5$, but did not give improved standard deviations of the parameters $(R=0.049)$.

\section{Discussion}

The environment of the antimony atom in $\left(\mathrm{C}_{5} \mathrm{H}_{5} \mathrm{NH}\right) \mathrm{SbI}_{4}$ is shown in Figure B.1; infinite chains are formed similar to those found in the chloro and bromo analogues, even though the crystal system of the iodo compound is orthorhombic and the chloro and bromo compounds crystallize in the monoclinic system. A view of several unit cells is shown in Figure B.2. Each antimony lies at the center of a distorted octahedral structure, with all of the $I-S b-I$ bond angles within five degrees of $90^{\circ}$. The $\mathrm{Sb}-\mathrm{I} 3$ and $\mathrm{Sb}-\mathrm{I} 4$ bond distances, $2.809(4) \AA$ and $2.828(4) \dot{A}$ respectively, are slightly longer than the $2.67 \mathrm{~A} \mathrm{Sb}-1$ bond distances found in $\mathrm{SbI}_{3}$ by electron diffraction techniques ${ }^{38}$. I 1 and 12 are bridging iodines, connecting neighboring sbI4- moieties to form infinite chains via somewhat asymmetric bridges. The shorter Sb-I 1 and Sb-I2 bridging distances are $3.021(4)$ and 
Table B.1. Positionala $\left(\times 10^{4}\right)$ and Averaged Isotrople Thermal $\left(\mathrm{A}^{2}, \times 10^{3}\right)$ parameters for $\left(\mathrm{C}_{5} \mathrm{H}_{5} \mathrm{NH}\right) \mathrm{SbI}_{4}$

\begin{tabular}{crrrr}
\hline atom & $x$ & $y$ & $z$ & $U_{a v}{ }^{b}$ \\
\hline sb & $1268(1) c$ & $1614(1)$ & $-7978(3)$ & 57 \\
I1 & $1954.6(9)$ & $1700(2)$ & $-4820(3)$ & 73 \\
I2 & $565.1(9)$ & $1641(2)$ & $-11129(3)$ & 68 \\
I3 & $1976(1)$ & $245(2)$ & $-9290(3)$ & 84 \\
I4 & $579(1)$ & $231(2)$ & $-6544(3)$ & 84 \\
\hline
\end{tabular}
cel1.

apositional parameters are given as fractions of a unit

$b_{U_{a V}}=\left(U_{11}+U_{22}+U_{33}\right) / 3 . \quad T=\exp \left[-2 \pi^{2}\left(U_{11} h^{2} a^{* 2}+\right.\right.$ $u_{22} k^{2} b^{\star 2}+U_{33} l^{2} c^{\star 2}+2 U_{12} h k a^{\star} b^{\star}+2 U_{13} h l a^{\star} c^{\star}+$ $\left.2 U_{23} k 1 b^{*} c^{*}\right) 1$.

CEstimated standard deviations are given in parentheses for the least significant digits.

Table B.2. Positionala $\left(x 10^{4}\right)$ and Isotropic Thermal $\left(\mathbb{A}^{2}\right.$, $\times 10^{3}$ ) Parameters for the cation in $\left(\mathrm{C}_{5} \mathrm{H}_{5} \mathrm{NH}\right) \mathrm{SbI}_{4}$

\begin{tabular}{|c|c|c|c|c|}
\hline atom & $x$ & $y$ & $z$ & $u^{b}$ \\
\hline $\begin{array}{l}\mathrm{N} \\
\mathrm{C} 2 \\
\mathrm{C} 3 \\
\mathrm{C} 4 \\
\mathrm{C} 5 \\
\mathrm{C} 6\end{array}$ & $\begin{array}{l}1083(13) \mathrm{C} \\
1567(17) \\
1669(16) \\
1332(18) \\
861(19) \\
746(15)\end{array}$ & $\begin{array}{r}-791(22) \\
-975(28) \\
-1945(28) \\
-2605(25) \\
-2328(33) \\
-1398(25)\end{array}$ & $\begin{array}{l}-12564(37) \\
-13148(53) \\
-13146(53) \\
-12670(50) \\
-12073(60) \\
-11984(48)\end{array}$ & $\begin{array}{l}89(11) \\
89(12) \\
87(12) \\
88(12) \\
119(17) \\
77(11)\end{array}$ \\
\hline
\end{tabular}
cell.

apositional parameters are given as fractions of a unt

$b_{T}=\exp \left[-8 \pi^{2} U(\sin \theta / \lambda)^{2}\right]$.

CEstimated standard deviations are given in parelitheses for the least significant digits. 
Table B.3. Anisotropic Thermal Parametersa $\left(A^{2}, \times 10^{3}\right)$ for the Anion in $\left(\mathrm{NHC}_{5} \mathrm{H}_{5}\right) \mathrm{SbI}_{4}$

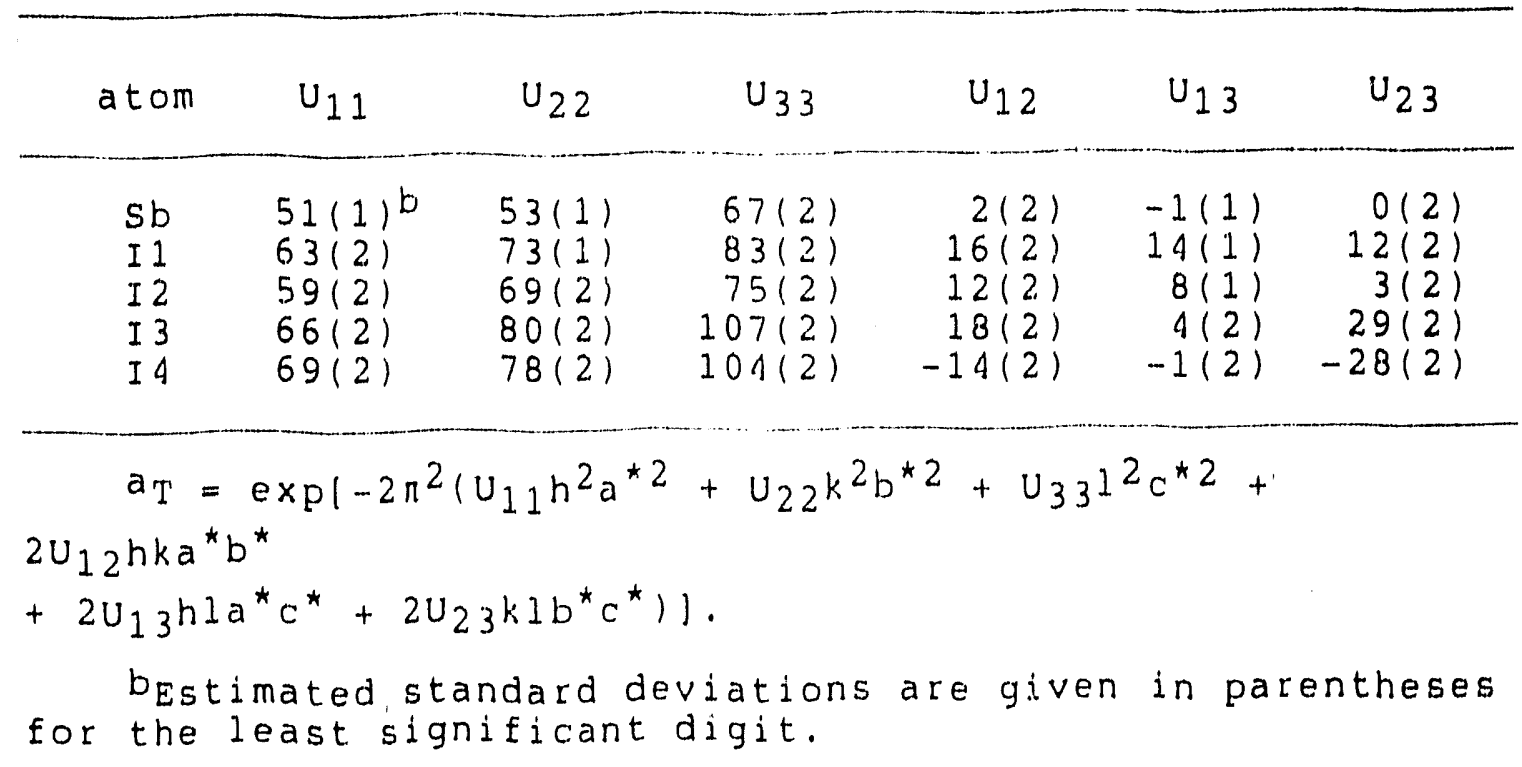


Table B.4. Selected Distances and Angles for $\left(\mathrm{C}_{5} \mathrm{H}_{5} \mathrm{NH}\right)_{\mathrm{SbI}}$

Distances $(\hat{A})$

St) $-I 1$

$S b-12$

$\mathrm{Sb}-\mathrm{I}^{3}$

I $2-\mathrm{N} 1$

$$
\begin{aligned}
& 3.021(4)^{\star} \\
& 3.037(3) \\
& 2.809(4) \\
& 3.807(31)
\end{aligned}
$$

$\begin{array}{ll}S b-I 1 a & 3.257(3) \\ S b-12 b & 3.340(3) \\ S b-14 & 2.828(4)\end{array}$

Angles (deg)

$\begin{array}{llll}\text { I } 1-S b-I 1 a & 91.80(9) & I 1-S b-I 2 b & 85.31(9) \\ \text { I1-Sb-I3 } & 87.94(10) & I 1-S b-I 4 & 92.96(10) \\ \text { I2-Sb-IIa } & 86.52(9) & I 2-S b-I 2 b & 92.05(8) \\ \text { I2-Sb-I3 } & 94.60(10) & I 2-S b-I A & 88.56(10) \\ \text { I3-Sb-I1a } & 89.60(10) & I 3-S b-I 4 & 93.99(10) \\ I 4-S b-I 2 b & 90.06(10) & I 1 a-S b-I 2 b & 86.92(8) \\ \text { NI-HI-I2 } & 153.07 & & \end{array}$

Subscripts refer to equivalent positions relative to the reference molecule.
(a)
$x, 1 / 2-y_{1} z-1 / 2$
(b)
$x_{1} 1 / 2-y_{1} 1 / 2-z$

*Estimated standard deviations are given in parentheses for the least significant digits. 
Table B.5. Positionala $\left(\times 10^{4}\right)$ and Isotropic Thermal $\left(A^{2}\right.$, $\times 1.0^{3}$ ) Parameters for the Hydrogen Atoms in $\left(\mathrm{C}_{5} \mathrm{H}_{5} \mathrm{NH}\right) \mathrm{SbI}_{4}$

\begin{tabular}{lrlll}
\hline atom & \multicolumn{1}{c}{$y$} & $z$ & $u^{b}$ \\
\hline H1 & 993 & 5015 & -7562 & 63.3 \\
H2 & 1843 & 5411 & -8618 & 63.3 \\
H3 & 2060 & 7175 & -8611 & 63.3 \\
H4 & 1429 & 8391 & -7676 & 63.3 \\
H6 & 577 & 7907 & -6630 & 63.3 \\
& 560 & 6180 & -6526 & 63.3 \\
\hline
\end{tabular}

ahydrogen atoms were not refined.

$b_{T}=\exp \left[-8 \pi^{2} U(\sin \theta / \lambda)^{2}\right)$. 


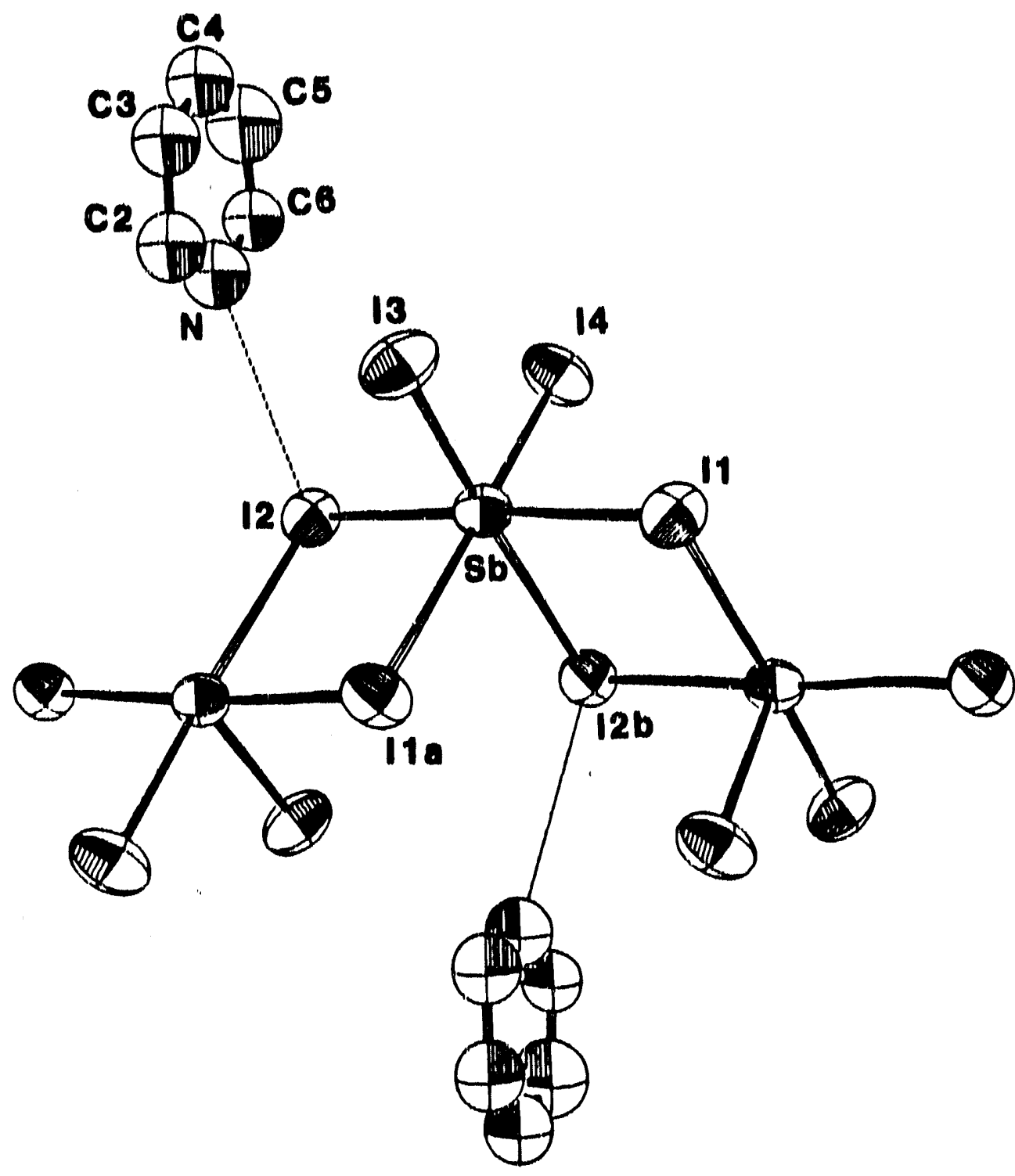

Figure B.1. View showing the immediate envitonment around the antimony atom in the structure of $\left(\mathrm{NHC}_{5} \mathrm{H}_{5}\right.$ ) Strig. Tho bridging iodines are it and I 2 


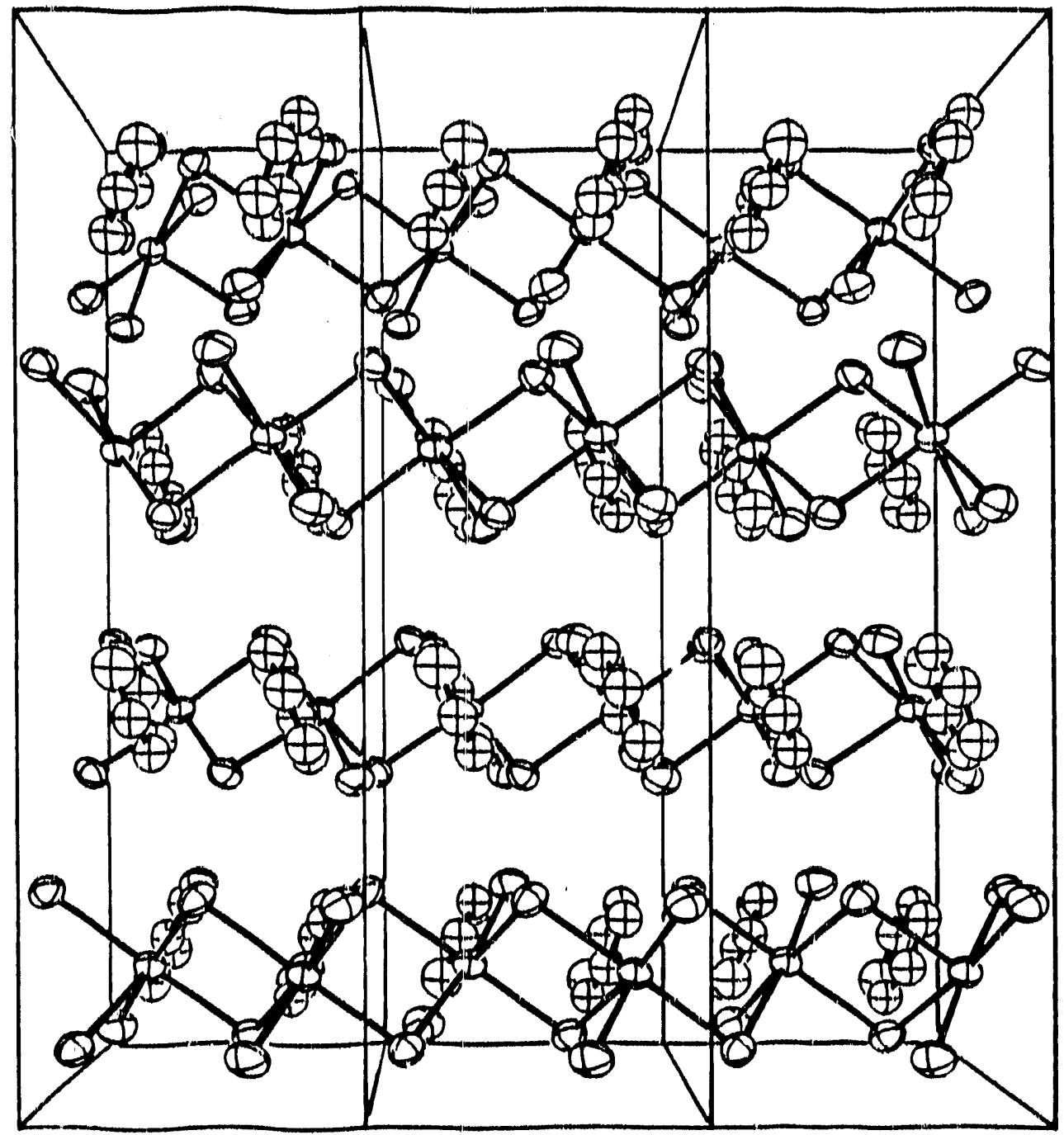

Figure B.2. Unit cell view of $\left(\mathrm{NHC}_{5} \mathrm{H}_{5}\right) \mathrm{SbI}$ a showing the infinite chain in the z-direction. In the figure, $c$ is horizontal, a is vertical and b is perpendicular to the plane of the paper 
.142

$3.037(3)$ A, respectively, while the longer bridging distances are $3.257(3)$ and $3.340(3)$ A. All are much shorter. than the sum of the van der wals radil, $1.35 \AA$.

Examination of the differences in bond length between the long and short bridge bonds in the three antimony-halogen compounds that form infinite chalns shows the following: chloro, $\Delta=0.496 ;$ bromo, $\Delta=0.408 ;$ and iodo, $\Delta=0.269 \mathrm{~A}$. Thus, there appears to be a clear trend toward less asymmetry in the bridge as the size of the halogen increases.

Because antimony halides often form as mixed-valence compounds, and it is difficult to distinguish pyridine from pyridinium by $x-r a y$ diffraction, one might speculate as to whether the antimony exists solely in the trivalent state or in a mixture of the tri-and pentavalent states. Using the approximation to the cation valence state given by Zachariasen 54 , and taking the $\mathrm{Sb}-\mathrm{I}$ bond distance of $2.67 \AA$ in $\mathrm{SbI}_{3}$ as the distance corresponding to a unit bond strength distance, the charge on the antimony is calculated to be 2.94, implying that the antimony is in the trivalent state. Also, in charge-transfer complexes, where the metal ion exists in a mixture of tri-and pentavalent states, the crystals appear jet black under reflected light. However, the crystal under examination appears orange under reflected light, which further suggests that the antimony is not in a mixed-valent state. Finaliy, the fact that the reaction 
143

mixture was am aciditic medium suggests; that the rimg is the

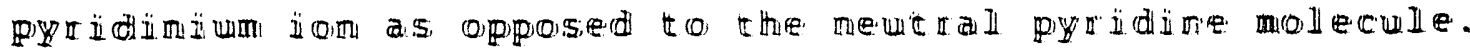

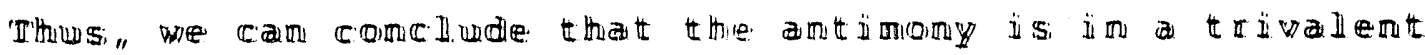
state:

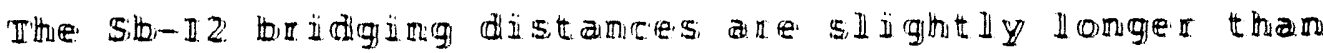

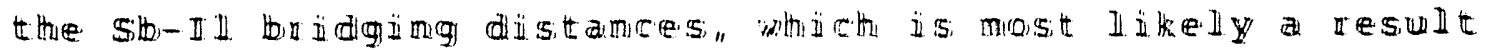

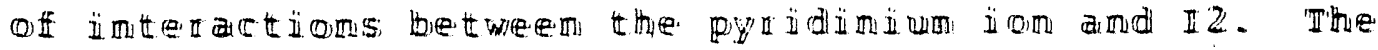

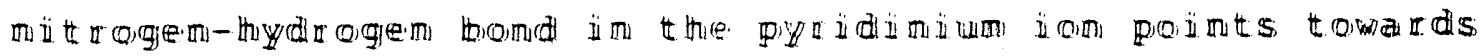

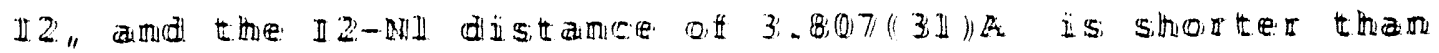

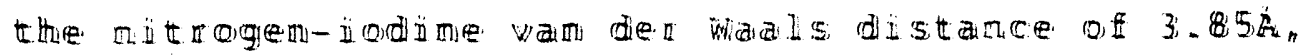
suggesting that there is thydrogem bomding occuratimg between the I 2 and the pyidimium rimg -

It is interestimg to mote that Zacthatiasen"s method

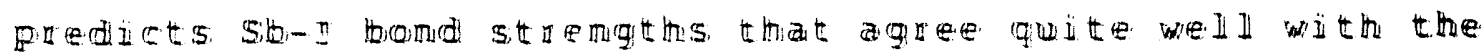

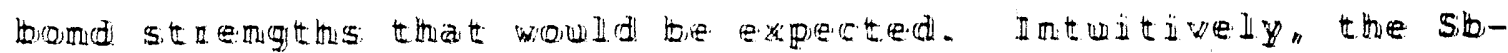

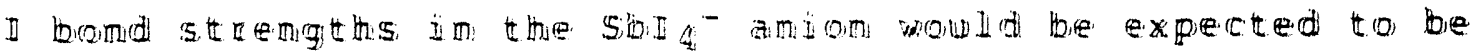

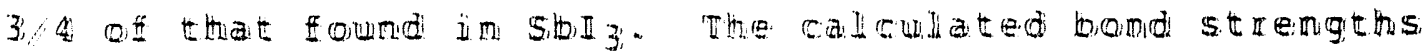

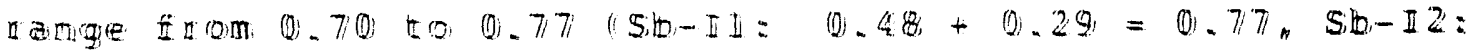
$0.46+0.24=0.70,50-13: 0.75,5,0-14=0.721$. Bucause 0.

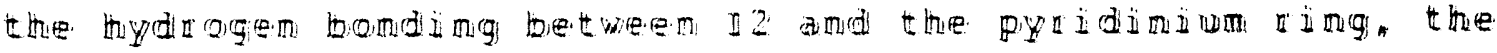
sit-I 2 bond would the experted to be the weakest "and the callcullation indicates that this is inderd the case.

Examidatiom of the lisotropte temperature factors of the

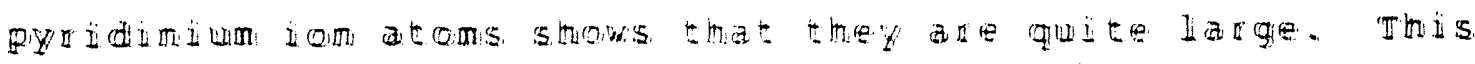
is comsistemt with the otsented tal off im imemsity of 
data thaving $\sin \theta / \lambda>0.5$. since thermal vibration effects are more promounced at large $\sin \theta / \lambda$ values.

$$
\text { Structure Determination of } \mathrm{PO}_{4} \mathrm{C}_{2} 4^{\mathrm{H}_{2}}
$$

\section{Experimental}

Crystal Data $\mathrm{PO}_{4} \mathrm{C}_{24} \mathrm{H}_{25}, M=408.43$, monoclinic, a $=$ $16.189(5), b=14.186(2), C=19.135(8) A, B=114.97(3)^{\circ}, \mathrm{V}$

$=4138.7 A^{3}$. space group P2lla from systematic absences

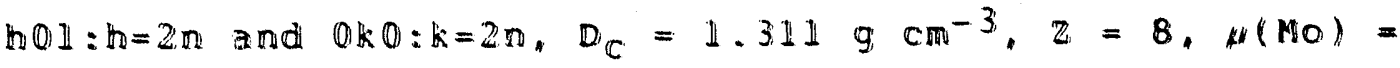
$1.5 .43 \mathrm{~cm}^{-1}$.

\section{Crystallographic studies crystals of this compound were} prepared in Dr. Verkade"s group "Department of Chemistry. Iowa state Universityh. For data collection, a crystal of approximate dimersions $0.1 \times 0.1 \times 0.1$ mo was mounted in a 0.2 mum diameter thin-waled capillary tube, sealed with wax, and attacthed to a standard goniometer thead. The crystal was aligned on a four-circle DATEX $x$-ray diffractometer. Four prelliminary w-oscillation photographs were taken at various \$. settings. The approximate positions of 15 reflections were sellected from the photographs and used as input to an autcratic indexing program 5.1 . The resulting reduced cell and reduced cell scalars indicated a primitive monoclinic lattice. The predicted layer spacings for this cell were olbserved" within experimental eror, on three axial (woscillation photograpins. 
Intensity data were collected at room temperature $\left(20^{\circ} \mathrm{C}\right)$ using Mo $K_{\alpha}(\lambda=0.70169$ A $)$ radiation on the above mentioned diffractometer equipped with a graphite monochromating crystal and interfaced to a VAX 370 computer in a real-time mode. A total of 4768 intensities were measured, corresponding to reflections in octants $h k l$ and $\bar{h} k l$, using an w-step scan technique with a scan half-width of 0.5 degree. The data were collected to a maximum $\sin \theta / \lambda$ of 0.6 . As a check on the stability of the crystal, the intensities of seven standard reflections were measured every 50 reflections during data collection. No significant decay was abserved.

Accurate unit cell parameters were obtained by carefully centering ten independent high-angle reflections on the diffractometer noted above and inputing the $\pm 2 \theta$ values of these reflections into a least-squares fitting program. Intensity data were corrected for Lorentz-polarization effects, but not for absorption due to the low value of $\mu$. of the 4758 refiections measured, the 2808 that had $I \geq$ 30 (I) were considered to be observed. The symmetry equivalent reflections were averaged $\left(R_{a v}=0.028\right)$, yielding 2649 reflections which were used in the refinement of the structure. 
Solution and Refinement

The positions of the non-hydrogen atoms were determined as follows: Patterson superpositions were carried out using (i) the arglide phosphorus-phosphorus Harker vector, followed by (ii) the 21 phosphorus-phosphorus Harker vector. The resulting superposition map showed a quartet of large peaks, which were taken to be phosphorus atoms. Further analysis showed a second quartet of peaks, also quite large in the original patterson, which had the same relationships amongst themselves as did the initial quartet of peaks. This implied the preserice of two parallelograms of phosphorus atoms, having the vectors that were chosen for the superpositions in common. From these two quartets, the positions of two non-symmetry-related phosphorus atoms were determined. The positions of the remaining non-hydrogen atoms were determined from successive structure factor 52 and electron density map calculations 53 .

The initial positional and isotropic thermal parameters were refined using a block-matrix least-squares procedure. The positional and anisotropic thermal parameters were refined to their final values using a full-matrix leastsquares procedure, minimizing the function $\sum w\left(\left|F_{0}\right|-\left|F_{C}\right|\right)^{2}$ to a conventional residual index of $R=\sum\left(\left|\left(\left|F_{O}\right|-\left|F_{C}\right|\right)\right|\right) / \Sigma\left|F_{O}\right|$ $=0.047$ and a weighted residual index of $R_{W}=\mid \Sigma W\left(\left|F_{0}\right|-\right.$ $\left.\left|F_{C}\right|\right)^{2} /\left[w\left|F_{0}\right|^{2}\right]^{1 / 2}=0.047$, where $w=1 / 0^{2}\left(\left|F_{0}\right|\right)$. The positions of approximately two-thirds of the hydrogens were 


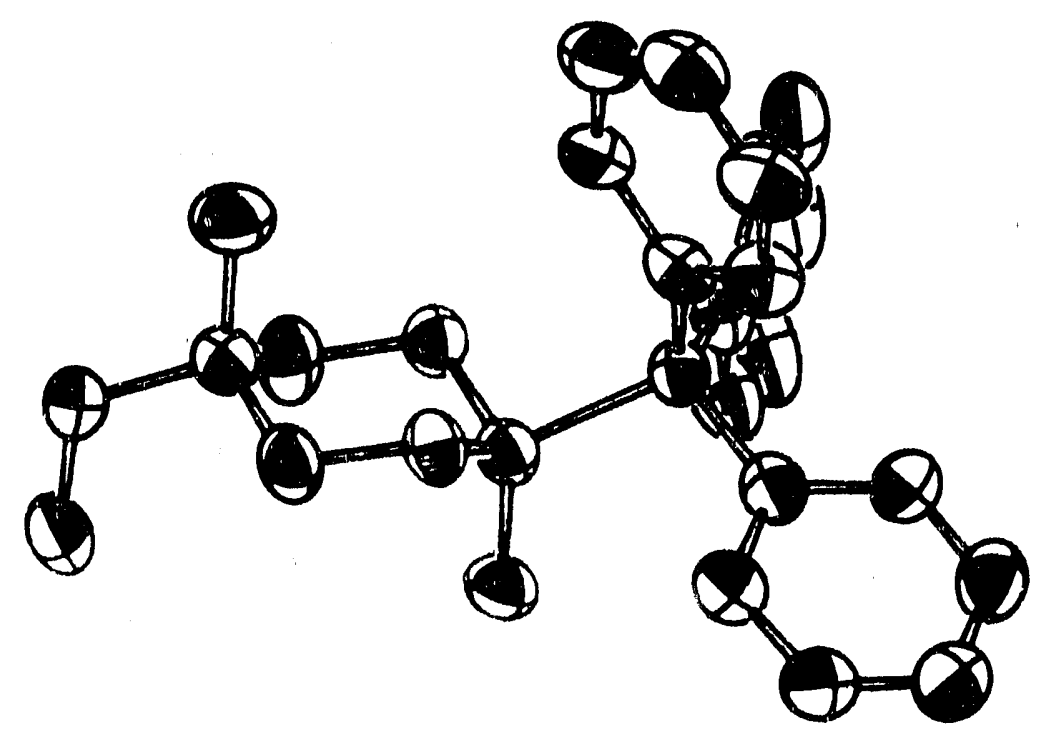

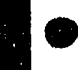

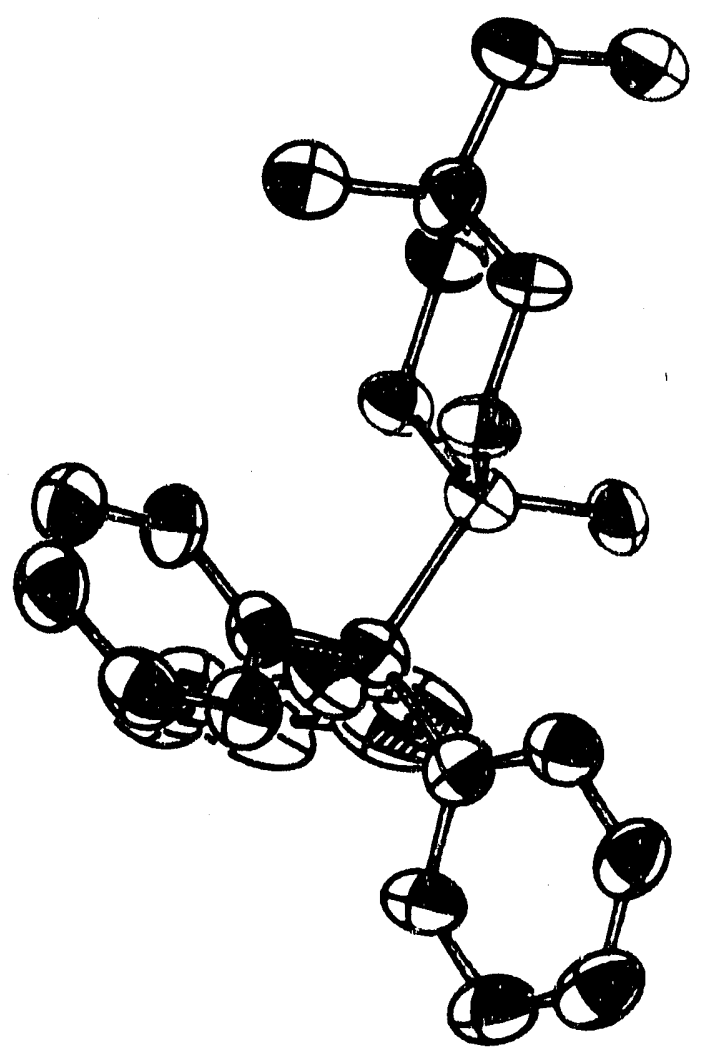

Figure B.3. ORTEP drawing for $\mathrm{PO}_{4} \mathrm{C}_{2} \mathrm{H}_{25}$ 
Table B.6. Positionala $\left(\times 10^{4}\right)$ and Averaged Isotropic Thermal $\left(A^{2}, \times 10^{3}\right)$ Parameters for $\mathrm{PO}_{4} \mathrm{C}_{24} \mathrm{H}_{25}$

\begin{tabular}{|c|c|c|c|c|}
\hline atom & $x$ & $Y$ & $z$ & $u_{a v^{b}}^{b}$ \\
\hline 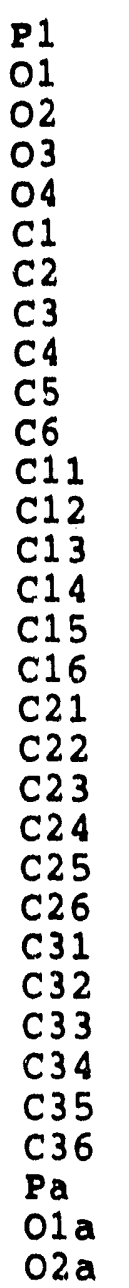 & $\begin{array}{r}9142(1)^{C} \\
9508(3) \\
8674(3) \\
8399(3) \\
6707(3) \\
9942(4) \\
7857(4) \\
7588(4) \\
7183(4) \\
6415(4) \\
6806(5) \\
9626(4) \\
10140(5) \\
9845(6) \\
9048(5) \\
8526(5) \\
8805(4) \\
10830(4) \\
10849(5) \\
11656(5) \\
12443(5) \\
12411(5) \\
11622(5) \\
10041(4) \\
9868(4) \\
9947(5) \\
10173(6) \\
10398(6) \\
10311(5) \\
9621(1) \\
10497(3) \\
9384(3)\end{array}$ & $\begin{array}{r}2327(1) \\
2711(3) \\
1336(3) \\
2953(3) \\
2536(4) \\
2157(5) \\
1370(4) \\
3015(4) \\
2041(5) \\
2159(5) \\
1644(5) \\
1396(4) \\
596(5) \\
-75(5) \\
29(5) \\
813(6) \\
1487(5) \\
1839(5) \\
1175(5) \\
806(5) \\
1149(5) \\
1817(6) \\
2162(5) \\
3149(5) \\
3293(6) \\
4201(7) \\
4948(6) \\
4811(6) \\
3907(5) \\
2326(2) \\
2680(4) \\
1346(3)\end{array}$ & $\begin{array}{r}9657(1) \\
9140(2) \\
9378(2) \\
9719(2) \\
7402(2) \\
10684(3) \\
8669(4) \\
8996(4) \\
8740(4) \\
7931(4) \\
9287(4) \\
11076(3) \\
11420(4) \\
11785(4) \\
11837(4) \\
11494(4) \\
11110(4) \\
10683(4) \\
10159(4) \\
10214(4) \\
10797(5) \\
11318(4) \\
11268(4) \\
11054(4) \\
11706(4) \\
12023(5) \\
11690(5) \\
11070(5) \\
10756(4) \\
5366(1) \\
5885(3) \\
5637(3)\end{array}$ & $\begin{array}{l}42 \\
52 \\
45 \\
48 \\
67 \\
40 \\
48 \\
49 \\
46 \\
55 \\
71 \\
41 \\
53 \\
66 \\
63 \\
62 \\
50 \\
43 \\
51 \\
55 \\
62 \\
57 \\
54 \\
45 \\
54 \\
65 \\
67 \\
69 \\
60 \\
46 \\
49 \\
51\end{array}$ \\
\hline
\end{tabular}

apositional parameters are given as fractions of the unit cell.

$$
\begin{aligned}
& b_{U_{a v}}=\left(U_{11}+U_{22}+U_{33}\right) / 3 . T=\exp l-2 \pi^{2}\left(U_{11} h^{2} a^{\star 2}+\right. \\
& U_{22} k^{2} b^{\star 2}+U_{33} l^{2} c^{\star 2}+2 U_{12} h k a^{\star} b^{\star}+2 U_{13} h l a^{\star} c^{\star}+
\end{aligned}
$$
$\left.\left.2 U_{23} k 1 b^{\star} c^{\star}\right)\right]$.

Estimated standard deviations are given in parentheses for the least significant digit. 
Table B.6. (Continued)

\begin{tabular}{|c|c|c|c|c|}
\hline atom & $x$ & $y$ & $z$ & $\mathrm{u}_{\mathrm{av}}$ \\
\hline 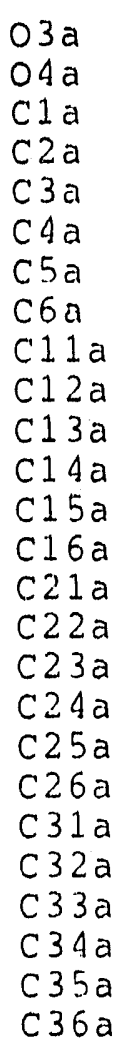 & $\begin{array}{r}8854(3) \\
9358(3) \\
9430(5) \\
9260(5) \\
8724(5) \\
8550(5) \\
8528(5) \\
7641(5) \\
9241(6) \\
8453(6) \\
8299(7) \\
8940(10) \\
9742(8) \\
9890(7) \\
10269(5) \\
10455(6) \\
11175(6) \\
11730(6) \\
11541(5) \\
10820(5) \\
8658(5) \\
8776(5) \\
8083(7) \\
7260(6) \\
7142(6) \\
7842(6)\end{array}$ & $\begin{array}{l}3002(3) \\
2542(4) \\
2156(5) \\
1395(5) \\
3061(5) \\
2088(6) \\
2198(7) \\
1718(6) \\
3155(6) \\
3417(6) \\
4357(8) \\
5037(8) \\
4779(8) \\
3851(7) \\
1783(5) \\
2005(6) \\
1624(7) \\
1005(7) \\
762(6) \\
1126(6) \\
1461(5) \\
630(6) \\
11(6) \\
203(7) \\
1023(6) \\
1665(6)\end{array}$ & $\begin{array}{l}5322(3) \\
7635(3) \\
4342(4) \\
6358(4) \\
6042(4) \\
6296(5) \\
7093(4) \\
5743(5) \\
4004(5) \\
3381(5) \\
3120(5) \\
3472(7) \\
4054(6) \\
4315(5) \\
4300(5) \\
3672(4) \\
3600(5) \\
4150(6) \\
4765(5) \\
4844(4) \\
3931(4) \\
3601(5) \\
3240(5) \\
3204(5) \\
3553(5) \\
3921(4)\end{array}$ & $\begin{array}{l}49 \\
72 \\
39 \\
51 \\
52 \\
50 \\
72 \\
72 \\
50 \\
62 \\
87 \\
106 \\
93 \\
72 \\
42 \\
59 \\
67 \\
69 \\
58 \\
51 \\
42 \\
53 \\
62 \\
60 \\
58 \\
48\end{array}$ \\
\hline
\end{tabular}


Table B.7. Andsotroplo Thermal parametersa $\left(A^{2}, \times 10^{3}\right)$ for the Non-Hydrogen Atoms in $\mathrm{PO}_{4} \mathrm{C}_{2} 4_{4} \mathrm{H}_{25}$

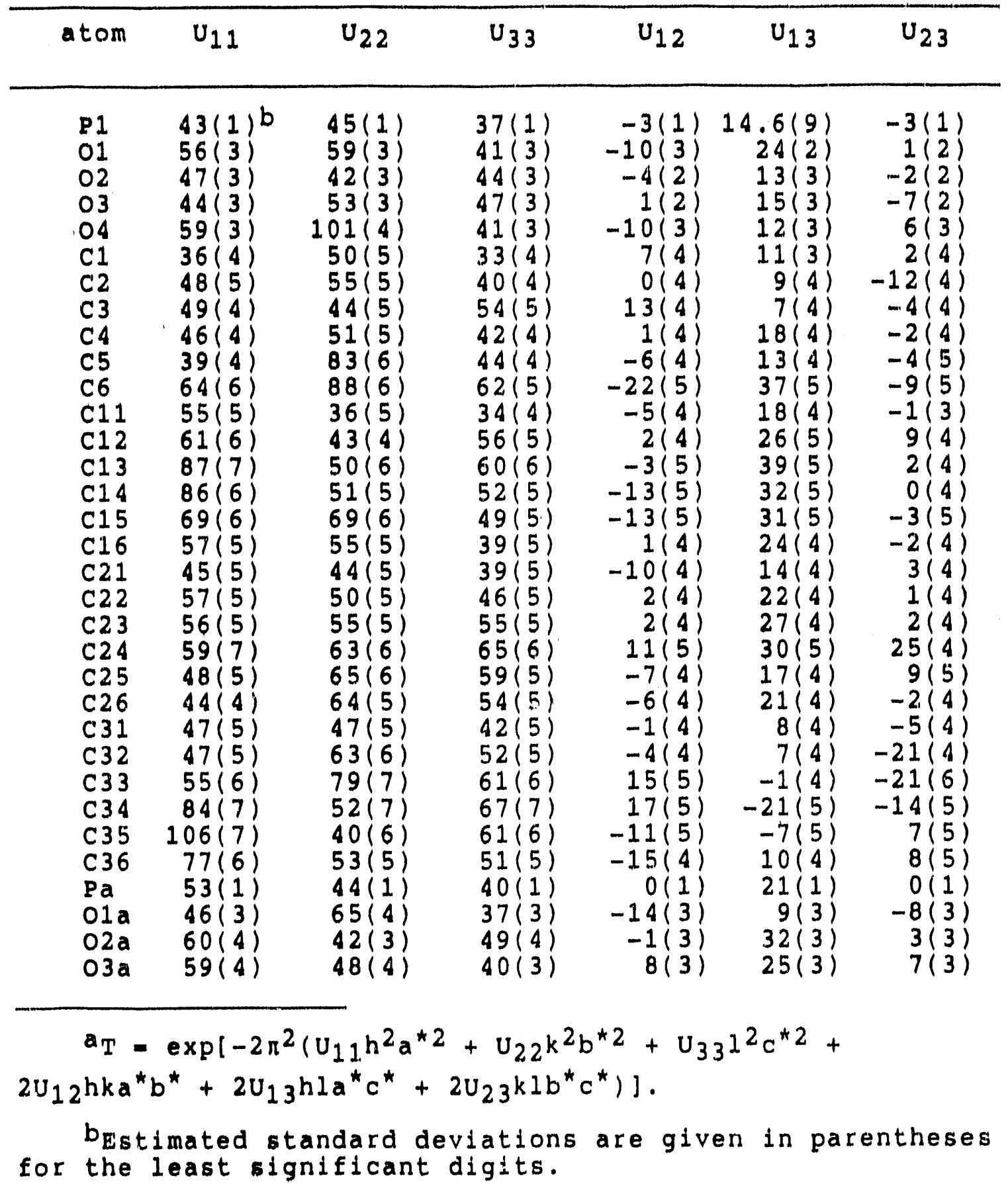


Table B.7. (Continued)

\begin{tabular}{rrrrrrr}
\hline atom & $U_{1} 1$ & $U_{22}$ & $U_{33}$ & $U_{12}$ & $U_{13}$ & $U_{23}$ \\
\hline O4a & $70(4)$ & $100(4)$ & $47(4)$ & $-13(4)$ & $28(3)$ & $-10(4)$ \\
C1a & $51(5)$ & $32(5)$ & $35(4)$ & $8(4)$ & $16(4)$ & $6(4)$ \\
C2a & $63(6)$ & $44(6)$ & $45(5)$ & $-1(5)$ & $36(5)$ & $2(4)$ \\
C3a & $64(6)$ & $43(5)$ & $48(5)$ & $1(5)$ & $29(5)$ & $-3(4)$ \\
C4a & $40(5)$ & $51(6)$ & $60(6)$ & $-13(5)$ & $20(5)$ & $-5(5)$ \\
C5a & $70(6)$ & $97(8)$ & $48(5)$ & $-12(6)$ & $32(5)$ & $-5(6)$ \\
C6a & $68(6)$ & $86(7)$ & $62(6)$ & $2(5)$ & $31(5)$ & $-8(5)$ \\
C11a & $76(7)$ & $31(5)$ & $43(6)$ & $1(5)$ & $25(5)$ & $-7(5)$ \\
C12a & $87(7)$ & $45(7)$ & $55(6)$ & $18(6)$ & $35(6)$ & $17(5)$ \\
C13a $118(9)$ & $80(7)$ & $62(7)$ & $38(7)$ & $49(7)$ & $19(6)$ \\
C14a $198(14)$ & $44(8)$ & $76(9)$ & $17(9)$ & $78(9)$ & $8(7)$ \\
C15a $164(12)$ & $53(8)$ & $64(7)$ & $-27(7)$ & $61(8)$ & $-13(6)$ \\
C16a $114(9)$ & $53(7)$ & $50(6)$ & $-17(7)$ & $46(6)$ & $-12(6)$ \\
C21a & $14(5)$ & $32(5)$ & $51(6)$ & $-10(4)$ & $20(5)$ & $-16(4)$ \\
C22a & $68(7)$ & $60(6)$ & $49(6)$ & $-6(5)$ & $35(5)$ & $-3(5)$ \\
C23a & $62(7)$ & $69(7)$ & $69(7)$ & $-7(6)$ & $41(6)$ & $-1(6)$ \\
C24a & $59(7)$ & $58(7)$ & $89(7)$ & $-9(6)$ & $42(6)$ & $-21(6)$ \\
C25a & $45(6)$ & $57(6)$ & $72(7)$ & $-8(5)$ & $24(5)$ & $-18(5)$ \\
C26a & $59(6)$ & $47(6)$ & $48(6)$ & $-12(5)$ & $25(5)$ & $-8(5)$ \\
C31a & $44(6)$ & $41(6)$ & $41(5)$ & $-11(5)$ & $12(4)$ & $-1(5)$ \\
C32a & $59(6)$ & $44(5)$ & $57(6)$ & $-16(5)$ & $22(5)$ & $-12(5)$ \\
C33a & $75(7)$ & $57(7)$ & $54(6)$ & $-9(6)$ & $26(6)$ & $-13(5)$ \\
C34a $345(6)$ & $64(7)$ & $61(6)$ & $-18(6)$ & $14(5)$ & $-10(5)$ \\
C35a & $49(7)$ & $66(6)$ & $59(6)$ & $9(5)$ & $18(5)$ & $2(5)$ \\
C36a & $42(6)$ & $48(6)$ & $52(5)$ & $-8(5)$ & $11(5)$ & $-6(5)$ \\
& & & & & & \\
\hline
\end{tabular}


Table B.8. Pooltionala $\left(\times 10^{4}\right)$ and Thermal $\left(A^{2}, \times 10^{3}\right)$

parameter for Hydrogen Atoms in $\mathrm{PO}_{4} \mathrm{C}_{2}{ }_{4} \mathrm{H}_{25}$

\begin{tabular}{|c|c|c|c|c|}
\hline atom & $x$ & $y$ & $\mathbf{z}$ & $u^{b}$ \\
\hline $\begin{array}{l}\text { H1 } \\
H 2 \\
H 3 \\
H 4 \\
H 5 \\
H 6 \\
H 71 \\
H 81 \\
H 91 \\
H 72 \\
H 82 \\
H 92 \\
H 10 \\
H 12 \\
H 13 \\
H 14 \\
H 15 \\
H 16 \\
H 22 \\
H 23 \\
H 24 \\
H 25 \\
H 26 \\
H 32 \\
H 33 \\
H 34 \\
H 35 \\
H 36 \\
H 1 a \\
H 2 a \\
H 3 a \\
H 4 a \\
H 5 a \\
H 6 a \\
H 7 a 1 \\
H 8 a 1 \\
H 9 a 1 \\
H 7 a 2 \\
H 8 a 2\end{array}$ & $\begin{array}{l}7887 \\
7553 \\
7740 \\
7303 \\
5883 \\
6013 \\
6340 \\
7282 \\
6580 \\
7129 \\
6887 \\
6186 \\
6263 \\
10802 \\
10260 \\
8822 \\
7899 \\
8392 \\
10216 \\
11665 \\
13086 \\
13027 \\
11600 \\
9649 \\
9779 \\
10238 \\
10626 \\
10495 \\
9250 \\
9831 \\
8181 \\
9262 \\
8375 \\
8033 \\
7160 \\
7523 \\
77583 \\
7684 \\
7261\end{array}$ & 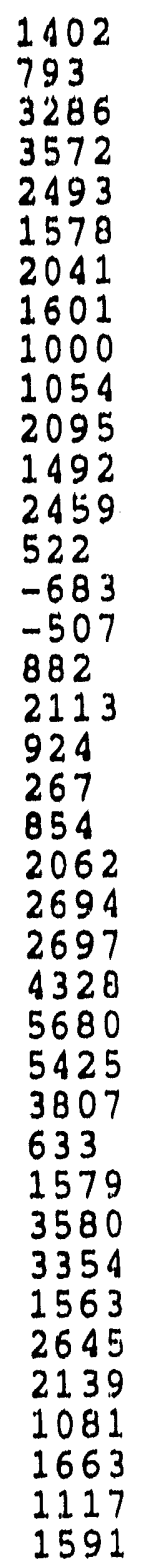 & $\begin{array}{l}8142 \\
8500 \\
8566 \\
9083 \\
8024 \\
7773 \\
9293 \\
9815 \\
9122 \\
9528 \\
9698 \\
9004 \\
6847 \\
11388 \\
12055 \\
112127 \\
11526 \\
10837 \\
9703 \\
9795 \\
10831 \\
11769 \\
11679 \\
11963 \\
12535 \\
11931 \\
10806 \\
10254 \\
6528 \\
6807 \\
65 \\
5902 \\
6465 \\
7251 \\
7039 \\
5722 \\
\end{array}$ & $\begin{array}{l}76.0 \\
76.0 \\
76.0 \\
76.0 \\
76.0 \\
76.0 \\
76.0 \\
76.0 \\
76.0 \\
76.0 \\
76.0 \\
76.0 \\
76.0 \\
76.0 \\
76.0 \\
76.0 \\
76.0 \\
76.0 \\
76.0 \\
76.0 \\
76.0 \\
76.0 \\
76.0 \\
76.0 \\
76.0 \\
76.0 \\
76.0 \\
76.0 \\
76.0 \\
76.0 \\
76.0 \\
76.0 \\
76.0 \\
76.0 \\
76.0 \\
76.0 \\
76.0 \\
76.0 \\
76.0\end{array}$ \\
\hline
\end{tabular}

a Hydrogen positions were calculated but not refined. $b_{T}=\exp \left[-8 \pi^{2} U(\sin \theta / \lambda)^{2}\right]$. 
Table B.8. (Continued)

\begin{tabular}{|c|c|c|c|c|}
\hline atom & $x$ & $y$ & $\%$ & $u^{b}$ \\
\hline $\begin{array}{l}H 9 a 2 \\
H 10 a \\
H 12 a \\
H 13 a \\
H 14 a \\
H 15 a \\
H 16 a \\
H 22 a \\
H 23 a \\
H 24 a \\
H 25 a \\
H 26 a \\
H 32 a \\
H 33 a \\
H 34 a \\
135 a \\
136 a\end{array}$ & $\begin{array}{l}7321 \\
9391 \\
7921 \\
7665 \\
8827 \\
10261 \\
10543 \\
10011 \\
11323 \\
12321 \\
11985 \\
10662 \\
9139 \\
8191 \\
6692 \\
6172 \\
7731\end{array}$ & $\begin{array}{l}2175 \\
2333 \\
2863 \\
1561 \\
5793 \\
5323 \\
3658 \\
2516 \\
1839 \\
709 \\
212 \\
910 \\
171 \\
-656 \\
-282 \\
1202 \\
2328\end{array}$ & $\begin{array}{l}5318 \\
8145 \\
3091 \\
2526 \\
3266 \\
1338 \\
4808 \\
3228 \\
3098 \\
1072 \\
5199 \\
5346 \\
3598 \\
2968 \\
2932 \\
3554 \\
1202\end{array}$ & $\begin{array}{l}76.0 \\
76.0 \\
76.0 \\
76.0 \\
76.0 \\
76.0 \\
76.0 \\
76.0 \\
76.0 \\
76.0 \\
76.0 \\
76.0 \\
76.0 \\
76.0 \\
76.0 \\
76.0 \\
76.0\end{array}$ \\
\hline
\end{tabular}


determinod from a difforence aloction density map and tha rost of the hydrogen postelons wate calculated. None of the hydiogen positiona were refined. The final postelonal and averaged dootiopic thermed patameters for the non-hydrogen atoms are glven in lable B.6, and the andsotrople thermal palameters for these atoms ate glven in table B.7. Table B. B containg the calculated positions and lootropie thermal parametes for the hydrogen atoms.

$$
\text { Stiuctule Determination of } \mathrm{PO}_{2} \mathrm{C}_{6} \mathrm{H}_{11}
$$

Expetimental

Clystal Data $\mathrm{PO}_{2} \mathrm{C}_{6} \mathrm{H}_{1}, \mathrm{M}$ - 119.13 , orthorhombic, a $10.2107(12), b=10.5511(23), c=6.1639(6) A, V=697.51$ $A^{3}$, spacte group P2 $122_{1}$ from systematic absences hoo:hw2n, $0 k 0: k=2 n$ and $001: 1=2 n, D_{C}=1.391 \mathrm{~g} \mathrm{Cm}^{-3}, 2=1, \mu(\mathrm{MO})=$ $3.077 \mathrm{~cm}^{-1}$.

Crystadlograplide studies crystals of this compound were prepared in Dr. Verkade's group (Department of Chemistry, Jowa state University). For data collection, a cylindrical crystal of approximate dimensions $0.35 \mathrm{~mm}$ in length and 0.15 mon in diameter was mounted in a 0.2 mm diameter thin-walled capillary tube, sealed with wax, and attached to a standard goniometer head. The cryetal was aligned on a four-circle SYNTEX $x$-ray diferactometer. A preliminary rotation photograph was taken. The approximate positions of 1.2 
Leflectiong wate belocted fiom the photograph, refined and uesed as input to an automatio indexing program42. The liesulting reduced celit and reducod cell sealars indicated a primitive orthorhomble lattice. The predicted layer spacings for this cold wate observed, within experimental

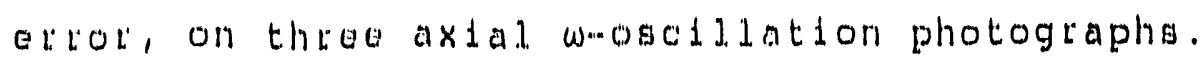

Intonsliy data were collected at room temperature $\left(20^{\circ} \mathrm{C}\right)$ uaing Mo $K_{\alpha}(\lambda m 0.70169$ A $)$ lidiation on the above mentioned diffiactometer equipped with a graphite monochromating cryatal and interfaced to a NOVA computer in a real-time mode. A total of $186 \%$ intengltios were measured, cotiesponding to reflections in octants hkl and Fkl, using an w-step scan technicule with a scan half-width of 0.5 dogree. The data were collected to an maximum $\sin \theta / \lambda$ of 0.6. As a check on the stablitty of the crystal, the intensity of one standard reflection was measured every 75 reflections during data collection. No stgniflcant decay was observed.

Acculate unt cell parameters were obtalned by carefulily centering fifteen independent high-angle reflections on the diffractometer noted above and inputing the $\pm 2 \theta$ values of these reflections into a least-squares fitting program. Intensity data were corrected for Lorentz-polarization effects, but not for absorption due to the low value of $\mu$. of the 1867 reflections measured, the 918 that had I $\geq 30$ (I) 

Tabile B.9. Powitional $\left(x 10^{4}\right)$ and Averaged Isotropic Thermal $\left(\mathrm{A}^{2}\right.$, w $\left.10^{3}\right)$ Parameters for $\mathrm{PO}_{2} \mathrm{C}_{6} \mathrm{H}_{11}$

\begin{tabular}{|c|c|c|c|c|}
\hline atom & $x$ & $y$ & $a$ & $u_{a v}$ \\
\hline $\begin{array}{l}\text { P1 } \\
01 \\
02 \\
c 1 \\
c 2 \\
c 3 \\
c 4 \\
c 5 \\
c 6\end{array}$ & $\begin{array}{r}5755(3) c \\
5687(10) \\
5555(19) \\
617(16) \\
4116(12) \\
4607(19) \\
5091(16) \\
3338(12) \\
4088(16)\end{array}$ & $\begin{array}{r}3377(13) \\
3054(11) \\
2227(16) \\
4655(112) \\
3795(111) \\
2491(12) \\
4745(12) \\
3948(116) \\
3752(11)\end{array}$ & $\begin{array}{l}7840(14) \\
9516(16) \\
6243(14) \\
6206(24) \\
8546(16) \\
4545(22) \\
4469(20) \\
6569(20) \\
4669(17)\end{array}$ & $\begin{array}{l}42 \\
88 \\
57 \\
67 \\
52 \\
92 \\
81 \\
79 \\
55\end{array}$ \\
\hline
\end{tabular}

apositional parameters are given as fractions of the unit cell.

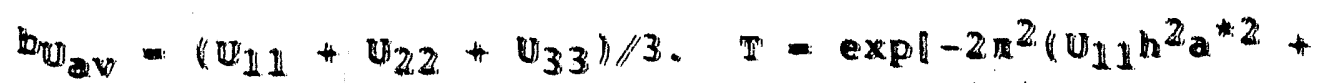
$U_{22} k^{2} b^{m}+U_{33} 1^{2} c^{m 2}+2 U_{12} 2_{k a}^{*} b^{*}+2 U_{13} h 1 a^{*} c^{*}$ $\left.202310 * c^{*}\right) 1$.

Castimated stindard deviations are given in parentheses for the least sighificant digit. 


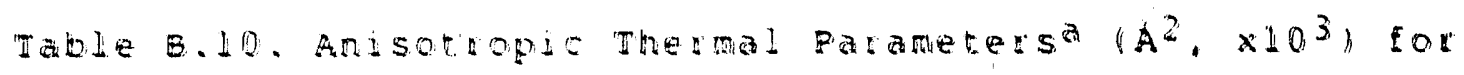
the Non-Hydrogen Atoms in $\mathrm{PO}_{2} \mathrm{C}_{5} \mathrm{H}_{1}$

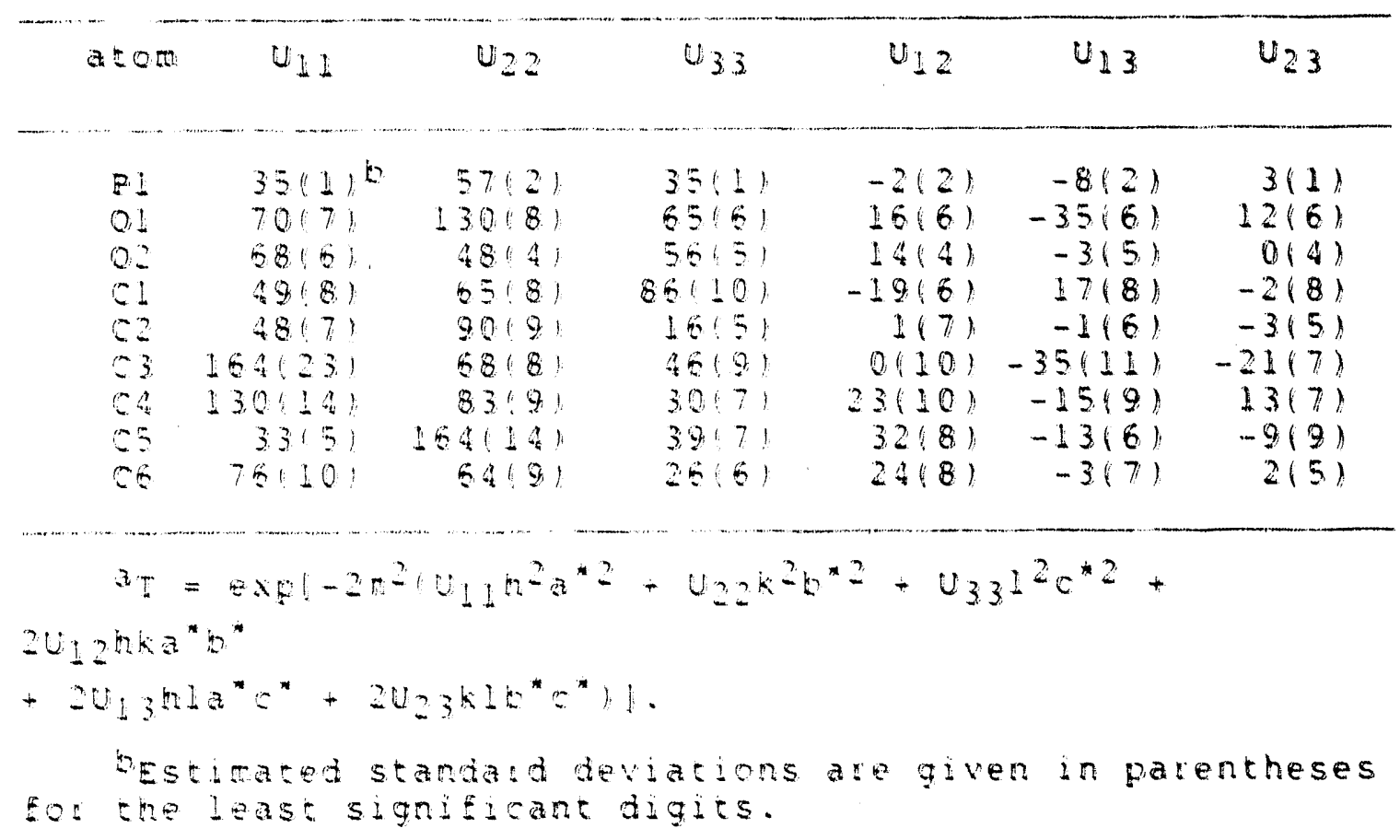


Table G.11. Fositionala $\left(\times 10^{4}\right)$ and Tlermal $\left(A^{2}, \times 10^{3}\right)$ Parameters for Hydrogen Atoms in $\mathrm{PO}_{2} \mathrm{C}_{6} \mathrm{H}_{11}$

\begin{tabular}{ccccc} 
atom & $x$ & $y$ & $z$ & $u^{b}$ \\
$H 1$ & 7127 & 486 & 5469 & 38.0 \\
$H 2$ & 6201 & 5537 & 7018 & 38.0 \\
$H 3$ & 4101 & 4671 & 9411 & 38.0 \\
$H 4$ & 3679 & 3046 & 9492 & 38.0 \\
$H 5$ & 3882 & 1783 & 4538 & 38.0 \\
$H 6$ & 5202 & 2386 & 3067 & 38.0 \\
$H 7$ & 5609 & 4632 & 2974 & 38.0 \\
$H 8$ & 4669 & 5660 & 4542 & 38.0 \\
$H 9$ & 2914 & 4891 & 6542 & 38.0 \\
$H 10$ & 2533 & 3255 & 6581 & 38.0 \\
$H 11$ & 3236 & 3943 & 3392 & 38.0 \\
\hline
\end{tabular}

${ }^{a} H y d r o g e n$ positions were calculated but not refined. $b_{T}=\exp \left(-8 \pi-u\left(\sin \theta \lambda^{2}\right)\right.$. 
were considered to be observed. The symmetry equivalent reflections were averaged $\left(R_{a v}=0.099\right)$, yielding 542 reflections which were used in the refinement of the structure.

\section{Solution and Refinement.}

The position of the phosphorus atom was determined by examination of a patterson map. The positions of the remaining non-hydrogen atoms were determined from successive structure factor 43 and electron density map calculations 51 . The initial positional and isotropic thermal parameters were refined using a block-matrix least-squares procedure. The positional and anisotropic thermal parameters were refined to theil final values using a full-matrix least-squares procedure, minimiaing the function $\left[w\left(\left|F_{0}\right|-\left|F_{C}\right|\right)^{2}\right.$ to a conventional tesidual index of $R=\Sigma\left(\left|\left(\left|F_{O}\right|-\left|F_{C}\right|\right)\right|\right) / \Sigma\left|F_{O}\right|=$ 0.088 and a weighted residual index of $R_{W}=|\Sigma W|\left|F_{0}\right|-$ $\left|F_{C}\right|^{2}\left[W\left|F_{O}\right|^{2}\right]^{2}=0.095$, where $w=1 \sigma^{2}\left(\left|F_{0}\right|\right)$. The positions of the hydrogen atoms were calculated and not refined. The final positional and averaged isotropic thermal parameters for the non-hydrogen atoms are given in Table B.9, and the anisotropic thermal parameters for these atoms ale given in Table B.10. Table B.11 contains the calculated positions and isotropic thermal parameters for the hydrogen atoms. 


\section{Experimental}

Crystal Data $\left(\mathrm{NHC}_{5} \mathrm{H}_{5}\right)_{2} \mathrm{I}_{10}, \mathrm{M}=1429.26$, orthorhombic, a $=17.3305(37), b=17.6146(26), c=4.5336(7) \AA, V=$ $1383.996 \mathrm{~A}^{3}$, space group Pm2 $\mathrm{b}^{\mathrm{b}}$, systematic absences $h k 0: k=2 \mathrm{n}$ and $0 k 0: k=2 n, D_{C}=3.429 \mathrm{~g} \mathrm{~cm}^{-3}, Z=2, \mu\left(M_{O}\right)=110.8 \mathrm{~cm}^{-1}$. Crystallographic studies for data collection, a crystal of approximate dimensions $0.35 \times 0.15 \times 0.15 \mathrm{~mm}$ was mounted in a $0.2 \mathrm{~mm}$ diameter thin-walled capillary tube, sealed with wax, and attached to a standard goniometer head. The crystal was aligned on a four-circle SYNTEX X-ray diffractometer. A preliminary rotation photograph was taken. The approximate positions of 13 reflections were selected from the photograph, refined and used as input to an automatic indexing program 42 . The resulting reduced cell and reduced cell scalars indicated a primitive orthorhombic lattice. The predicted layer spacings for this cell were observed, within experimental error, on three axial woscillation photographs.

Intensity data were collected at room temperature $\left(20^{\circ} \mathrm{C}\right)$ using MO $K_{\alpha}(\lambda=0.70169 \mathrm{~A})$ radiation on the above mentioned diffractometer equipped with a graphite monochromating crystal and interfaced to a NOVA computer in a real-time mode. A total of 1827 intensities were measured, corresponding to reflections in the hkl octant, using an w- 
step scan technique with a scan half-width of 0.5 degree. The data were collected to an maximum $\sin \theta / \lambda$ of 0.6 . As a check on the stability of the crystal, the intensity of one standard reflection was measured every 75 reflections during data collection. No significant decay was observed.

Accurate unit cell parameters were obtained by carefully centering fifteen independent high-angle reflections on the diffractometer noted above and inputing the $\pm 2 \theta$ values of these reflections into a least-squares fitting program. Intensity data were corrected for absorption $\left(\mathrm{T}_{\mathrm{min}} / \mathrm{T}_{\max }=\right.$ $0.180 / 0.219)$, as well as for Lorentz-polarization effects. of the 1827 reflections measured, the 555 that had I $\geq 3 \sigma(I)$ were considered to be observed.

\section{Solution and Refinement}

The correct space group and the locations of two iodines were determined as described in Chapter 5. The positions of the remaining non-hydrogen atoms were determined from successive structure factor 43 and electron density map calculations 51 . The initial positional and isotropic thermal parameters were refined using a block-matrix leastsquares procedure. The positional and anisotropic thermal parameters were refined to their final values using a fullmatrix least-squares procedure, minimizing the function $\sum w\left(\left|F_{O}\right|-\left|F_{C}\right|\right)^{2}$ to a conventional residual index of $R=$ $\Sigma\left(\left|\left(\left|F_{O}\right|-\left|F_{C}\right|\right)\right|\right) / \Sigma\left|F_{O}\right|=0.048$ and a weighted residual index 
Table B.12. Positionala $\left(\times 10^{4}\right)$ and Averaged Isotropic Thermal $\left(A^{2}, \times 10^{3}\right)$ Parameters for the Anton in $\left(\mathrm{NHC}_{5} \mathrm{H}_{5}\right)_{10}$

\begin{tabular}{ccccc}
\hline atom & x & $y$ & $z$ & $U_{\text {av }}$ \\
& & & & \\
\hline I1 & 5000 & 5000 & $-635(18) c$ & 77 \\
I2 & 5000 & $1786(8)$ & $4244(11)$ & 43 \\
I3 & $3385(2)$ & $1834(5)$ & $7402(8)$ & 54 \\
I4 & $1887(2)$ & $1854(5)$ & $9636(8)$ & 65 \\
I5 & 5000 & $3525(5)$ & $1407(15)$ & 60 \\
I6 & 0 & $-83(6)$ & $906(12)$ & 48 \\
I7 & 0 & $3313(5)$ & $6146(15)$ & 54 \\
I8 & 0 & $1685(5)$ & $33 \% 1(13)$ & 45 \\
\hline
\end{tabular}

apositional parameters are given as fractions of the unit cell.

$b_{U_{a v}}=\left(U_{11}+U_{22}+U_{33}\right) / 3 . T=\exp \left(-2 \pi^{2}\left(U_{11} h^{2} a^{* 2}+\right.\right.$ $U_{22} k^{2} b^{\star 2}+U_{33} l^{2} c^{\star 2}+2 U_{12} h k a^{*} b^{\star}+2 U_{13} h l a^{\star} c^{\star}+$ $\left.\left.2 U_{23} k 1 b^{\star} c^{\star}\right)\right]$.

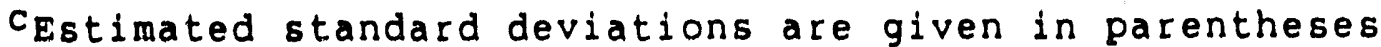
for the least significant digit. 
Table B.13. Anisotropic Thermal Parametersa $\left(\AA^{2}, \times 10^{3}\right)$ for the Anton in $\left(\mathrm{NHC}_{5} \mathrm{H}_{5}\right)_{2} \mathrm{I}_{10}$

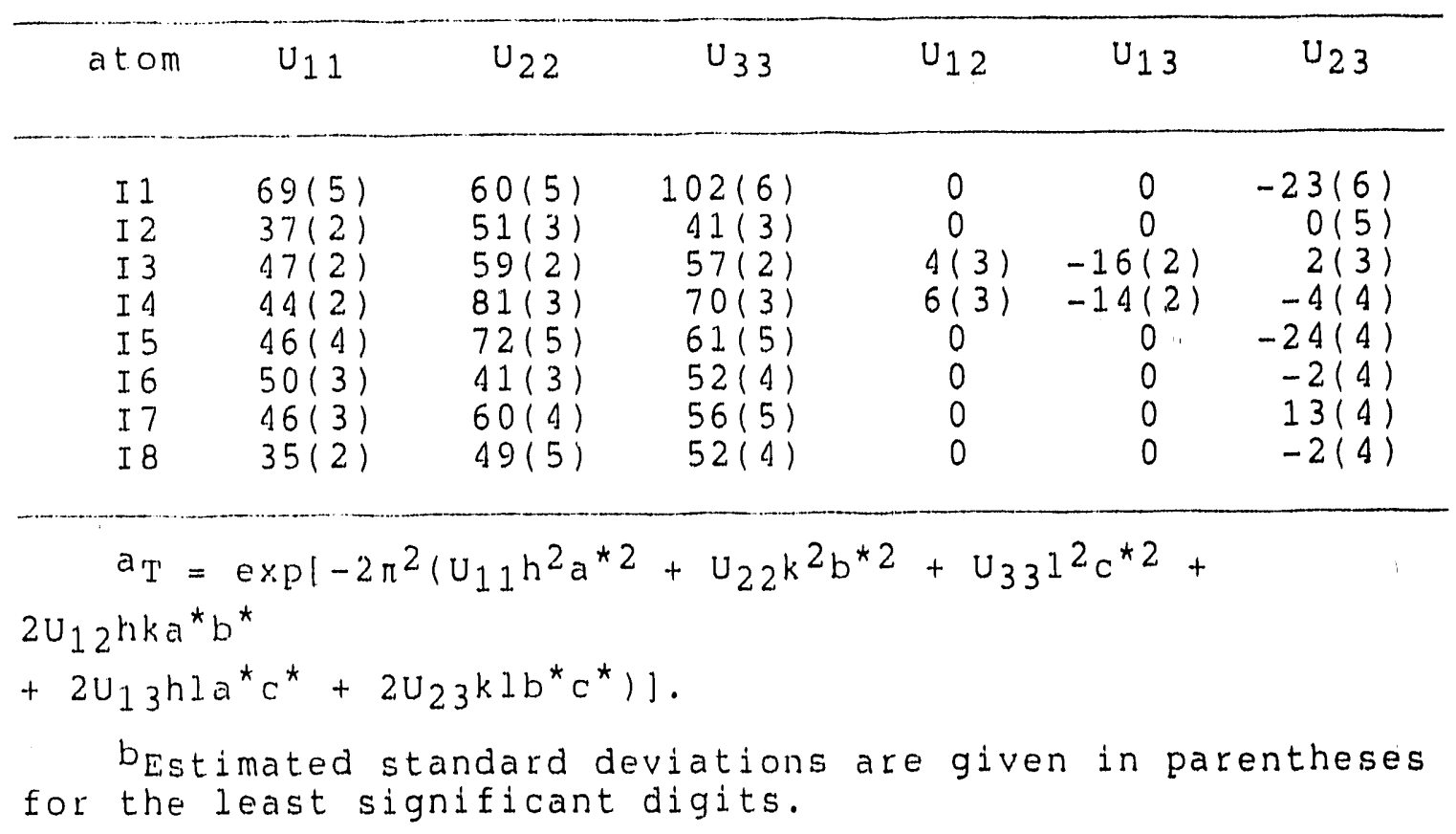


Table B.14. Positionala $\left(\times 10^{4}\right)$ and Isotropic Thermal ( $A^{2}$, $\times 10^{3}$ ) Parameters for the cation in ( $\left.\mathrm{NHC}_{5} \mathrm{H}_{5}\right)_{10}$

\begin{tabular}{llllr}
\hline atom & $x$ & $y$ & $z$ & $U^{b}$ \\
\hline$N$ & $1922(29) c$ & $3915(31)$ & $3014(121)$ & $83(17)$ \\
C1 & $2751(33)$ & $4883(44)$ & $6197(120)$ & $60(16)$ \\
C2 & $3028(31)$ & $4232(43)$ & $5210(209)$ & $90(20)$ \\
C3 & $2623(37)$ & $3627(36)$ & $3437(135)$ & $71(22)$ \\
C4 & $2082(43)$ & $5109(44)$ & $5145(198)$ & $111(26)$ \\
C5 & $1645(27)$ & $4601(30)$ & $3635(123)$ & $55(17)$
\end{tabular}
cell.

apositional parameters are given as fractions of a unit

$b_{T}=\exp \left[-8 \pi^{2} U(\sin \theta / \lambda)^{2}\right]$.

CEstimated standard deviations are given in parentheses for the least significant digits.

Table B.15. Positionala $\left(\times 10^{4}\right)$ and Thermal $\left(A^{2}, \times 10^{3}\right)$ Parameters for Hydrogen Atoms in ( $\left.\mathrm{NHC}_{5} \mathrm{H}_{5}\right)_{10}$

\begin{tabular}{ccccc}
\hline atom & $x$ & $y$ & $z$ & $u^{b}$ \\
\hline H1 & 1589 & 3500 & 1724 & 50.7 \\
H2 & 1063 & 4788 & 2991 & 50.7 \\
H3 & 1864 & 5670 & 5806 & 50.7 \\
H4 & 3085 & 5298 & 7487 & 50.7 \\
H5 & 3610 & 4045 & 5854 & 50.7 \\
H6 & 2841 & 3066 & 2776 & 50.7
\end{tabular}

aHydrogen positions were calculated but not refined. positional parameters are given as fractions of a unit cell. $b_{T}=\exp \left[-8 \pi^{2} U(\sin \theta / \lambda)^{2}\right]$. 
of $\left.R_{W}=\left.\left|\Sigma w\left(\left|F_{0}\right|-\left|F_{C}\right|\right)^{2} / \Sigma w\right| F_{0}\right|^{2}\right)^{1 / 2}=0.048$, wherew $w$ $1 / \sigma^{2}\left(\left|F_{0}\right|\right)$. The positions of the hydrogen atoms were calculated and not refined. The final positional and averaged isotropic thermal parameters for the anion are given in Table B.12, and the anisotrople thermal parameters for these atoms are given in Table B.13. The posttional and isotropic thermal parameters for the cation are given in Table B.14. Table B.15 contains the calculated positions and isotropic thermal parameters for the hydrogen atoms.

Twinning Effects in $\mathrm{YBa}_{2} \mathrm{Cu}_{2} \mathrm{O}_{7-x}$

\section{Crystallographic studies}

Crystals of the superconducting material were prepared by Dr. Gschneidner's group (Ames Laboratory, Iowa state university). Three apparently single-orientation crystals were mounted on glass fibers using Duco cement and attached to standard goniometer heads. The crystals were aligned on a four-circle DATEX diffractometer. Four preliminary $\omega-$ oscillation photographs were taken at various $\phi$ settings. The approximate positions of 10 reflections were selected from the photographs and used as input to an automatic indexing program51. The resulting reduced cell and reduced cell scalars indicated a primitive orthorhombic lattice. Additional reflections having $2 \theta>25^{\circ}$ were used to determine a more accurate orientation matrix. 
A computer program was written to scan a region of reciprocal space using a "step and count" techndque. In this technigue, the detector is moved through readprocal opace using a smalis sopsiae, and the dntensity to masured after each step for several seconds. For each cryatal, regions of reciprocal space around the $(220)$, the $(2 \overline{2} 0)$, the $(400)$ and the $(040)$ reflections were scanned using a stepsize of 0.01 reciprocal lattlce units and a counting time of two seconds. These regions were scanned a minimum of four times and the results of the scans were averaged for each crystal. The plots of the averaged scans for a given reflection were aimilar for each crystal. Representative plots for the $(220),(250),(400)$, and $(040)$ reflections are given in Figures B.5, B.6, B.7, and B.8, respectively. It is quite obvious from the appearance of these plots that the crystalline samples of the material were not singleorientation and that a twinning process was occurring.

\section{Twin Modelling}

A computer program was written to generate the expected shape of a reflection for a given mode of twinning. The effect of instrument broadening was treated by expressing the peak shape as Gaussian,

$$
I_{h}=I_{h_{0}} \exp \left(-\alpha\left(h-h_{0}\right)^{2}\right),
$$




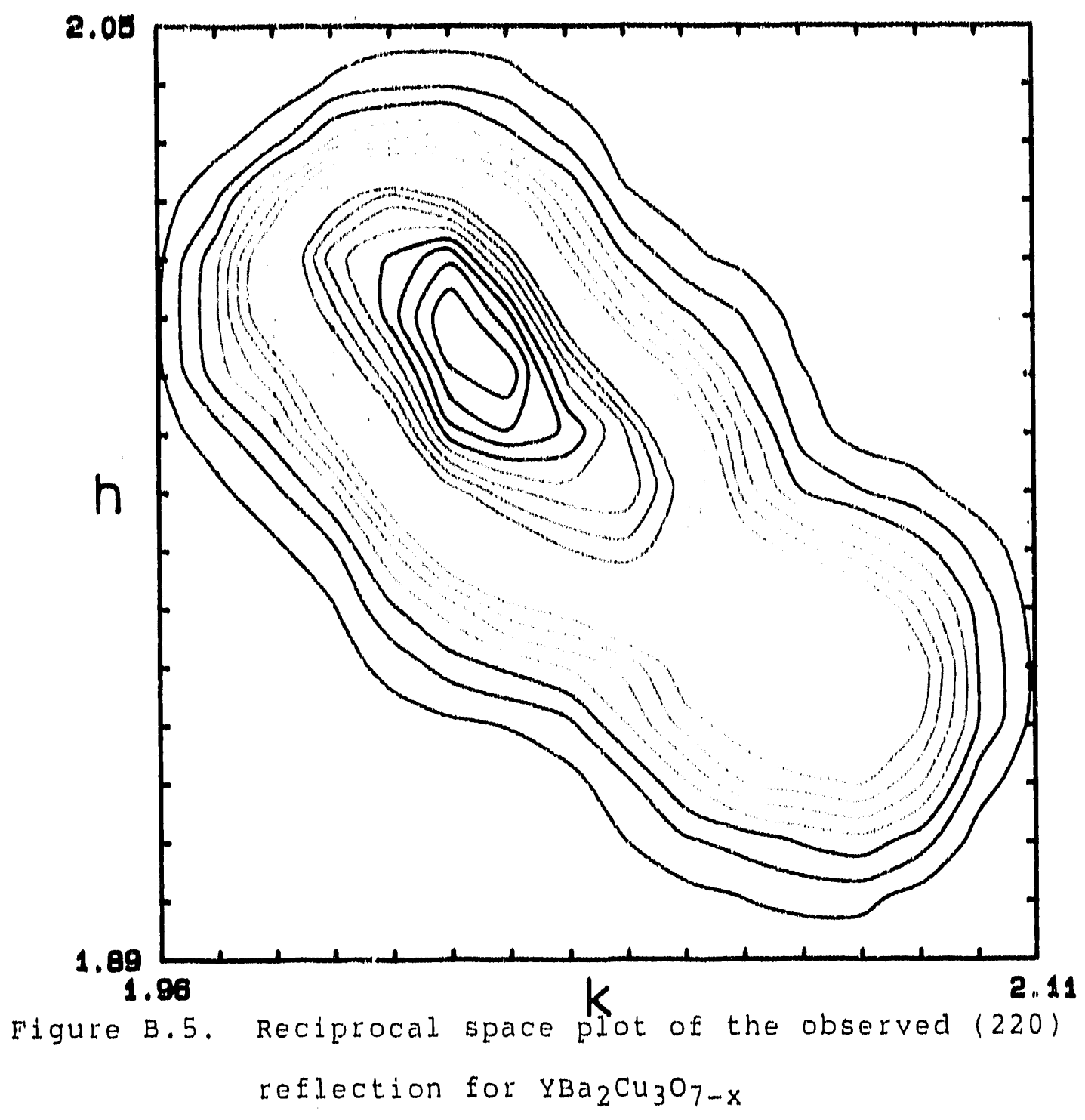




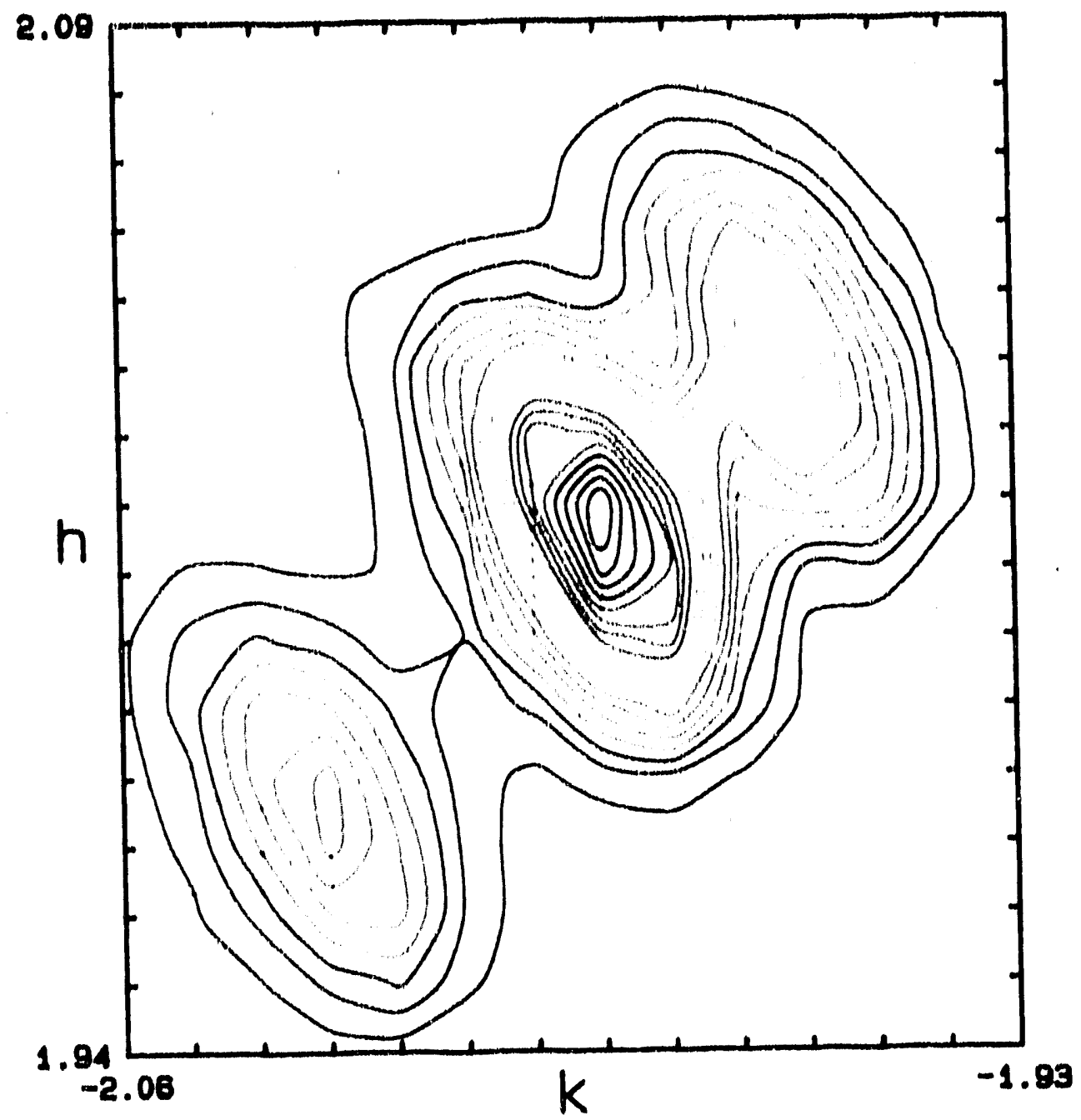

Figure B. 6 . Reciprocal space plot of the observed $(2 \overline{2} 0)$ reflection for $\mathrm{YBa}_{2} \mathrm{Cu}_{3} \mathrm{O}_{7-x}$ 


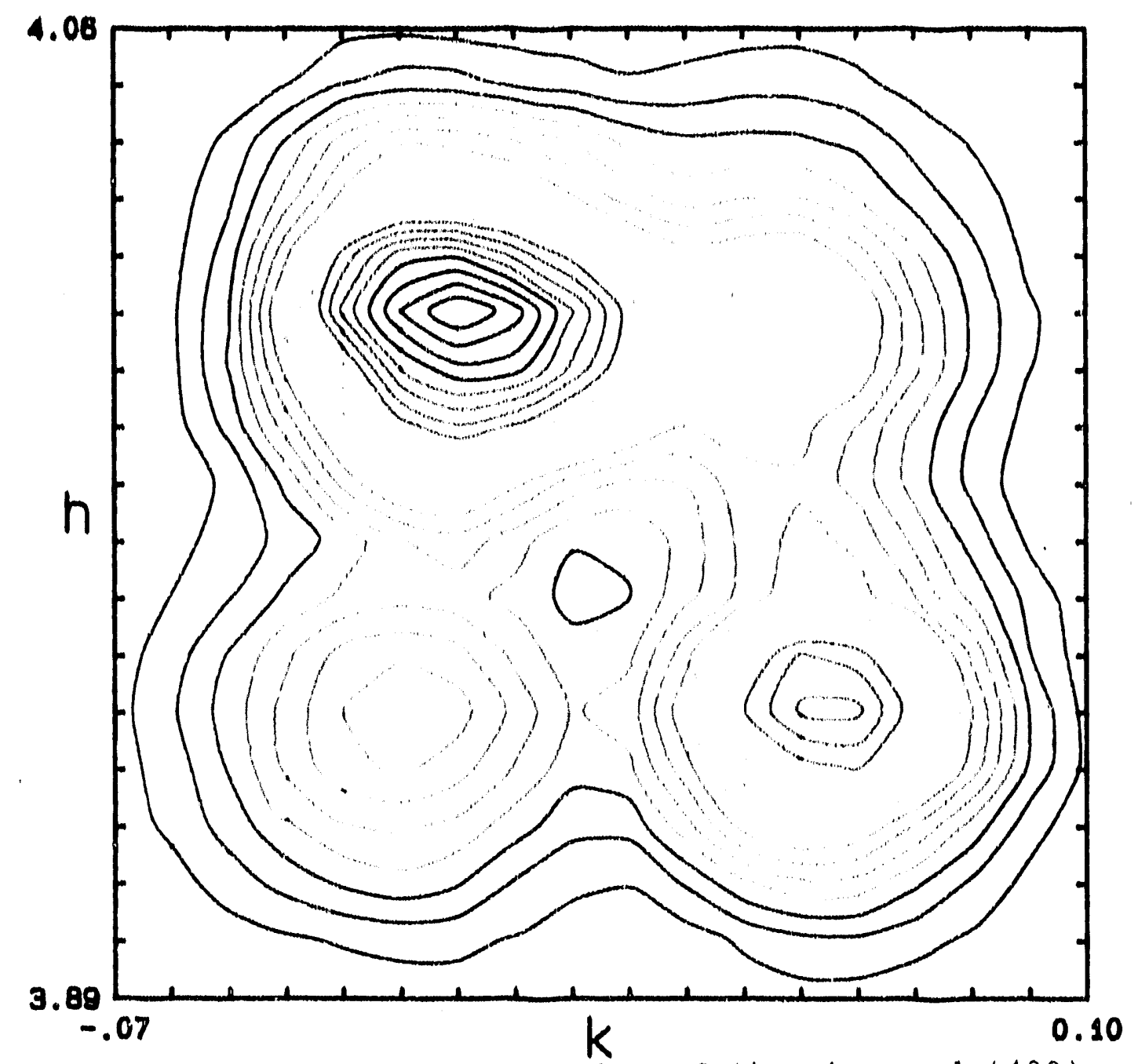

Figure B.7. Reciprocal space plot of the observed (400) reflection for $\mathrm{YBa}_{2} \mathrm{Cu}_{3} \mathrm{O}_{7-x}$ 



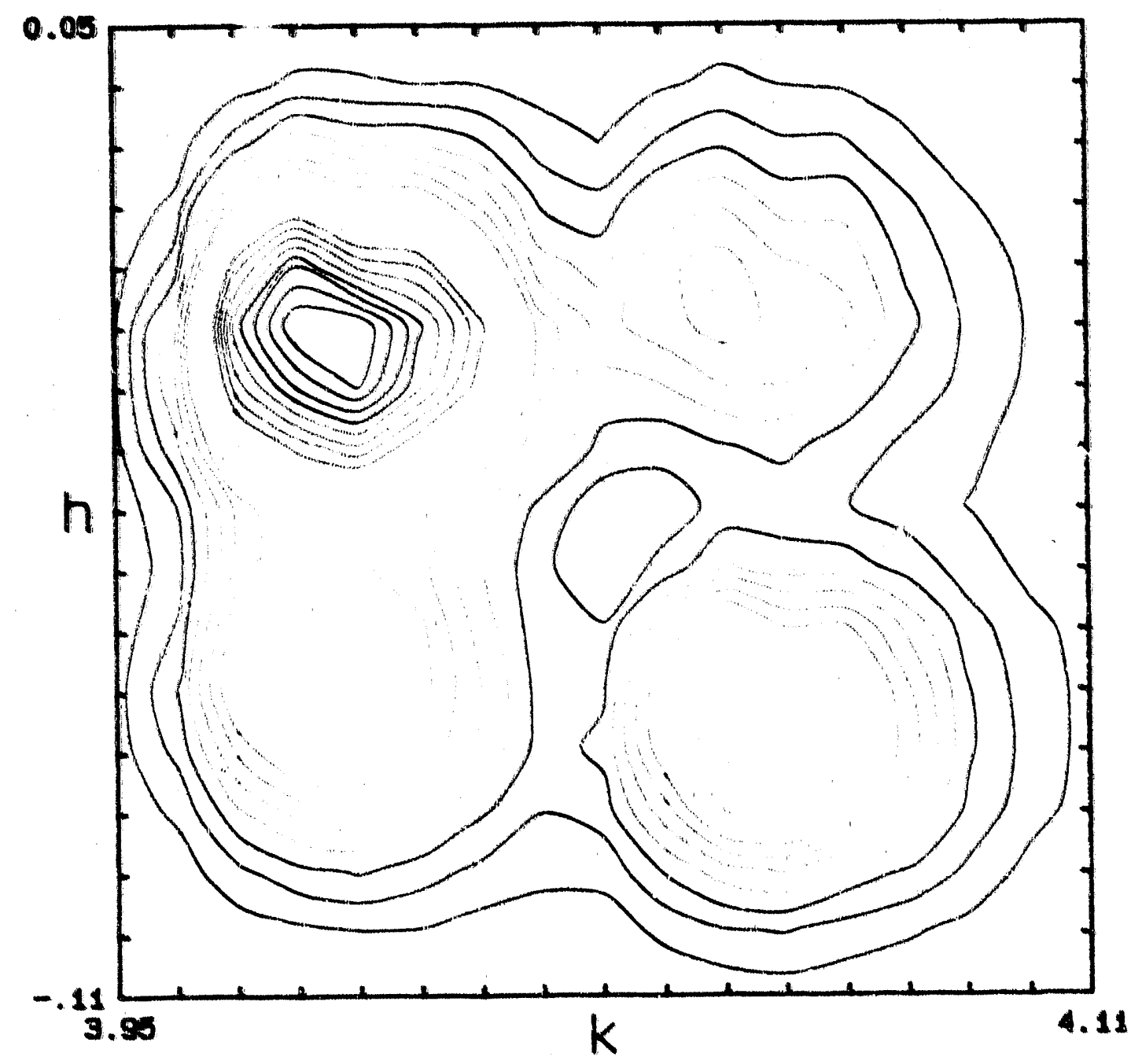

Figure B.8. Reciprocal spice plot of the observed (040) reflection for $\mathrm{YBa}_{2} \mathrm{Cu}_{3} \mathrm{O}_{7-x}$ 
where a is an adjustable parameter which determines the width of the peak. That modes of twinning were modelied: (1) a mircor plane along hr̆o. (2) mirror planes along hho and hño, and (3) a main crystal with a miror alorig hho with a smalle satelite. also having mircor along hro, rotated 90 relative to the orientation of the main crystal. plots of the (400), the (220), and the (220) reflections for each of the twinning modes are shown in Figures B.9 through B.1T.

\section{Discussion}

At high temperatures, this compound exists in a tetragonal form, having mircos along hho and hio and a fout-fold cotation axis. As it cools, it undergos a transformation to an orthorhombio phase. The accepted space group for this phese is pmm. This space group contains miscol planes along each axis, so that a twinning mode containing miror hĭo must, by symetry, have a miror along hho. In such a twinning mode, the $(220)$ and the $(220)$ reflectons should have the same shape. However, examination of the observed rellections clearly indicates that this is not the case. The (400) reflection should have thee majo: components, as shown in Flquee 5.10, but again. this is not the case.

The chinning model that gives the best agleement to the observed peak shapes has a main cystal with a mirror along 


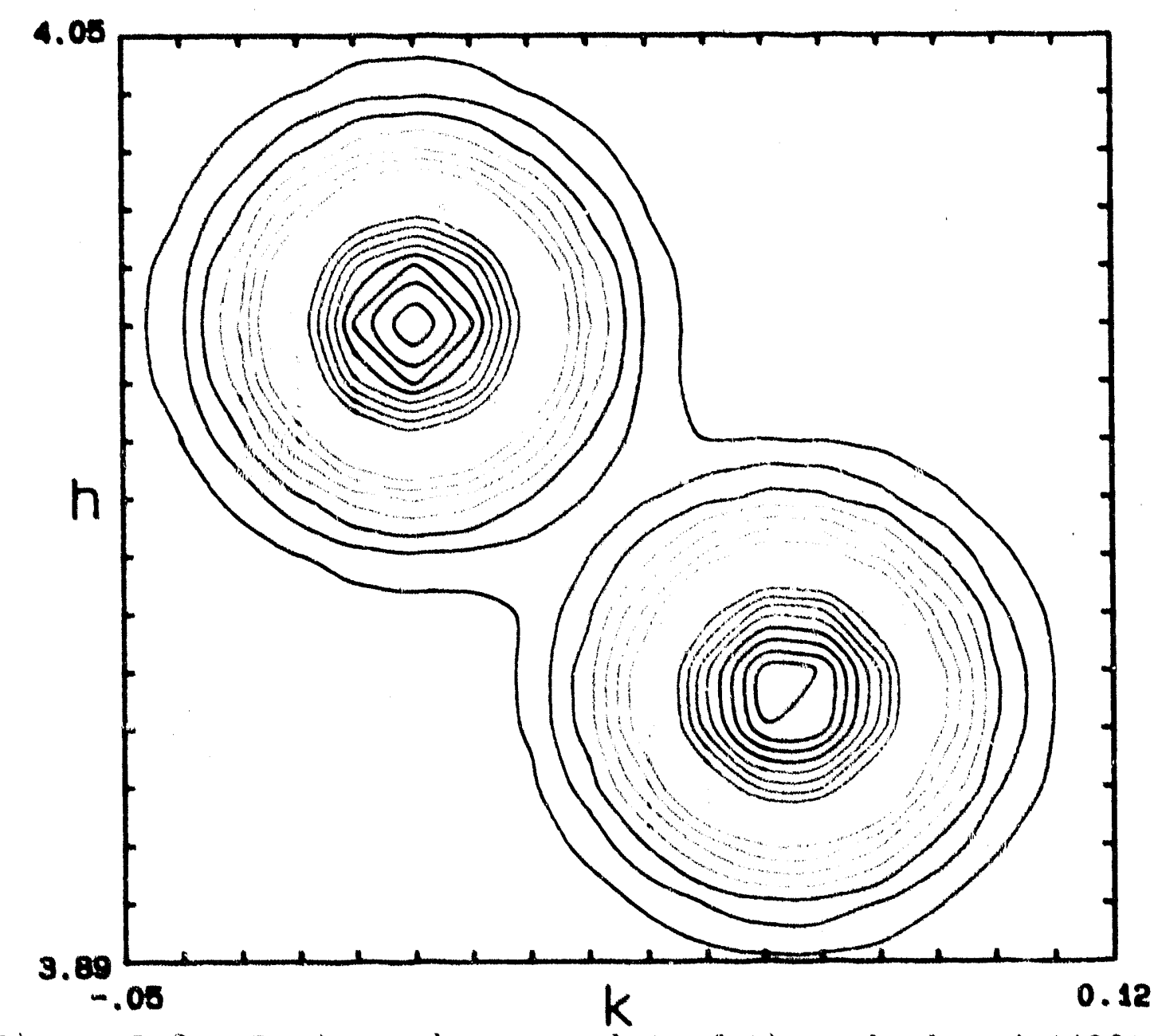

Figure B.9. Reciprocal space plot of the calculated (400) reflection for $\mathrm{YBa}_{2} \mathrm{Cu}_{3} \mathrm{O}_{7-x}$ using twinning mode (1) 


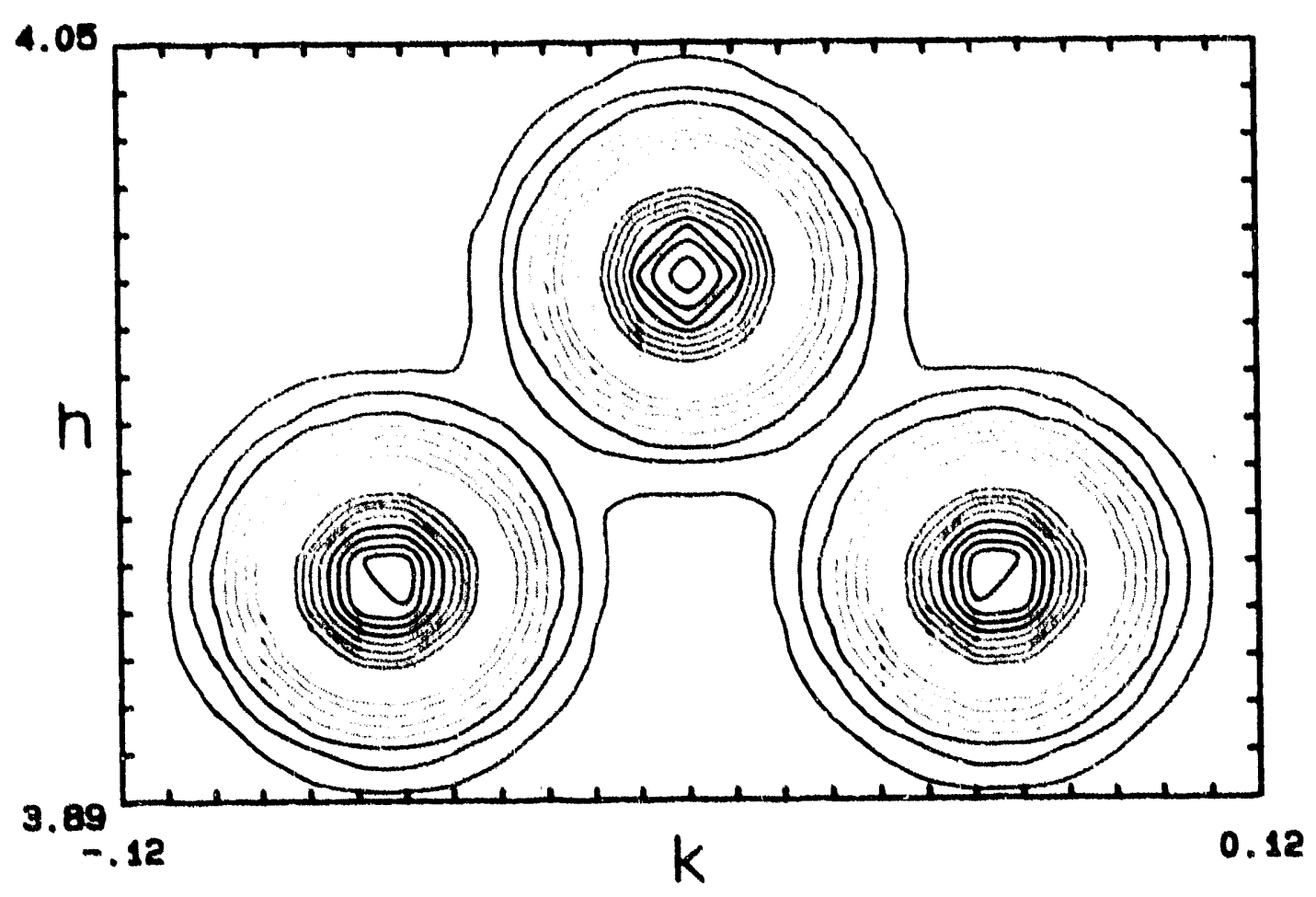

Figure B.10. Reciprocal space plot of the calculated (400) reflection for YBa $\mathrm{Cu}_{3} \mathrm{O}_{7}-\mathrm{x}$ using twinning mode (2) 


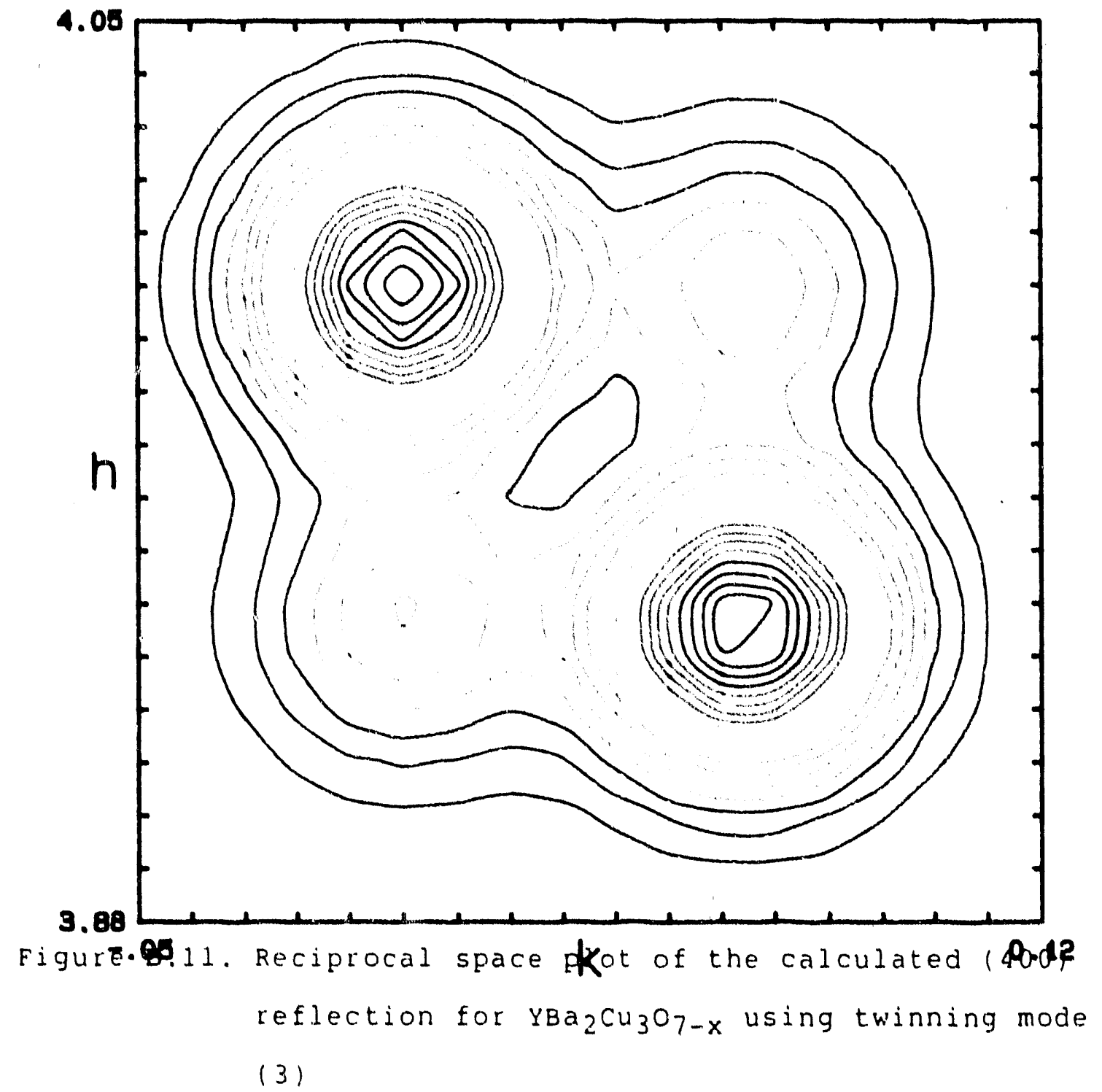

(3) 


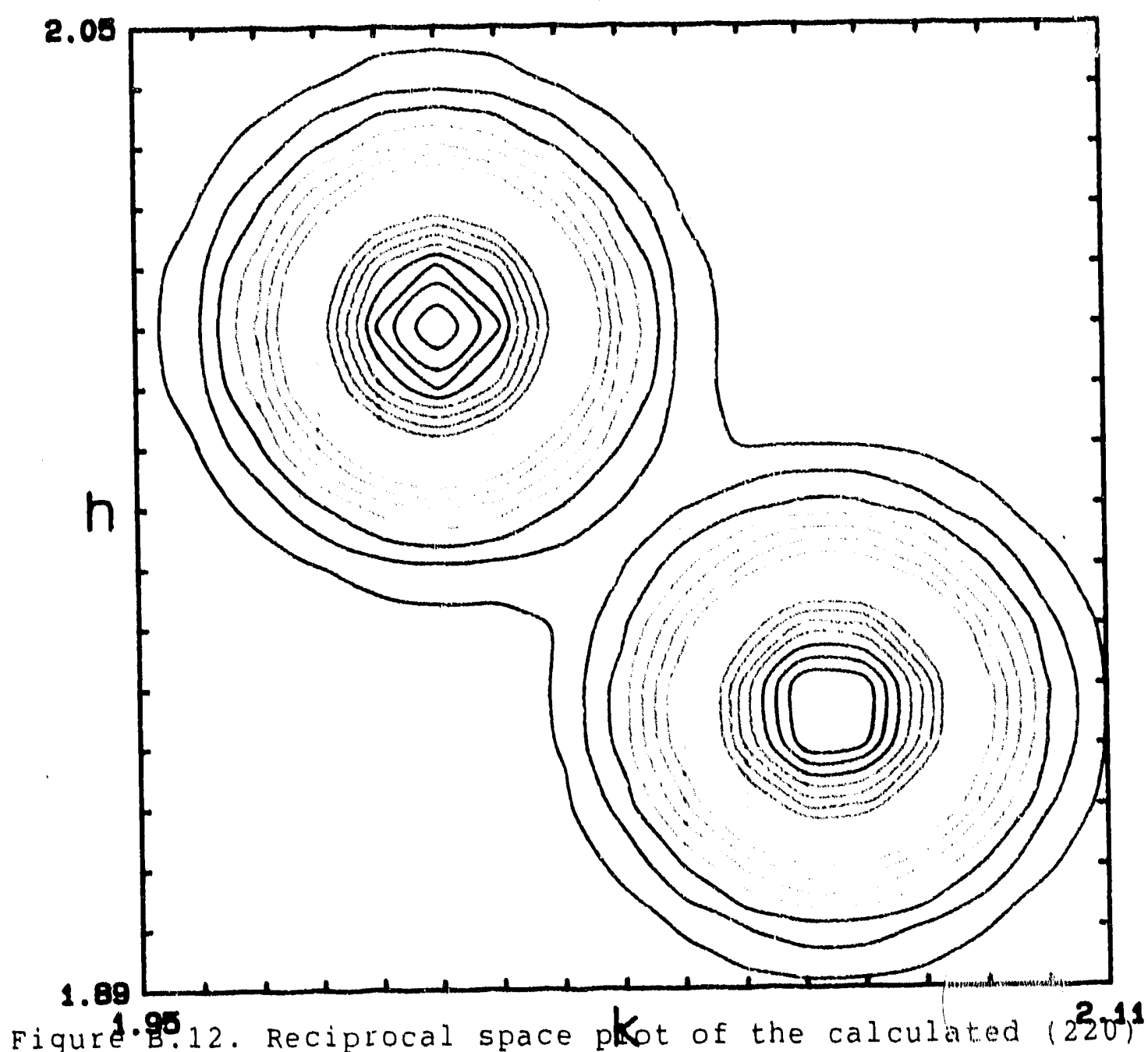

reflection for $\mathrm{YBa}_{2} \mathrm{Cu}_{3} \mathrm{O}_{7-x}$ using twinning mode (1) 


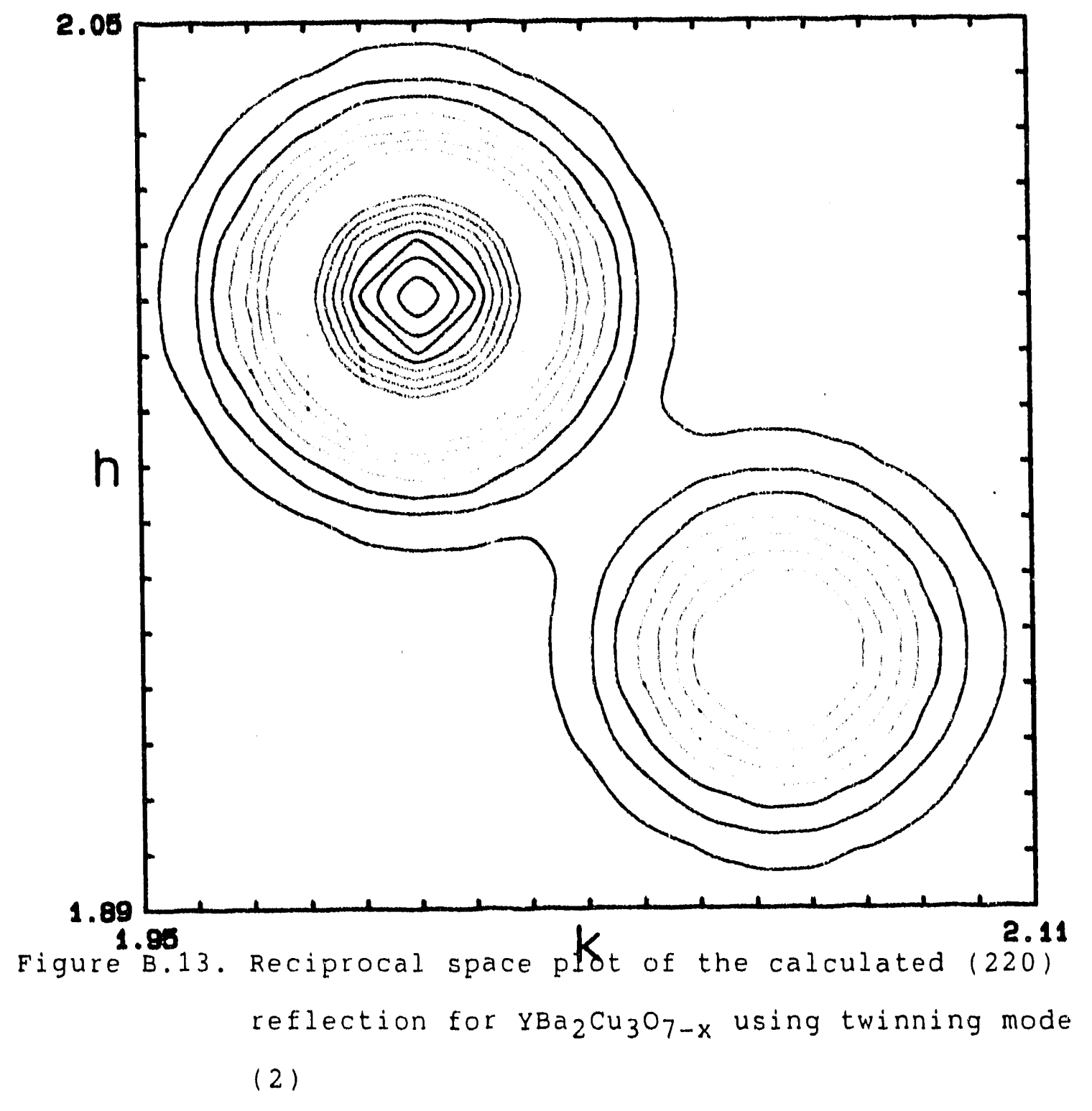




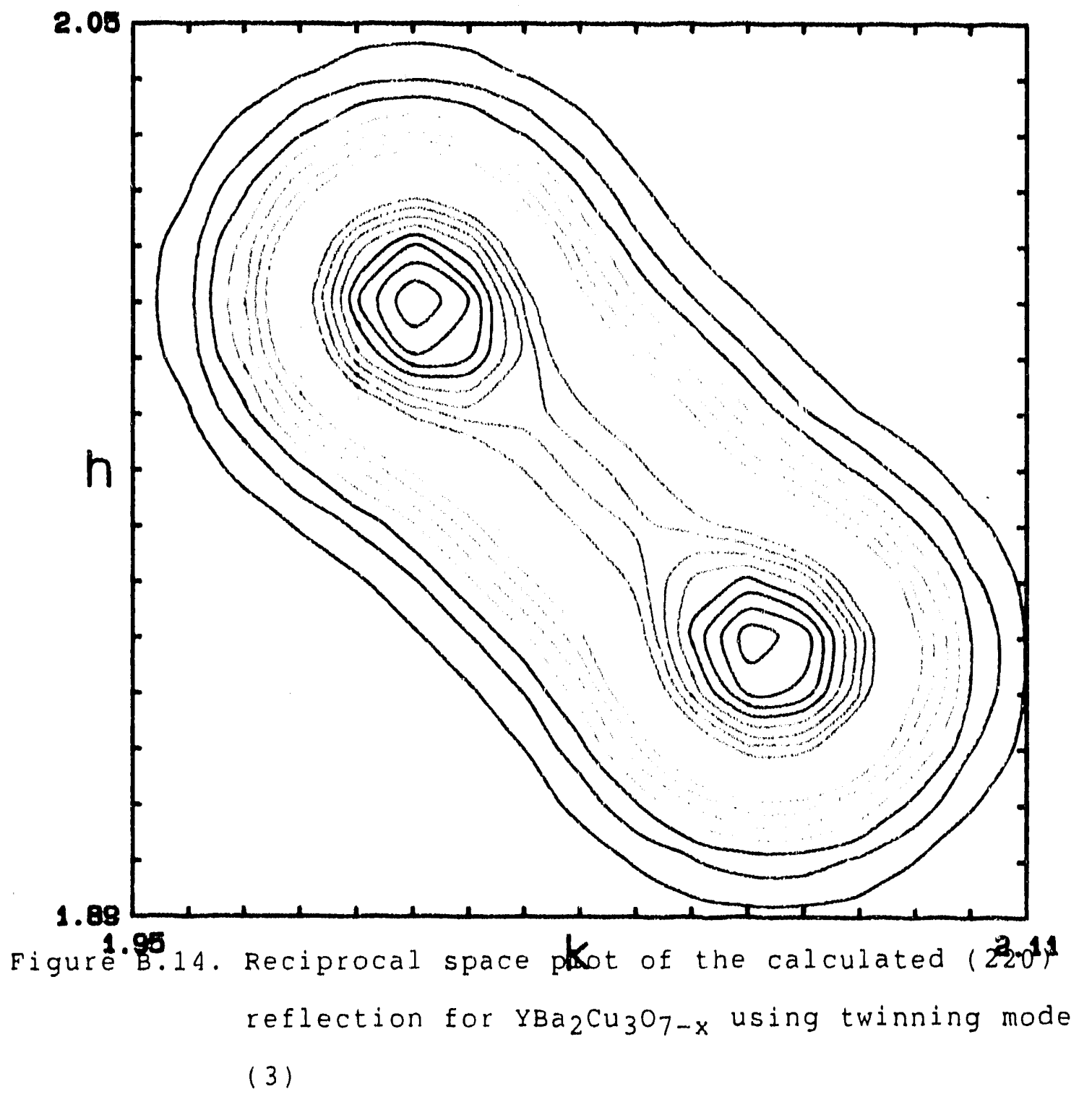




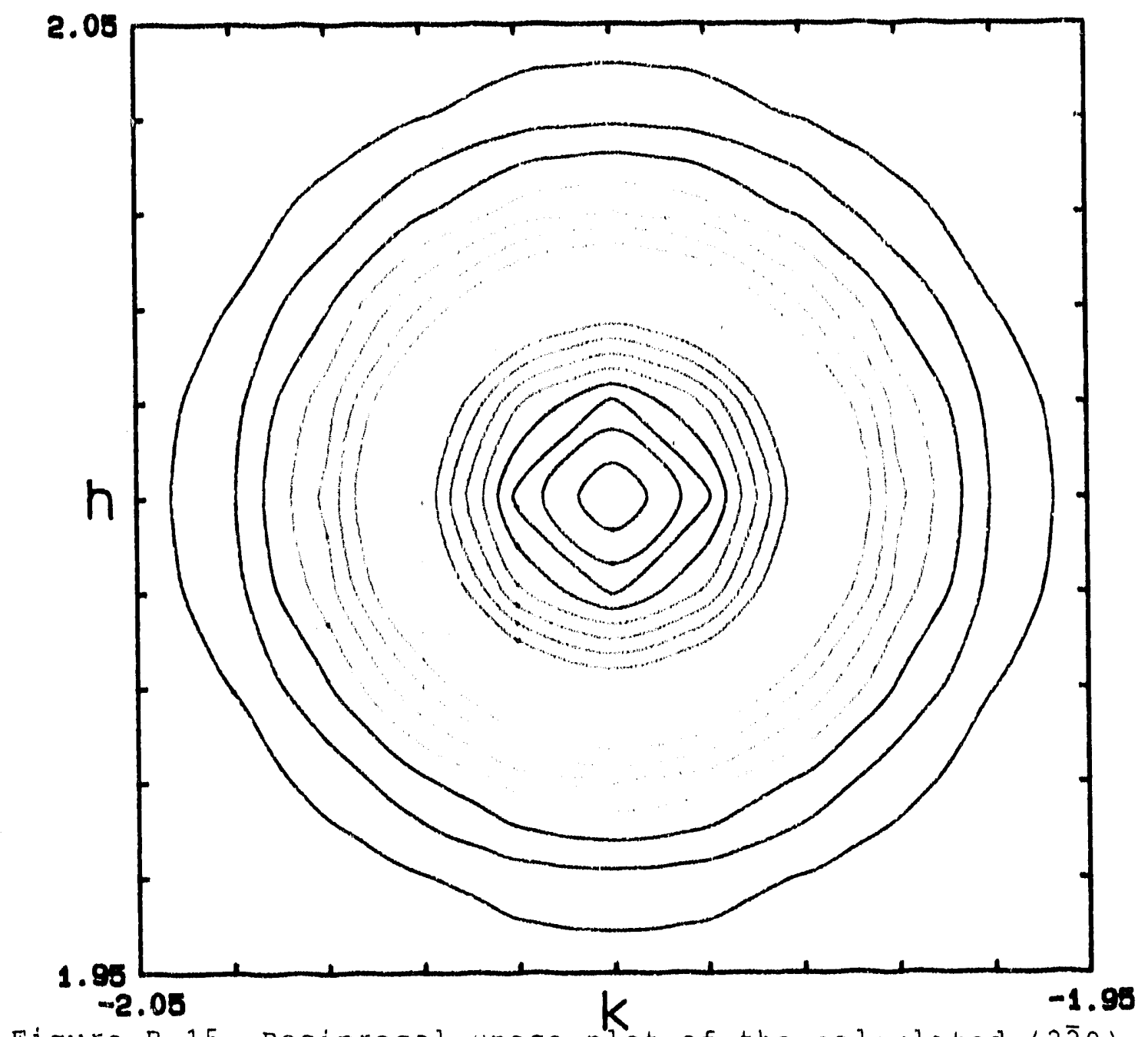

Figure B.15. Reciprocal pace plot of the calculated $(2 \overline{2} 0)$ reflection for $\mathrm{YBa}_{2} \mathrm{Cu}_{3} \mathrm{O}_{7-x}$ using twinning mode (1) 


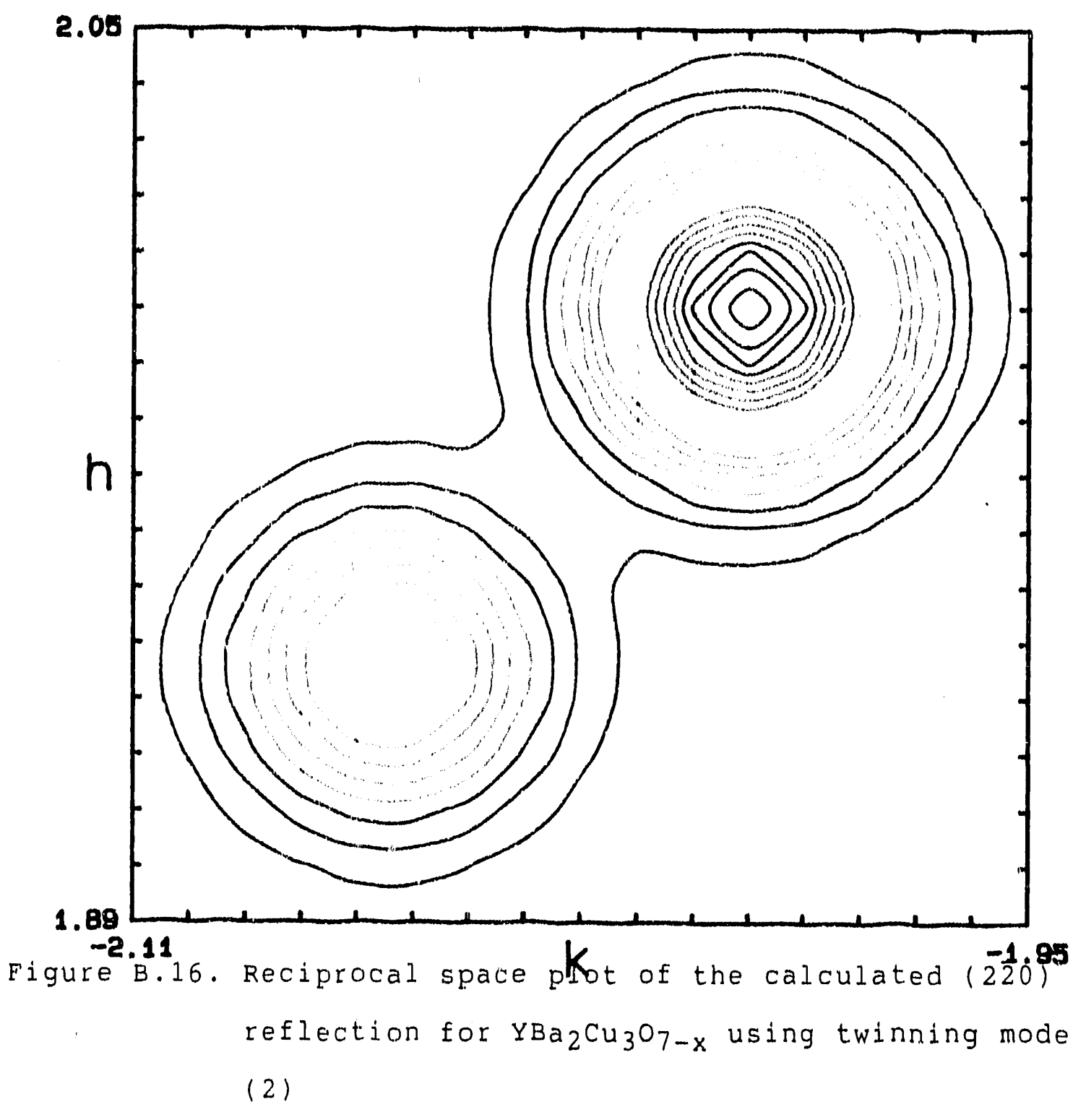




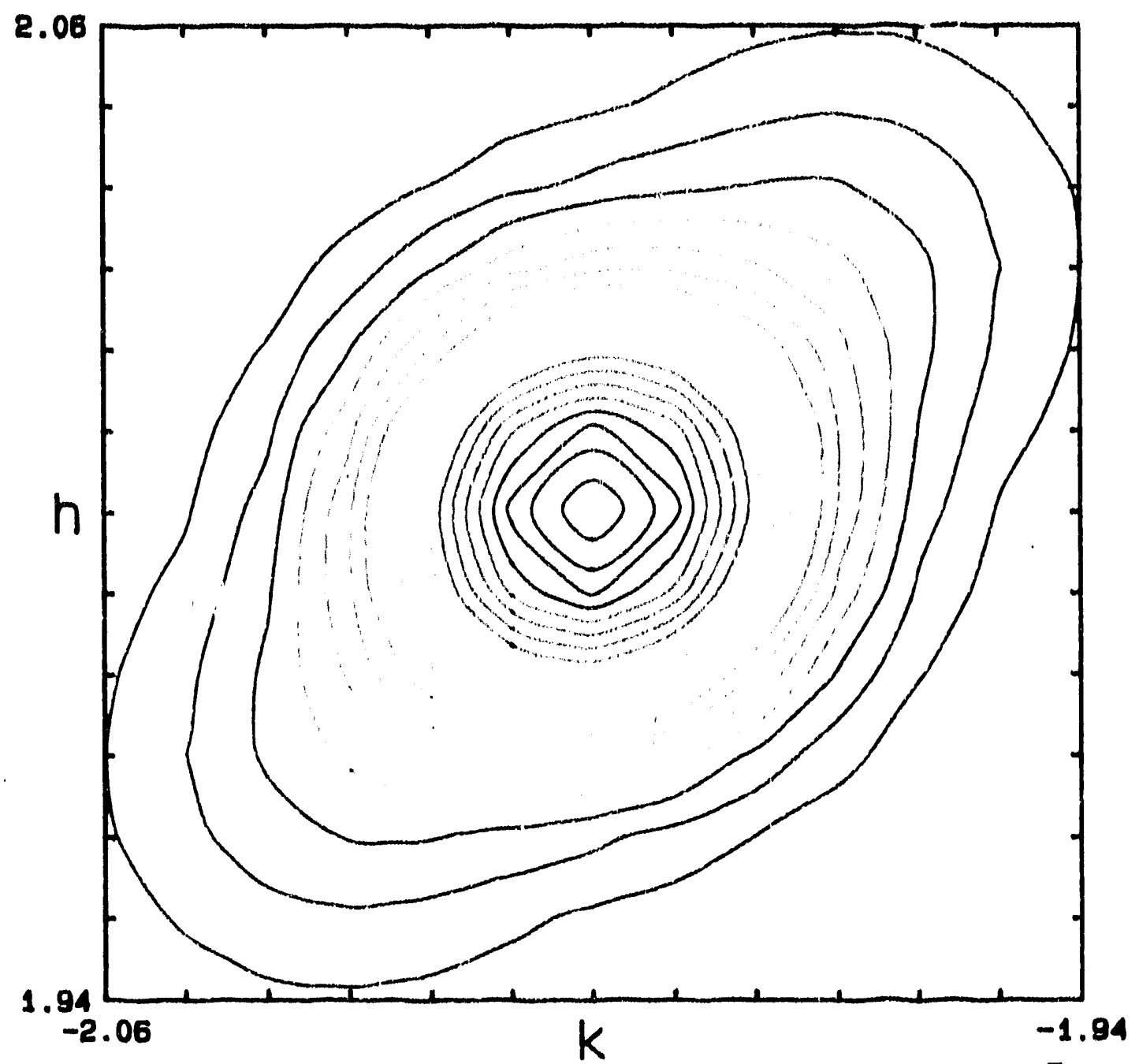

Figure B.17. Reciprocal space plot of the calculated $(2 \overline{2} 0)$ reflection for $\mathrm{YBa}_{2} \mathrm{Cu}_{3} \mathrm{O}_{7-x}$ using twinning mode (3) 
hFio and a satelitte rotated by $90^{\circ}$. It is not unexpected that a rotated satelilte is found, as this could easily be the result of the loss of the four-fold rotation axis. It is surprising that the model only contains one mirror. This may indicate that the correst space group is of lower orthorhombic symmetry, or that there is some type of longrange "memory" effect occurring so that once twinning begins to occur in one direction, that direction becomes the preferted twinning direction. 
1. W. L. Bragg, Proc. Cambridge Phil. Soc., 17, 43 (1.913).

2. D. Harker and J. S. Kasper, Asta Crystallogr, 1,70 (1948).

3. J. Karle and H. Hauptman, Acta Crystallogr, 3, 181 (1950).

4. D. Sayle, Acta Crystallogr., 5, 60 (1952).

5. M. Cochran, Acta Crystallogr., 5, 65 (1952).

6. W. H. Zachariasen, Acta Crystallogr., 5, 68 (1952).

7. W. Cochran and M. M. Woolfson, Acta Crystallogr., 1 (1955).

8. W. Cochran, Acta Crystallogr., 8, 473 (1955).

9. J. Karle and H. Hauptman, Acta Crystallogr., 9, 365 $(1956)$.

10. I. L. Karle and J. Karle, Acta Crystallogr., 16, 969 $(1964)$.

11. G. Germain, P. Main and M. M. Woolfson, Acta Crystallogr., B26, $274(1970)$.

12. D. White and M. M. Woolfson, Acta Crystallogr,, A31, 53 (1975).

13. H. Hauptman, Acta Crystallogr., A31, 671 (1975).

14. H. Hauptman, Acta Crystallogr,, A31, 680 (1975).

15. H. Schenk, Acta Crystallogr., A29, 480 (1973).

16. H. Schenk, Acta Crystallogr., A30, 477 (1974).

17. H. Schenk and J. G. H. de Jong, Acta Crystallogr., A. 2 , $31(1973)$.

18. Yao Jia-xing, Acta Crystallogr., A37, 642 (1981).

19. R. Narayan and R. Nityananda, Curr. Sci., 50, 168 (1981). 
20. R. Narayan and R. N1tyananda, Acta Cryeta110gr., A38, $122(1982)$.

21. G. Bricogne, Acta Crystallogr, A40, 410 (1984).

22. A. L. Patterton, Phys. Rev., 46, 372 (1934).

23. J. Richardson, Jr., Ph.D. dissertation, Iowa state Univelsity, Ames, Iowa, 1984.

24. D. Harker, J. Chem. Phys, 4, 381 (1936).

25. A. Mighi11, Ph.D. dissertation, Princeton University, Princeton, New Jersey, 1963.

26. J. Clastre and R. Gay, Compt. Rend., 230, 1876 (1950).

27. J. Garido, Compt. Rend., 230, 1878 (1950).

28. M. Buerger, "Vector Space", Wiley: New York, 1959.

29. R. A. Jacobson, Trans. Am. Crystallogr. Assoc., 2 (1966).

30. D. W. Green, V. N. Ingram and M. F. Perutz, Proc. Roy SOC., $\mathrm{A225}, 287(1954)$.

31. P. Tollin and M. G. Rossman, Acta Crystallogr., 21, 872 (1966).

32. C. E. Nordman, Trans. Am. Crystallogr. Assoc, 2 2, 29 (1966).

33. G. M. Sheldrick, in "Crystallographic Computing 3 ", edited by G. M. Sheldrick, C. Kruger and R. Goddard, p. 175, Oxford University Press, New York, 1985.

34. F. Pavelcik, J. Appl. Crystallogr., 19, 488 (1986).

35. P. Luger and J. Fuchs, Acta Crystallogr., A42, 380 (1966).

36. T. C. Terwilliger, S. - H. Kim and D. Eisenberg, Acta Crystallogr, A43, $1(1.987)$.

37. S.-L. Wang, Ph.D. dissertation, Iowa state University, Ames, Iowa, 1985.

38. Allen, P. W. and sutton, L. E., Acta Crystallogr., 3, $46(1950)$. 
39. Defaver, P. W. and Jacobson, R. A., Cryst. Struct. Commun., 5, 31 i $(1976)$.

40." Hackert, M. L., Jacobson, R. A., and Keiderling, T. A., Inorg. Chem., 10, 1075 (1971).

41. Hubbard, C. R. and Jacobson, R. A., Inorg. Chem., 11, $2247(1972)$.

42. Lawton, S. I. and Jacobson, R. A., Inorg. Chem., $743(1966)$.

43. Lamton, S. I. and Jacobson, R. A., Inorg. Chem., I. $2124(1968)$.

44. Lanton, S. I. and Jacobson, R. A., Inorg. Chem., 10, 709 (11971).

45. Lamton, S. L. and Jacobson, R. A., and Frye, R. S., Inorg. Chem., 10, 701 (1971)

46. Porter, S. K. and Jacobson, R. A., I. Chem. Soc. (A). 1356 (1970).

47. Porter, S. R. and Jacobson, R.A., J. Chem. Soc. (A). 1359 (1970).

48. Porter, S. K. and Jacobson, R. A., Cryst. Struct. Cominum. I, 431 (1971).

49. Schroeder, D. R. and Jacobson, R. A., Inorg. Chem.. 12. $515(1972)$.

50. Wismer, $\mathbb{R}$. $K$. and Jacobson, $\pi$. A., Inorg. Chem, 13, $1678(1974)$.

51. Jacobson, R. A., J.Appl. Crystallogr., 9, 115 $(1976)$.

52. Lapp. R. L. and Jacobson, R. A., MLLS: A Generalized Crystaliographic Least-Squares Program", USDOE Report IS-4708; IOnia state University, Ames IA, 1978.

53. Pontil, R. R. and Jacobson, R. A., "FoUR: A Generailized Crystallographic Fourier Program , USDOE Report IS-4737; IDá state University. Ames IA, 1980 .

54. Zachariasen, W. H., J. Less Common Metals, 62, 1 (1978). 


\section{ACKNOWLEDGEMENTS}

I wish to thank Dr. Robert Jacobson for his guidance and encouragement during the course of this work. I will take with me a very vivid image of him standing at the chalkboard, drawing pictures of unit cells and "solving" the phase problem. Many of the concepts in this approach came from those chalkboards.

I would like to thank our secretary, Brenda Smith, for all of her help and her friendship. I'm sure that she must have thought that $I$ was being very antisocial my first year in the group.

I would also like to thank former and present group members Lance Miller, Raman, Dave Wintergrass, Ying-zhong Su, and Cathy Day for their help in learning crystallography, their interest in my research and their willingness to be guinea pigs when I modified a procram.

During my stay in Ames, I have had the privilege of meting some very fine people. I think that I shall always remember the sunday evening barbecues with Ross and Janet Nord, David and Diane Sanders, and Jeff Crain. I will certainly never forget the "turkey wine" in Boone and the infamous trip to Pittsburg for pizza. I am also grateful for the friendships that I have had with fellow graybeard Clark Carleson, Deb Spink, Dick Nagaki and Susan Zvacek. 
All of them have made Ames a much more enjoyable place to live. Elen sila lúmenn omentilmo.

And finally, I would like to thank my parents and family for their support. They have stood behind me throughout the course of my graduate studies.

This work was performed under contract No. W-7405-Eng-82 with the U. S. Department of Energy and this thesis has been assigned a report number of IS-T-1408. 

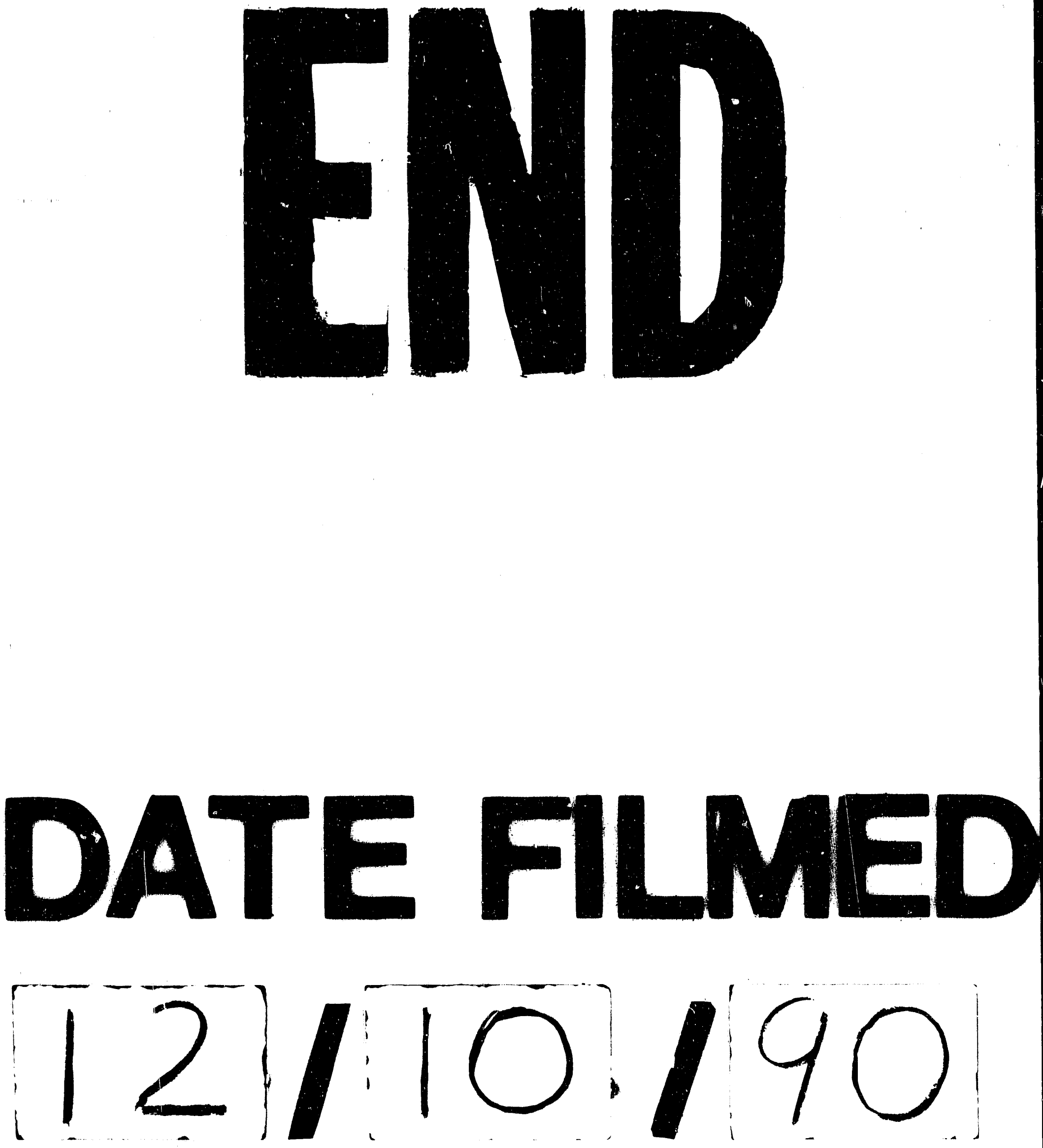
